\title{
Functionalization of 1,3,4-Oxadiazoles and 1,2,4-Triazoles via Selective Zincation or Magnesiation using TMP-bases
}

Kuno Schwärzer, ${ }^{\dagger}$ Carl Phillip Tüllmann, ${ }^{\dagger}$ Simon Graßl, ${ }^{\dagger}$ Bartosz Górski, ${ }^{\dagger}$ Cara Brocklehurst, ${ }^{\ddagger}$ and Paul Knochel ${ }^{*, \dagger}$

\author{
${ }^{\dagger}$ Department of Chemistry \\ Ludwig-Maximilian-Universität \\ Butenandtstraße 5-13 \\ 81377 München, Germany
}

¥Global Discovery Chemistry

Novartis Institutes for BioMedical Research

141 Klybeckstraße

4057 Basel, Switzerland

\section{Supporting Information}




\section{General information}

All reactions were carried out under an argon atmosphere in flame-dried glassware. Syringes which were used to transfer anhydrous solvents or reagents were purged with argon prior to use. Yields refer to isolated yields of compounds estimated to be $>95 \%$ pure as determined by ${ }^{1} \mathrm{H}-\mathrm{NMR}\left(25^{\circ} \mathrm{C}\right)$ and capillary GC. THF was continuously refluxed and freshly distilled from sodium benzophenone ketyl under nitrogen and stored under argon over molecular sieves. Solvents for column chromatography were distilled prior to use. All reagents were obtained from commercial sources and used without further purification unless otherwise stated. Reactions were cooled using acetone / dry ice baths and heated using an oil bath on a magnetic stirrer.

\section{Reagents}

$n$ BuLi solutions in hexane were purchased from Albemarle or Sigma Aldrich and the concentration was determined by titration against $\mathrm{N}$-benzylbenzamide in THF at $-20{ }^{\circ} \mathrm{C}$ or $0{ }^{\circ} \mathrm{C}$ respectively. ${ }^{1}$

TMPH was purchased from Albemarle (Frankfurt, Germany), freshly distilled over $\mathrm{CaH}_{2}$ and stored under argon.

CuCN.2LiCl solution ( $1.00 \mathrm{M}$ in THF) was prepared by drying CuCN $(8.96 \mathrm{~g}, 100 \mathrm{mmol}$, 1.00 equiv) and $\mathrm{LiCl}$ (8.48 g, $200 \mathrm{mmol}, 2.00$ equiv) in a Schlenk-flask under vacuum for $5 \mathrm{~h}$ at $150{ }^{\circ} \mathrm{C}$. After cooling to $25^{\circ} \mathrm{C}$, dry $\operatorname{THF}(100 \mathrm{~mL})$ was added and stirred until the salts were dissolved.

$\mathrm{ZnCl}_{2}$ solution (1.00 M in THF) was prepared by drying $\mathrm{ZnCl}_{2}(27.3 \mathrm{~g}, 200 \mathrm{mmol})$ in a Schlenkflask under vacuum for $5 \mathrm{~h}$ at $150{ }^{\circ} \mathrm{C}$. After cooling to $25^{\circ} \mathrm{C}$, dry THF (200 mL) was added and stirred until the salts were dissolved.

iPrMgCl. $\mathrm{LiCl}$ in THF was prepared by flame drying magnesium turnings $(24 \mathrm{~g}, 1.0 \mathrm{~mol}, 2.0$ equiv) and anhydrous $\mathrm{LiCl}$ ( $25 \mathrm{~g}, 0.60 \mathrm{~mol}, 1.2$ equiv) in a Schlenk-flask under vacuum at 450 ${ }^{\circ} \mathrm{C}$. After the addition of anhydrous THF (500 mL), $\mathrm{PrCl}(39 \mathrm{~g}, 0.50 \mathrm{~mol}, 1.0$ equiv) was added dropwise at r.t. using a dropping funnel until the reaction started. Then the reaction mixture was cooled to $0{ }^{\circ} \mathrm{C}$ and the addition was continued overnight while allowing it to warm up to r.t.. The remaining solids were filtered off and the $\mathrm{iPrMgCl} \cdot \mathrm{LiCl}$ was titrated against iodine.

\footnotetext{
${ }^{1}$ Burchat, A. F.; Chong, J. M.; Nielsen, N. J. Organomet. Chem. 1997, 542, 281.
} 
The hydroxyamino benzoates were prepared according to a literature procedure. ${ }^{2}$

\section{Chromatography}

Flash column chromatography (FCC) was performed using $\mathrm{SiO}_{2} 60(0.040-0.063 \mathrm{~mm}, 230$ 400 mesh ASTM) from Merck. Thin layer chromatography (TLC) was performed using aluminum plates covered with $\mathrm{SiO}_{2}$ (Merck 60, F-254). Spots were visualized by UV light irradiation and/or by staining of the TLC plate with one of the reagents below, followed by heating with a heat gun if necessary.

- $\mathrm{KMnO}_{4}(0.3 \mathrm{~g}), \mathrm{K}_{2} \mathrm{CO}_{3}(20 \mathrm{~g})$ and $\mathrm{KOH}(0.3 \mathrm{~g})$ in water $(300 \mathrm{~mL})$.

- $\mathrm{Ce}\left(\mathrm{SO}_{4}\right)_{2}(5.0 \mathrm{~g}),\left(\mathrm{NH}_{4}\right)_{6} \mathrm{Mo}_{7} \mathrm{O}_{24} \cdot 4 \mathrm{H}_{2} \mathrm{O}(25 \mathrm{~g})$ and conc. $\mathrm{H}_{2} \mathrm{SO}_{4}(50 \mathrm{~mL})$ in water (450 mL).

- Neat iodine absorbed on silica gel.

\section{Preparative HPLC}

For purification, an Agilent Technologies 1260 Infinity HPLC-System was used, consisting of two prep-pumps (acetonitrile/water, no additives), a MWD-detector (210 nm wavelength, $40 \mathrm{~nm}$ bandwidth, ref-wavelength $400 \mathrm{~nm}$, ref-bandwidth $100 \mathrm{~nm}$ ) and a fraction collector. Three different columns were used: 1) Kinetix EVO C18 $5 \mu \mathrm{m}$ column (length: $150 \mathrm{~mm}$, diameter: $10 \mathrm{~mm}$ ), 2) Kinetix EVO C18 $5 \mu \mathrm{m}$ column (length: $150 \mathrm{~mm}$, diameter: $21.2 \mathrm{~mm}$ ) and 3) Waters XBridge Prep C8 5 um column (length: $150 \mathrm{~mm}$, diameter: $30 \mathrm{~mm}$ ).

\section{Analytical data}

NMR spectra were recorded on Bruker ARX 200, AC 300, WH 400or AMX 600 instruments. Chemical shifts are reported as $\delta$-values in ppm relative to the deuterated solvent peak: $\mathrm{CDCl}_{3}$ $\left(\delta_{H}: 7.26 ; \delta_{C}: 77.16\right)$. For the observation of the observed signal multiplicities, the following abbreviations and combinations thereof were used: $s$ (singlet), $d$ (doublet), $t$ (triplet), q (quartet), quint (quintet), sext (sextet), sept (septet), m (multiplet) and br (broad). If not otherwise noted, the coupling constants given are either $\mathrm{H}-\mathrm{H}$ or $\mathrm{H}-\mathrm{F}$ coupling constants for proton signals and C-F coupling constants for carbon signals. In the cases where a steric

\footnotetext{
2 a) Graßl, S.; Chen, Y.-H.; Hamze, C.; Tüllmann, C. P.; Knochel, P. Org. Lett. 2019, 212, 494. b) Chen, Y.H.; Graßl, S.; Knochel, P. Angew. Chem. Int. Ed. 2018, 57, 1108; Angew. Chem. 2018, 130, 1120.
} 
centre is located next to a bicyclopentane core, the protons of the methylene groups in the BCP unit are diastereotopic and show the splitting pattern expected for the resulting $[\mathrm{AB}]_{3}$-system.

Melting points are uncorrected and were measured on a Büchi B.540 apparatus.

Infrared spectra were recorded from $4000-400 \mathrm{~cm}^{-1}$ on a Perkin Elmer Spectrum BX-59343 instrument. For detection a Smiths Detection DuraSampl IR II Diamond ATR sensor was used. The main absorption peaks are reported in $\mathrm{cm}^{-1}$.

Gas chromatographical analysis (GC) was performed with instruments of the type HewlettPackard 6890 or 5890 Series II, using a column of the type HP 5 (Hewlett-Packard, 5\% phenylmethylpolysiloxane; length: $10 \mathrm{~m}$, diameter: $0.25 \mathrm{~mm}$, film thickness $0.25 \mu \mathrm{m})$. The detection was accomplished using a flame ionization detector. Mass spectra (MS) and high resolution mass spectra (HRMS) were recorded on a Finnigan MAT 95Q or Finnigan MAT 90 instrument for electron impact ionization (EI). For the combination of gas chromatography with mass spectroscopic detection, a GC-MS of the type Hewlett-Packard 6890/MSD 5793 networking was used (column: HP 5-MS, Hewlett-Packard; 5\% phenylmethylpolysiloxane; length: $15 \mathrm{~m}$, diameter: $0.25 \mathrm{~mm}$, film thickness: $0.25 \mu \mathrm{m})$. 
Optimization of the metalation of 1,3,4-oxadiazole

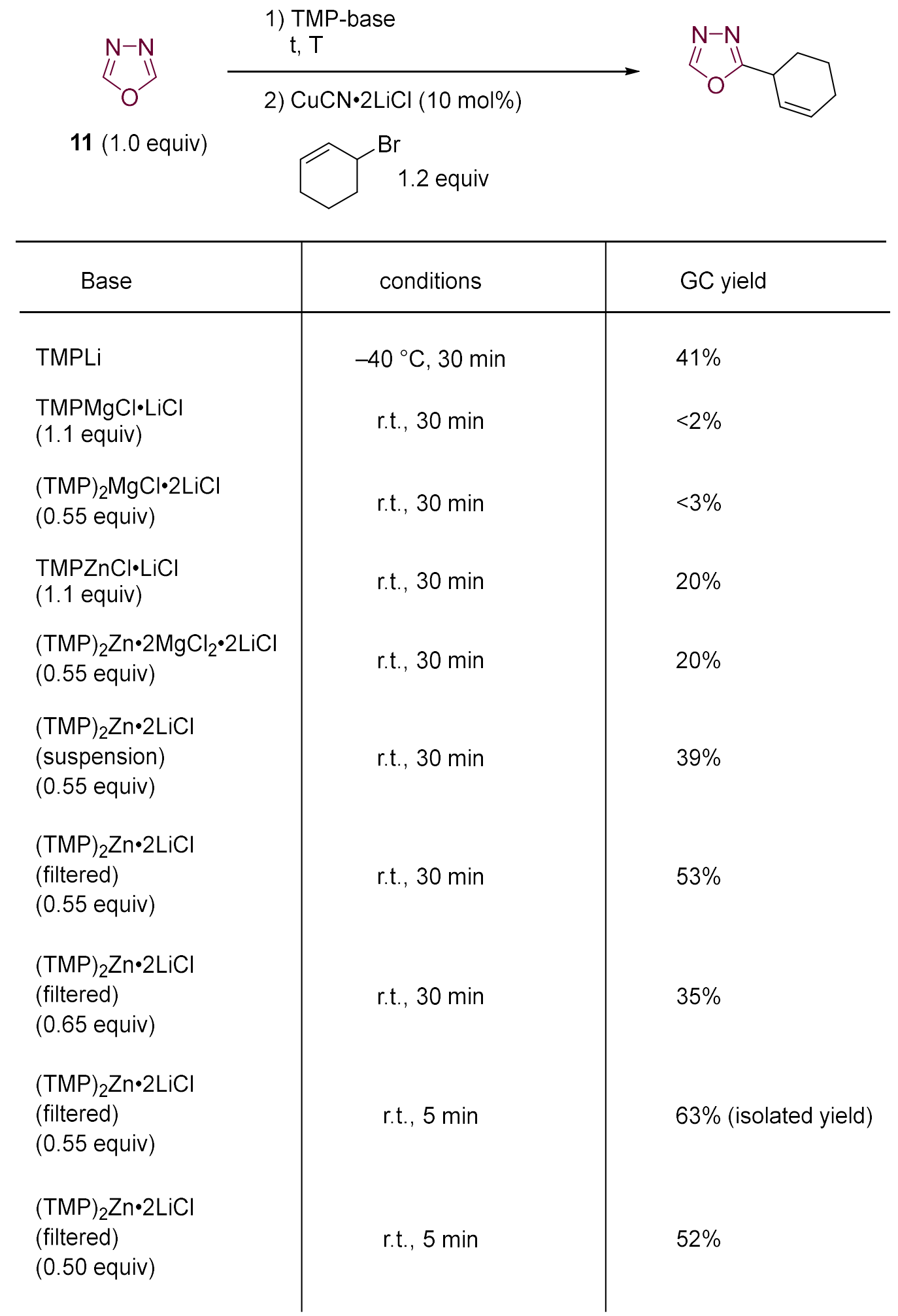




\section{Preparation of starting materials}

\section{1,3,4-Oxadiazole (11)}

$$
\begin{gathered}
0 \\
N-N \\
11
\end{gathered}
$$

1,3,4-Oxadiazole was prepared according to a literature procedure. ${ }^{3}$ Polyphosphoric acid $(108 \mathrm{~g})$ was heated to $100{ }^{\circ} \mathrm{C}$ before adding $\mathrm{P}_{2} \mathrm{O}_{5}(12 \mathrm{~g}, 42 \mathrm{mmol}, 0.31$ equiv). After stirring for $15 \mathrm{~min} N, N^{\prime}$-diformylhydrazine (12 g, $136 \mathrm{mmol}, 1.0$ equiv) was added, the resulting mixture was stirred at $100{ }^{\circ} \mathrm{C}$ for $4 \mathrm{~h}$ and then poured on ice $(100 \mathrm{~g})$. Neutralization with solid $\mathrm{NaHCO}_{3}$, extraction with $\mathrm{DCM}$, drying over $\mathrm{MgSO}_{4}$ and evaporation of the solvents in vacuo yielded 1,3,4-oxadiazole 5 (2.5 g, $36 \mathrm{mmol}, 26 \%)$ as a colorless liquid. The product was protected from light and stored at $5^{\circ} \mathrm{C}$.

${ }^{1} \mathrm{H}-\mathrm{NMR}\left(\mathrm{CDCl}_{3}, 400 \mathrm{MHz}, \mathrm{ppm}\right): \delta=8.51(\mathrm{~s}, 2 \mathrm{H})$.

${ }^{13} \mathrm{C}-\mathrm{NMR}\left(\mathrm{CDCl}_{3}, 101 \mathrm{MHz}, \mathrm{ppm}\right): \delta=152.9$.

\section{N-Substituted $1 H-1,2,4$-triazoles (12)}

$\mathrm{N}$-Substituted $1 \mathrm{H}-1,2,3$-triazoles were prepared according to a literature procedure. ${ }^{4} \mathrm{~A}$ dry and argon-flushed $250 \mathrm{~mL}$ round-bottom flask equipped with a magnetic stirrer and a septum was charged with $1 \mathrm{H}$-1,2,4-triazole $(6.91 \mathrm{~g}, 100 \mathrm{mmol})$, the respective bromide $(105 \mathrm{mmol})$ and THF (100 mL). After cooling to $4{ }^{\circ} \mathrm{C}$ 1,8-diazabicyclo(5.4.0)undec-7-ene (17.9 ml, $18.3 \mathrm{~g}, 120$ $\mathrm{mmol}$ ) was added to the solution. The mixture was stirred for $18 \mathrm{~h}$ at room temperature, quenched with water $(100 \mathrm{~mL})$ and extracted with DCM $(3 \times 80 \mathrm{~mL})$. The combined organic phases were washed with brine $(80 \mathrm{~mL})$ and dried over $\mathrm{MgSO}_{4}$. Evaporation of the solvents in vacuo and purification via column chromatography on silica gel or vacuum distillation afforded the desired $\mathrm{N}$-substituted $1 H$-1,2,3-triazole 10.

\footnotetext{
${ }^{3}$ Aitken, K. M.; Aitken, R. A. Arkivoc 2012, 5, 75.

${ }^{4}$ A. R. Katritzky, W. Kuzmierkiewiecz, J. V. Greenhill, Rec. Trav. Chim. Pays Bas 1991, 110, 369-373.
} 


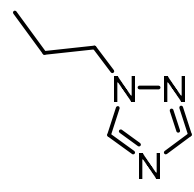

1-Propyl-1H-1,2,4-triazole was prepared using 1-bromopropane (9.6 ml, $13.0 \mathrm{~g}, 105 \mathrm{mmol})$. Purification via column chromatography ( silica gel, ihexane $/$ EtOAc $=1: 1$ ) yielded the title compound 10a (6.77 g, $60.9 \mathrm{mmol}, 61 \%)$ as a colorless liquid.

${ }^{1} \mathrm{H}-\mathrm{NMR}\left(\mathrm{CDCl}_{3}, 400 \mathrm{MHz}, \mathrm{ppm}\right): \delta=8.01(\mathrm{~s}, 1 \mathrm{H}), 7.88(\mathrm{~s}, 1 \mathrm{H}), 4.12-4.03(\mathrm{~m}, 2 \mathrm{H}), 1.93-$ $1.79(\mathrm{~m}, 2 \mathrm{H}), 0.92-0.82(\mathrm{~m}, 3 \mathrm{H})$.

${ }^{13} \mathrm{C}-\mathrm{NMR}\left(\mathrm{CDCl}_{3}, 101 \mathrm{MHz}, \mathrm{ppm}\right): \delta=151.8,142.9,51.5,23.3,11.1$.

\section{1-Benzyl-1 H-1,2,4-triazole (12b)}

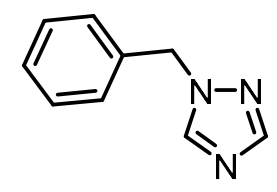

1-Benzyl-1H-1,2,4-triazole was prepared using benzyl bromide $(18.0 \mathrm{~g}, 105 \mathrm{mmol})$. Purification via column chromatography (silica gel, ihexane / EtOAc $=1: 1$ ) yielded the title compound $10 \mathrm{~b}(7.98 \mathrm{~g}, 50 \mathrm{mmol}, 50 \%)$ as a colorless solid.

${ }^{1} \mathrm{H}-\mathrm{NMR}\left(\mathrm{CDCl}_{3}, 400 \mathrm{MHz}, \mathrm{ppm}\right): \delta=8.06(\mathrm{~s}, 1 \mathrm{H}), 7.98(\mathrm{~s}, 1 \mathrm{H}), 7.46-7.30(\mathrm{~m}, 3 \mathrm{H}), 7.30-$ $7.23(\mathrm{~m}, 2 \mathrm{H}), 5.35(\mathrm{~s}, 2 \mathrm{H})$.

${ }^{13} \mathrm{C}-\mathrm{NMR}\left(\mathrm{CDCl}_{3}, 101 \mathrm{MHz}, \mathrm{ppm}\right): \delta=152.3,143.2,134.6,129.1,128.7,128.1,53.6$.

\section{1-(2-lodobenzyl)-1H-1,2,4-triazole (12c)}

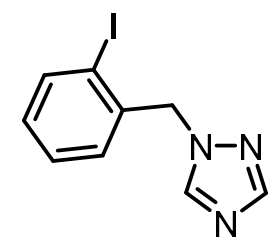

1-(2-lodobenzyl)-1H-1,2,4-triazole was prepared using 2-iodobenzyl bromide (9.57 mL, $12.9 \mathrm{~g}, 105 \mathrm{mmol})$. Purification via vacuum distillation (2 mbar, $111-114{ }^{\circ} \mathrm{C}$ ) yielded the title compound $10 \mathrm{c}(5.98 \mathrm{~g}, 53.8 \mathrm{mmol}, 54 \%)$ as a colorless oil. 
${ }^{1} \mathrm{H}-\mathrm{NMR}\left(\mathrm{CDCl}_{3}, 400 \mathrm{MHz}, \mathrm{ppm}\right): \delta=8.18(\mathrm{~s}, 1 \mathrm{H}), 8.01(\mathrm{~s}, 1 \mathrm{H}), 7.90(\mathrm{dd}, J=8.0,1.3 \mathrm{~Hz}, 1 \mathrm{H})$, 7.37 (td, $J=7.6,1.3 \mathrm{~Hz}, 1 \mathrm{H}), 7.13(\mathrm{dd}, J=7.7,1.7 \mathrm{~Hz}, 1 \mathrm{H}), 7.07(\mathrm{td}, J=7.7,1.6 \mathrm{~Hz}, 1 \mathrm{H}), 5.43$ $(\mathrm{s}, 2 \mathrm{H})$.

${ }^{13} \mathrm{C}-\mathrm{NMR}\left(\mathrm{CDCl}_{3}, 101 \mathrm{MHz}, \mathrm{ppm}\right): \delta=152.33,143.76,139.91,137.10,130.40,129.80$, 129.00, 98.74, 57.96.

\section{$(\mathrm{TMP})_{2} \mathrm{Zn} \cdot 2 \mathrm{LiCl}(8)$}

TMPH (1.7 mL, $10 \mathrm{mmol}, 1.0$ equiv) was dissolved in THF $(10 \mathrm{~mL})$ and cooled to $-40{ }^{\circ} \mathrm{C}$ before adding a solution of BuLi in hexanes $(2.3 \mathrm{M}, 4.4 \mathrm{~mL}, 10 \mathrm{~mL}, 1.0$ equiv). The resulting mixture was stirred at $0{ }^{\circ} \mathrm{C}$ for $30 \mathrm{~min}$, a solution of $\mathrm{ZnCl}_{2}$ in THF $(1.0 \mathrm{M}, 5.0 \mathrm{~mL}, 5.0 \mathrm{mmol}$, 0.50 equiv) was added, the reaction was protected from light using aluminium foil and stirred at $\mathrm{r}$.t. for 1-2 $\mathrm{h}$. The formed solids were removed using a syringe filter and the base was titrated against benzoic acid using 4-(phenylazo)-diphenylamine as an indicator.

\section{TMPMgCl·LiCl (5)}

TMPH (14.8 g, $105 \mathrm{mmol}, 1.05$ equiv) was slowly added to a solution of $i \mathrm{PrMgCl} \cdot \mathrm{LiCl}$ in THF (1.05 M, $95.2 \mathrm{~mL}, 1.00$ equiv). The resulting mixture was stirred at r.t. for 3 days before titrating the base against benzoic acid using 4-(phenylazo)-diphenylamine as an indicator.

\section{$(\mathrm{TMP})_{2} \mathrm{Zn} \cdot \mathrm{MgCl}_{2} \cdot 2 \mathrm{LiCl}(9)$}

Freshly titrated TMPMgCl$\cdot \mathrm{LiCl}$ (10 mmol, 1.0 equiv) was added dropwise to a solution of $\mathrm{ZnCl}_{2}$ in THF (1.0 M, $5.3 \mathrm{~mL}, 5.3 \mathrm{mmol}, 0.53$ equiv). The resulting mixture was protected from light using aluminium foil and stirred at r.t. for $2 \mathrm{~h}$ before titrating the base against benzoic acid using 4-(phenylazo)-diphenylamine as an indicator.

\section{TMPZnCl·LiCl (7)}

TMPH (1.7 mL, $10 \mathrm{mmol}, 1.0$ equiv) was dissolved in THF $(10 \mathrm{~mL})$ and cooled to $-40{ }^{\circ} \mathrm{C}$ before adding a solution of BuLi in hexanes $(2.3 \mathrm{M}, 4.4 \mathrm{~mL}, 10 \mathrm{~mL}, 1.0$ equiv). The resulting mixture was stirred at $0{ }^{\circ} \mathrm{C}$ for $30 \mathrm{~min}$, a solution of $\mathrm{ZnCl}_{2}$ in THF $(1.0 \mathrm{M}, 10 \mathrm{~mL}, 10 \mathrm{mmol}, 1.0$ equiv) was added, the reaction was stirred at r.t. for $1-2 \mathrm{~h}$ before titrating the base against benzoic acid using 4-(phenylazo)-diphenylamine as an indicator. 


\section{Typical procedures}

TP1: Metalation of 1,3,4-oxadiazole 11 with (TMP) ${ }_{2} \mathrm{Zn} \cdot 2 \mathrm{LiCl} 8$ followed by cross-coupling 1,3,4-Oxadiazole 11 (137 mg, $0.30 \mathrm{mmol}, 1.0$ equiv) was dissolved in THF (0.90 mL) and freshly prepared $(\mathrm{TMP})_{2} \mathrm{Zn} \cdot 2 \mathrm{LiCl} 8(0.24 \mathrm{M}, 0.68 \mathrm{~mL}, 0.17 \mathrm{mmol}, 0.55$ equiv) was added dropwise. The resulting suspension was stirred at r.t. for $5 \mathrm{~min}$ before adding $\mathrm{Pd}(\mathrm{dba})_{2}(14 \mathrm{mg}$, $0.006 \mathrm{mmol}, 3 \mathrm{~mol} \%$ ), XantPhos ( $3.5 \mathrm{mg}, 0.006 \mathrm{mmol}, 3 \mathrm{~mol} \%$ ) and the respective aryl iodide ( $0.20 \mathrm{mmol}, 0.67$ equiv). After stirring for $2 \mathrm{~h}$ a saturated aqueous solution of $\mathrm{NH}_{4} \mathrm{Cl}(1 \mathrm{~mL})$ was added. Extraction with EtOAc, drying over $\mathrm{MgSO}_{4}$, evaporation of the solvents in vacuo and purification via silica gel column chromatography yielded the desired mono-substituted 1,3,4-oxadiazole 14 .

TP2: Metalation of mono-substituted 1,3,4-oxadiazoles of type 14 with $(\mathrm{TMP})_{2} \mathrm{Zn} \cdot \mathrm{MgCl}_{2} \cdot 2 \mathrm{LiCl} 9$ followed by copper catalysed electrophilic amination

Mono-substituted 1,3,4-oxadiazole of type 14 ( $0.50 \mathrm{mmol}, 1.0$ equiv) was dissolved in THF $(1.0 \mathrm{~mL})$ and freshly prepared $(\mathrm{TMP})_{2} \mathrm{Zn} \cdot \mathrm{MgCl}_{2} \cdot 2 \mathrm{LiCl} 9(0.34 \mathrm{M}, 0.81 \mathrm{~mL}, 0.28 \mathrm{mmol}$, 0.55 equiv) was added dropwise. After stirring at r.t. for $20 \mathrm{~min}$ the mixture was added to a solution of the respective hydroxyamino benzoate $(0.35 \mathrm{mmol}, 0.70$ equiv) and copper(II) triflate $(27 \mathrm{mg}, 0.075 \mathrm{mmol}, 15 \mathrm{~mol} \%)$ in THF $(1 \mathrm{~mL})$ and stirred for additional $2 \mathrm{~h}$ at r.t. Then a saturated aqueous solution of $\mathrm{NH}_{4} \mathrm{Cl}(1 \mathrm{~mL})$ was added,. Extraction with EtOAc, drying over $\mathrm{MgSO}_{4}$, evaporation of the solvents in vacuo and purification via silica gel column chromatography yielded the desired functionalized 1,3,4-oxadiazole 15.

\section{TP3: Typical Procedure for the metalation of $N$-substituted $1 H-1,2,4$-triazoles 12 with TMPMgCI·LiCI 5}

$\mathrm{N}$-Substituted $1 \mathrm{H}-1,2,4$-triazole $12(0.50 \mathrm{mmol}, 1.0$ equiv) was dissolved in THF $(1.5 \mathrm{~mL})$ and cooled to $0{ }^{\circ} \mathrm{C}$. TMPMgCl$\cdot \mathrm{LiCl}$ solution $5(0.60 \mathrm{mmol}, 1.2$ equiv) was slowly added to the vigorously stirred solution. After $30 \mathrm{~min}$ the respective electrophile ( $0.60 \mathrm{mmol}, 1.2$ equiv) was added and the ice bath was removed. After stirring for $30 \mathrm{~min}$ the mixture was quenched with sat. aq. $\mathrm{NH}_{4} \mathrm{Cl}$ solution $(15 \mathrm{~mL})$, extracted with $\mathrm{DCM}(3 \times 15 \mathrm{~mL})$ and dried over anhydrous $\mathrm{MgSO}_{4}$. The crude product 18 was purified by flash column chromatography. 
TP4: Typical Procedure for the double functionalization of $N$-substituted $1 H-1,2,4-$ triazoles 12 with TMPMgCl$\cdot \mathrm{LiCl} 5$

$\mathrm{N}$-Substituted $1 \mathrm{H}$-1,2,4-triazole $12(0.50 \mathrm{mmol}, 1.0$ equiv) was dissolved in THF (1.5 mL) and cooled down to $0{ }^{\circ} \mathrm{C}$. TMPMgCl$\cdot \mathrm{LiCl}$ solution 5 ( $2.0 \mathrm{mmol}, 4.0$ equiv) was slowly added to the vigorously stirred solution. After $30 \mathrm{~min}$ the respective electrophile (2.2 mmol, 4.1 equiv) was added and the ice bath was removed. After 30 min the mixture was quenched with sat. aq. $\mathrm{NH}_{4} \mathrm{Cl}$ solution $(15 \mathrm{~mL})$, extracted with $\mathrm{DCM}(3 \times 15 \mathrm{~mL})$ and dried over anhydrous $\mathrm{MgSO}_{4}$. The crude product 19 was purified by flash column chromatography.

TP5: Typical Procedure for the amination of $\mathrm{N}$-substituted $1 \mathrm{H}-1,2,4$-triazoles 12 with TMPZnCl.LiCl 7

$N$-Substituted $1 \mathrm{H}-1,2,4$-triazole $12(0.50 \mathrm{mmol}, 1.0$ equiv) was dissolved in THF $(1.5 \mathrm{~mL})$ and cooled to $0{ }^{\circ} \mathrm{C}$. TMPZnCl. LiCl solution 7 ( $1 \mathrm{mmol}, 2.0$ equiv) was slowly added to the stirred solution. A dry, argon flushed Schlenk-flask equipped with a magnetic stirring bar and a septum was charged with amine benzoate $(0.6 \mathrm{mmol}, 1.2$ equiv), copper (II) triflate (36 mg, $0.1 \mathrm{mmol}, 20 \mathrm{~mol} \%)$ and THF $(2.0 \mathrm{~mL})$. After $30 \mathrm{~min}$ the metalated species was slowly added to the vigorously stirred solution. After $2 \mathrm{~h}$ the mixture was quenched with sat. aq. $\mathrm{NH}_{4} \mathrm{Cl}$ solution $(15 \mathrm{~mL})$, extracted with $\mathrm{DCM}(3 \times 15 \mathrm{~mL})$ and dried over anhydrous $\mathrm{MgSO}_{4}$. The crude product 18 was purified by flash column chromatography.

\section{TP6: Typical Procedure for the Cross-Coupling of $\mathrm{N}$-substituted $1 \mathrm{H}-1,2,4$-triazoles 12}

$\mathrm{N}$-Substituted $1 \mathrm{H}$-1,2,4-triazole $12(0.50 \mathrm{mmol}, 1.0$ equiv) was dissolved in THF (1.5 mL) and cooled to $0{ }^{\circ} \mathrm{C}$. TMPZnCl- $\mathrm{LiCl}$ solution $7(0.65 \mathrm{mmol}, 1.3$ equiv $)$ was slowly added to the stirred solution. A dry, argon flushed Schlenk-flask equipped with a magnetic stirring bar and a septum was charged with the respective aromatic bromide or iodide ( $0.6 \mathrm{mmol}, 1.2$ equiv), $\mathrm{Pd}(\mathrm{OAc})_{2}(5.6 \mathrm{mg}, 0.025 \mathrm{mmol}, 5 \mathrm{~mol} \%)$, SPhos $(20.5 \mathrm{mg}, 0.050 \mathrm{mmol}, 10 \mathrm{~mol} \%$ ) and THF $(1.0 \mathrm{~mL})$. After $30 \mathrm{~min}$ the metalated species was slowly added to the vigorously stirred solution. The reaction mixture was stirred at $40{ }^{\circ} \mathrm{C}$ for $18 \mathrm{~h}$, quenched with sat. aq. $\mathrm{NH}_{4} \mathrm{Cl}$ solution $(15 \mathrm{~mL})$, extracted with $\mathrm{DCM}(3 \times 15 \mathrm{~mL})$ and dried over anhydrous $\mathrm{MgSO}_{4}$. The crude product 18 was purified by flash column chromatography. 


\section{Products:}

2-Phenyl-1,3,4-oxadiazole (14a)

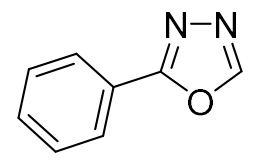

2-Phenyl-1,3,4-oxadiazole was prepared according to TP1 using phenyl iodide (41 mg, $0.20 \mathrm{mmol}$ ). Purification via column chromatography (silica gel, ihexane $/$ EtOAc $=4: 1$ ) yielded the title compound $14 \mathrm{a}(25 \mathrm{mg}, 0.17 \mathrm{mmol}, 86 \%)$ as colorless crystals.

${ }^{1} \mathrm{H}-\mathrm{NMR}\left(\mathrm{CDCl}_{3}, 400 \mathrm{MHz}, \mathrm{ppm}\right): \delta=8.48(\mathrm{~s}, 1 \mathrm{H}), 8.12-8.02(\mathrm{~m}, 2 \mathrm{H}), 7.60-7.46(\mathrm{~m}, 3 \mathrm{H})$.

${ }^{13} \mathrm{C}-\mathrm{NMR}\left(\mathrm{CDCl}_{3}, 101 \mathrm{MHz}, \mathrm{ppm}\right): \delta=164.8,152.7,132.0,129.1,127.1,123.5$.

MS (70 eV, El) m/z (\%): 146 (100) [M] ${ }^{+}, 105$ (42), 91 (11), 90 (32), 89 (19), 77 (16).

IR (ATR) $\widetilde{v}\left(\mathbf{c m}^{-1}\right): 3231,3131,1706,1663,1609,1589,1555,1514,1481,1449,1340,1288$, 1234, 1179, 1105, 1077, 1063, 1025, 1002, 955, 944, 927, 849, 777, 709, 687.

HRMS (EI) calculated for $\mathrm{C}_{8} \mathrm{H}_{6} \mathrm{~N}_{2} \mathrm{O}^{+\cdot}$ : 146.0475 , found $146.0475[\mathrm{M}]^{+}$.

mp: $33.8-35.1^{\circ} \mathrm{C}$.

Ethyl 4-(1,3,4-oxadiazol-2-yl)benzoate (14b)

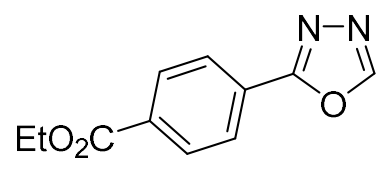

Ethyl 4-(1,3,4-oxadiazol-2-yl)benzoate was prepared according to TP1 using ethyl 4-iodobenzoate $(55 \mathrm{mg}, 0.20 \mathrm{mmol}$ ). Purification via column chromatography (silica gel, ihexane $/$ EtOAc $=3: 1)$ yielded the title compound $14 \mathrm{~b}(43 \mathrm{mg}, 0.197 \mathrm{mmol}, 98 \%)$ as a colorless solid.

${ }^{1} \mathrm{H}-\mathrm{NMR}\left(\mathrm{CDCl}_{3}, 400 \mathrm{MHz}, \mathrm{ppm}\right): \delta=8.53(\mathrm{~s}, 1 \mathrm{H}), 8.21-8.13(\mathrm{~m}, 4 \mathrm{H}), 4.41(\mathrm{q}, \mathrm{J}=7.1 \mathrm{~Hz}$, $2 \mathrm{H}), 1.42(\mathrm{t}, \mathrm{J}=7.1 \mathrm{~Hz}, 3 \mathrm{H})$.

${ }^{13} \mathrm{C}-\mathrm{NMR}\left(\mathrm{CDCl}_{3}, 101 \mathrm{MHz}, \mathrm{ppm}\right): \delta=165.6,164.1,153.0,133.5,130.3,127.1,127.0,61.6$, 14.3.

MS (70 eV, El) m/z (\%): 218 (7) [M] ${ }^{+}, 190$ (64), 174 (10), 173 100), 149 (14), 145 (14), 118 (8), 90 (8). 
IR (ATR) $\widetilde{v}\left(\mathbf{c m}^{-1}\right): 3124,1701,1553,1477,1414,1370,1310,1277,1178,1131,1105,1069$, $1013,955,874,847,779,714$.

HRMS (EI) calculated for $\mathrm{C}_{11} \mathrm{H}_{11} \mathrm{~N}_{2} \mathrm{O}_{3}{ }^{+}:$218.0686, found $218.0688[\mathrm{M}]^{+}$.

mp: $93.8-95.5^{\circ} \mathrm{C}$.

\section{2-(4-Chlorophenyl)-1,3,4-oxadiazole (14c)}

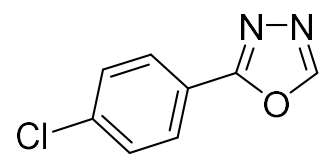

2-(4-Chlorophenyl)-1,3,4-oxadiazole was prepared according to TP1 using 1-chloro-4iodobenzene (48 mg, $0.20 \mathrm{mmol}$ ). Purification via column chromatography (silica gel, ihexane / EtOAc $=4: 1)$ yielded the title compound $14 \mathrm{c}(31 \mathrm{mg}, 0.17 \mathrm{mmol}, 86 \%)$ as a slightly brown solid.

${ }^{1} \mathrm{H}-\mathrm{NMR}\left(\mathrm{CDCl}_{3}, 400 \mathrm{MHz}, \mathrm{ppm}\right): \delta=8.48(\mathrm{~s}, 1 \mathrm{H}), 8.02(\mathrm{~d}, \mathrm{~J}=8.6 \mathrm{~Hz}, 2 \mathrm{H}), 7.50(\mathrm{~d}$, $\mathrm{J}=8.6 \mathrm{~Hz}, 2 \mathrm{H})$.

${ }^{13} \mathrm{C}-\mathrm{NMR}\left(\mathrm{CDCl}_{3}, 101 \mathrm{MHz}, \mathrm{ppm}\right): \delta=164.0,152.7,138.4,129.6,128.4,121.9$.

MS (70 eV, EI) m/z (\%): 182 (35), 181 (13), 180 (100) [M] ${ }^{+}, 141$ (24), 139 (95), 137 (20), 125 (17), 124 (20), 111 (22), 89 (29), 85 (14), 75 (21), 71 (19), 69 (10), 57 (21), 43 (17).

IR (ATR) $\widetilde{v}\left(\mathbf{c m}^{-1}\right): 3148,2921,1983,1716,1605,1583,1549,1513,1480,1406,1332,1278$ 1215, 1172, 1114, 1091, 1062, 1009, 971, 959, 951, 941, 859, 830, 741, 730, 693.

HRMS (EI) calculated for $\mathrm{C}_{8} \mathrm{H}_{5} \mathrm{CIN}_{2} \mathrm{O}^{+*}: 180.0085$, found 180.0081 [M] ${ }^{+}$.

$\mathrm{mp}: 132.7-134.6^{\circ} \mathrm{C}$.

4-(1,3,4-Oxadiazol-2-yl)benzonitrile (14d)<smiles>N#Cc1ccc(-c2nnco2)cc1</smiles>

4-(1,3,4-Oxadiazol-2-yl)benzonitrile was prepared according to TP1 using 4-iodobenzonitrile $(46 \mathrm{mg}, 0.20 \mathrm{mmol})$. Purification via column chromatography (silica gel, ihexane $/$ EtOAc $=$ $6: 4$ ) yielded the title compound $14 \mathrm{~d}(25 \mathrm{mg}, 0.15 \mathrm{mmol}, 73 \%$ ) as a colorless solid. 
${ }^{1} \mathrm{H}-\mathrm{NMR}\left(\mathrm{CDCl}_{3}, 400 \mathrm{MHz}, \mathrm{ppm}\right): \delta=8.50(\mathrm{~s}, 1 \mathrm{H}), 8.15(\mathrm{~d}, \mathrm{~J}=8.7 \mathrm{~Hz}, 2 \mathrm{H}), 7.77(\mathrm{~d}$, $\mathrm{J}=8.7 \mathrm{~Hz}, 2 \mathrm{H})$.

${ }^{13} \mathrm{C}-\mathrm{NMR}\left(\mathrm{CDCl}_{3}, 101 \mathrm{MHz}, \mathrm{ppm}\right): \delta=163.3,153.3,133.0,127.6,127.3,117.8,115.6$.

MS (70 eV, El) m/z (\%): $171(69)\left[\mathrm{M}^{+}, 130(57), 128\right.$ (16), $116(37), 115$ (100), $114(36)$, 91 (11), 88 (13), 76 (17), 75 (20), 62 (10).

IR (ATR) $\tilde{v}\left(\mathrm{~cm}^{-1}\right):$ 3154, 2922, 2228, 1581, 1551, 1501, 1489, 1405, 1282, 1225, 1091, 1068 , 1015, 956, 867, 839, 736, 698.

HRMS (EI) calculated for $\mathrm{C}_{9} \mathrm{H}_{5} \mathrm{~N}_{3} \mathrm{O}^{++}: 171.0427$, found $171.0425[\mathrm{M}]^{+}$.

mp: $145.6-147.7^{\circ} \mathrm{C}$.

\section{2-(4-(Trifluoromethyl)phenyl)-1,3,4-oxadiazole (14e)}

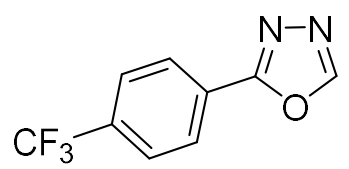

2-(4-(trifluoromethyl)phenyl)-1,3,4-oxadiazole was prepared according to TP1 using 1-iodo-4(trifluoromethyl)benzene (55 mg, $0.20 \mathrm{mmol}$ ). Purification via column chromatography (silica gel, ihexane $/$ EtOAc $=4: 1)$ yielded the title compound $14 \mathrm{e}(35 \mathrm{mg}, 0.16 \mathrm{mmol}, 82 \%)$ as a colorless solid.

${ }^{1} \mathrm{H}-\mathrm{NMR}\left(\mathrm{CDCl}_{3}, 400 \mathrm{MHz}, \mathrm{ppm}\right): \delta=8.54(\mathrm{~s}, 1 \mathrm{H}), 8.22(\mathrm{~d}, \mathrm{~J}=7.7 \mathrm{~Hz}, 2 \mathrm{H}), 7.79(\mathrm{~d}$, $\mathrm{J}=8.4 \mathrm{~Hz}, 2 \mathrm{H})$.

${ }^{13} \mathrm{C}-N M R\left(\mathrm{CDCl}_{3}, 101 \mathrm{MHz}, \mathrm{ppm}\right): \delta=163.7,153.1,133.7$ (q, $\left.\mathrm{J}=33.0 \mathrm{~Hz}\right), 127.5,126.7$, $126,2(q, J=3.8 \mathrm{~Hz}), 123.5$ (q, J = 273.1 Hz).

MS (70 eV, El) m/z (\%): 214 (100) [M] ${ }^{+}, 195$ (13), 173 (69), 167 (10), 158 (71), 145 (38), 138 (9).

IR (ATR) $\widetilde{v}\left(\mathbf{c m}^{-1}\right):$ 3153, 2923, 1562, 1516, 1414, 1319, 1224, 1186, 1157, 1111, 1071, 1056, 1010, 947, 867, 847, 748, 705.

HRMS (EI) calculated for $\mathrm{C}_{9} \mathrm{H}_{5} \mathrm{~F}_{3} \mathrm{~N}_{2} \mathrm{O}^{++}:$214.0348, found 214.0348 [M] ${ }^{+}$.

mp: $114.1-115.9^{\circ} \mathrm{C}$. 


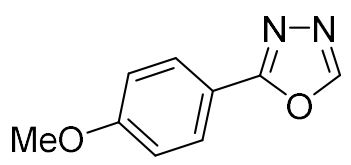

2-(4-Methoxyphenyl)-1,3,4-oxadiazole was prepared according to TP1 using 1-iodo-4methoxybenzene $(47 \mathrm{mg}, 0.20 \mathrm{mmol})$. The cross-coupling was conducted at $50{ }^{\circ} \mathrm{C}$ using $\mathrm{Pd}\left(\mathrm{PPh}_{3}\right)_{4}(17 \mathrm{mg}, 0.015 \mathrm{mmol}, 7.5 \mathrm{~mol} \%)$ as a catalyst. Purification via column chromatography (silica gel, ihexane $/$ EtOAc $=6: 4$ ) yielded the title compound $14 \mathrm{f}(32 \mathrm{mg}$, $0.18 \mathrm{mmol}, 90 \%$ ) as a colorless solid.

${ }^{1} \mathrm{H}-\mathrm{NMR}\left(\mathrm{CDCl}_{3}, 400 \mathrm{MHz}, \mathrm{ppm}\right): \delta=8.41(\mathrm{~s}, 1 \mathrm{H}), 8.01(\mathrm{~d}, \mathrm{~J}=8.9 \mathrm{~Hz}, 2 \mathrm{H}), 7.01$ (d, $\mathrm{J}=8.9 \mathrm{~Hz}, 2 \mathrm{H}), 3.88(\mathrm{~s}, 3 \mathrm{H})$.

${ }^{13} \mathrm{C}-\mathrm{NMR}\left(\mathrm{CDCl}_{3}, 101 \mathrm{MHz}, \mathrm{ppm}\right): \delta=164.7,162.5,152.2,128.9,116.0,114.5,55.5$.

MS (70 eV, El) m/z (\%): $176(100)\left[\mathrm{M}^{+}, 136\right.$ (8), 135 (95), 91 (6), 77 (7).

IR (ATR) $\tilde{v}\left(\mathbf{c m}^{-1}\right): 3126,2922,2851,1718,1612,1587,1559,1517,1494,1456,1438,1422$, 1302, 1257, 1179, 1122, 1110, 1097, 1066, 1017, 973, 959, 939, 860, 832, 818, 797, 738, 702.

HRMS (EI) calculated for $\mathrm{C}_{9} \mathrm{H}_{8} \mathrm{~N}_{2} \mathrm{O}_{2}{ }^{++}: 176.0580$, found $176.0580[\mathrm{M}]^{+}$.

mp: $64.6-66.2^{\circ} \mathrm{C}$.

N,N-Dimethyl-4-(1,3,4-oxadiazol-2-yl)aniline (14g)

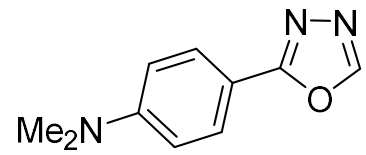

N,N-Dimethyl-4-(1,3,4-oxadiazol-2-yl)aniline was prepared according to TP1 using 4-iodo$\mathrm{N}, \mathrm{N}$-dimethylaniline $(49 \mathrm{mg}, 0.20 \mathrm{mmol})$. The cross-coupling was conducted at $50{ }^{\circ} \mathrm{C}$ using $\mathrm{Pd}\left(\mathrm{PPh}_{3}\right)_{4}(17 \mathrm{mg}, \quad 0.015 \mathrm{mmol}, 7.5 \mathrm{~mol} \%)$ as a catalyst. Purification via column chromatography (silica gel, ihexane / EtOAc $=7: 3$ ) yielded the title compound $\mathbf{1 4 g}(24 \mathrm{mg}$, $0.13 \mathrm{mmol}, 63 \%$ ) as a colorless solid.

${ }^{1} \mathrm{H}-\mathrm{NMR}\left(\mathrm{CDCl}_{3}, 400 \mathrm{MHz}, \mathrm{ppm}\right): \delta=8.35(\mathrm{~s}, 1 \mathrm{H}), 7.91(\mathrm{~d}, \mathrm{~J}=9.0 \mathrm{~Hz}, 2 \mathrm{H}), 6.74$ (d, $\mathrm{J}=9.0 \mathrm{~Hz}, 2 \mathrm{H}), 3.05(\mathrm{~s}, 6 \mathrm{H})$.

${ }^{13} \mathrm{C}-\mathrm{NMR}\left(\mathrm{CDCl}_{3}, 101 \mathrm{MHz}, \mathrm{ppm}\right): \delta=165.5,152.5,151.7,128.6,111.7,110.6,40.2$ 
MS (70 eV, El) m/z (\%): 189 (100) [M] ${ }^{+}, 188(60), 161(7), 148(30), 145(13), 132(13), 118$ (7).

$\operatorname{IR}(\mathrm{ATR}) \tilde{v}\left(\mathrm{~cm}^{-1}\right)$ :

HRMS (El) calculated for $\mathrm{C}_{10} \mathrm{H}_{11} \mathrm{~N}_{3} \mathrm{O}^{+*}: 189.0897$, found 189.0896 [M] ${ }^{+}$.

mp: $134.2-136.1^{\circ} \mathrm{C}$.

2-(3-Nitrophenyl)-1,3,4-oxadiazole (14h)

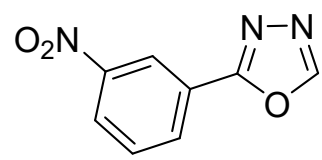

2-(3-Nitrophenyl)-1,3,4-oxadiazole was prepared according to TP1 using 1-iodo-3nitrobenzene (50 mg, $0.20 \mathrm{mmol}$ ). Purification via column chromatography (silica gel, ihexane / EtOAc $=6: 4)$ yielded the title compound $14 \mathrm{~h}(37 \mathrm{mg}, 0.19 \mathrm{mmol}, 97 \%)$ as a colorless solid.

${ }^{1} \mathrm{H}-\mathrm{NMR}\left(\mathrm{CDCl}_{3}, 400 \mathrm{MHz}, \mathrm{ppm}\right): \delta=8.91$ (t, J = 2.0 Hz, $1 \mathrm{H}$ ), $8.58(\mathrm{~s}, 1 \mathrm{H}), 8.45$ (ddd, J = 7.8, 1.6, $1.0 \mathrm{~Hz}, 1 \mathrm{H}), 8.42(\mathrm{ddd}, \mathrm{J}=8.3,2.3,1.1 \mathrm{~Hz}, 1 \mathrm{H}), 7.76(\mathrm{t}, \mathrm{J}=8.0 \mathrm{~Hz}, 1 \mathrm{H})$.

${ }^{13} \mathrm{C}-\mathrm{NMR}\left(\mathrm{CDCl}_{3}, 101 \mathrm{MHz}, \mathrm{ppm}\right): \delta=163.0,153.3,148.6,132.7,130.6,126.5,125.1,122.1$. MS (70 eV, EI) $\mathbf{m / z}(\%): 191$ (86) [M] ${ }^{+}, 150$ (49), 145 (15), $118(21), 117(14), 104$ (13), 90 (77), 89 (100), 88 (14), 87 (18), 86 (16), 76 (42), 75 (39), 74 (26), 63 (47), 62 (19), 50 (12), 46 (12).

IR (ATR) $\widetilde{v}\left(\mathbf{c m}^{-1}\right): 3160,3092,2923,2853,1620,1591,1556,1530,1508,1475,1352,1253$, 1238, 1166, 1122, 1104, 1061, 1001, 976, 963, 953, 906, 883, 871, 856, 812, 753, 739, 711, 668.

HRMS (EI) calculated for $\mathrm{C}_{8} \mathrm{H}_{5} \mathrm{~N}_{3} \mathrm{O}_{3}{ }^{+}:$191.0325, found $191.0324[\mathrm{M}]^{+}$.

mp: $127.6-129.3^{\circ} \mathrm{C}$.

\section{2-(2-Fluorophenyl)-1,3,4-oxadiazole (14i)}

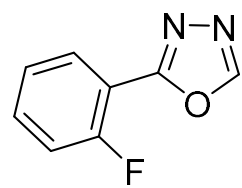

2-(2-Fluorophenyl)-1,3,4-oxadiazole was prepared according to TP1 using 1-fluoro-2iodobenzene (44 mg, $0.20 \mathrm{mmol}$ ). Purification via column chromatography (silica gel, ihexane 
/ EtOAc $=4: 1)$ yielded the title compound $14 \mathrm{i}(29 \mathrm{mg}, 0.18 \mathrm{mmol}, 88 \%)$ as a slightly brown liquid.

${ }^{1} \mathrm{H}-\mathrm{NMR}\left(\mathrm{CDCl}_{3}, 400 \mathrm{MHz}, \mathrm{ppm}\right): \delta=8.54(\mathrm{~s}, 1 \mathrm{H}), 8.08(\mathrm{ddd}, \mathrm{J}=7.9,7.1,1.8 \mathrm{~Hz}, 1 \mathrm{H}), 7.59$ - $7.51(\mathrm{~m}, 1 \mathrm{H}), 7.31$ (td, J = 7.6, $1.1 \mathrm{~Hz}, 1 \mathrm{H}), 7.26$ (ddd, J = 10.5, 8.3, $1.1 \mathrm{~Hz}, 1 \mathrm{H}$ ).

${ }^{13} \mathrm{C}-N M R\left(\mathrm{CDCl}_{3}, 101 \mathrm{MHz}, \mathrm{ppm}\right): \delta=161.7$ (d, J = 4.9 Hz), 160.1 (d, J = 258.6 Hz), 153.0 (d, J = 1.2 Hz), 133.9 (d, J = 8.5 Hz), 129.9, 124.8 (d, J = 3.8 Hz), 117.1 (d, J = 20.8 Hz), 112.0 $(\mathrm{d}, \mathrm{J}=11.7 \mathrm{~Hz})$.

MS (70 eV, EI) m/z (\%): 164 (100) [M] ${ }^{+}, 123$ (36), 109 (20), 108 (57), 107 (24).

IR (ATR) $\tilde{v}\left(\mathbf{c m}^{-1}\right): 3117,1621,1591,1550,1515,1492,1472,1442,1405,1346,1272,1262$, $1223,1159,1102,1069,1053,1027,955,856,820,765,740,700,663$.

HRMS (EI) calculated for $\mathrm{C}_{8} \mathrm{H}_{5} \mathrm{FN}_{2} \mathrm{O}^{+*}: 164.0380$, found 164.0379 [M] $^{+}$.

\section{2-(Quinolin-6-yl)-1,3,4-oxadiazole (14j)}

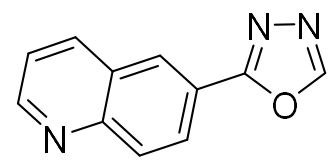

2-(Quinolin-6-yl)-1,3,4-oxadiazole was prepared according to TP1 using 6-iodoquinoline (51 mg, $0.20 \mathrm{mmol}$ ). Purification via column chromatography (silica gel, EtOAc) yielded the title compound $14 \mathrm{j}$ (36 mg, $0.19 \mathrm{mmol}, 92 \%$ ) as a slightly yellow solid.

${ }^{1} \mathrm{H}-\mathrm{NMR}\left(\mathrm{CDCl}_{3}, 400 \mathrm{MHz}, \mathrm{ppm}\right): \delta=9.01(\mathrm{dd}, \mathrm{J}=4.3,1.7 \mathrm{~Hz}, 1 \mathrm{H}), 8.57(\mathrm{~d}, \mathrm{~J}=1.9 \mathrm{~Hz}, 1 \mathrm{H})$, $8.55(\mathrm{~s}, 1 \mathrm{H}), 8.37$ (dd, J = 8.8, $1.9 \mathrm{~Hz}, 1 \mathrm{H}), 8.28(\mathrm{~d}, \mathrm{~J}=8.4 \mathrm{~Hz}, 1 \mathrm{H}), 8.23(\mathrm{~d}, \mathrm{~J}=8.9 \mathrm{~Hz}, 1$ H), 7.50 (dd, J = 8.3, $4.2 \mathrm{~Hz}, 1 \mathrm{H}$ ).

${ }^{13} \mathrm{C}-\mathrm{NMR}\left(\mathrm{CDCl}_{3}, 101 \mathrm{MHz}, \mathrm{ppm}\right): \delta=164.5,153.1,152.5,149.4,137.0,130.9,128.0,127.7$, 127.1, 122.4, 121.6 .

MS (70 eV, El) m/z (\%): 197 (98) [M] ${ }^{+}, 157$ (10), 156 (100), 141 (10), 140 (12), 128 (27).

IR (ATR) $\widetilde{v}\left(\mathbf{c m}^{-1}\right):$ 3351, 3080, 2923, 2852, 1628, 1578, 1549, 1509, 1495, 1438, 1367, 1331 , 1310, 1249, 1187, 1127, 1089, 1059, 1037, 977, 958, 939, 911, 889, 850, 834, 798, 768, 723.

HRMS (El) calculated for $\mathrm{C}_{11} \mathrm{H}_{7} \mathrm{~N}_{3} \mathrm{O}^{+}:$: 197.0584, found $197.0582[\mathrm{M}]^{+}$.

mp: $119.1-121.5^{\circ} \mathrm{C}$. 


\section{2-(Thiophen-2-yl)-1,3,4-oxadiazole (14k)}

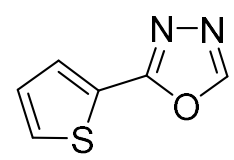

2-(Thiophen-2-yl)-1,3,4-oxadiazole was prepared according to TP1 using 2-iodothiophene $(42 \mathrm{mg}, 0.20 \mathrm{mmol})$. Purification via column chromatography (silica gel, ihexane $/$ EtOAc = $4: 1)$ yielded the title compound $14 \mathbf{k}(28 \mathrm{mg}, 0.18 \mathrm{mmol}, 92 \%)$ as a slightly brown liquid.

${ }^{1} \mathrm{H}-\mathrm{NMR}\left(\mathrm{CDCl}_{3}, 400 \mathrm{MHz}, \mathrm{ppm}\right): \delta=8.40(\mathrm{~s}, 1 \mathrm{H}), 7.79$ (dd, J = 3.8, $\left.1.2 \mathrm{~Hz}, 1 \mathrm{H}\right), 7.57$ (dd, $\mathrm{J}=5.0,1.2 \mathrm{~Hz}, 1 \mathrm{H}), 7.18(\mathrm{dd}, \mathrm{J}=5.0,3.7 \mathrm{~Hz}, 1 \mathrm{H})$.

${ }^{13} \mathrm{C}-\mathrm{NMR}\left(\mathrm{CDCl}_{3}, 101 \mathrm{MHz}, \mathrm{ppm}\right): \delta=161.0,152.0,130.6,130.3,128.3,124.7$.

MS (70 eV, El) m/z (\%): $152(100)\left[\mathrm{M}^{+}, 110(49), 96(22), 70(6)\right.$

IR (ATR) $\widetilde{v}\left(\mathbf{c m}^{-1}\right):$ 3228, 3106, 3000, 1703, 1652, 1593, 1578, 1520, 1490, 1419, 1372, 1355, 1297, 1233, 1214, 1096, 1061, 1010, 953; 919, 851, 720.

HRMS (El) calculated for $\mathrm{C}_{6} \mathrm{H}_{4} \mathrm{~N}_{2} \mathrm{OS}^{+}:$152.0039, found 152.0038 [M] ${ }^{+}$.

\section{4-(5-Phenyl-1,3,4-oxadiazol-2-yl)morpholine (16a)}

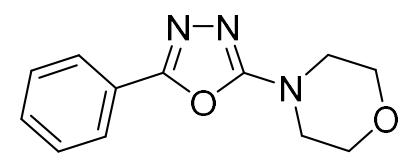

4-(5-Phenyl-1,3,4-oxadiazol-2-yl)morpholine was prepared according to TP2 using 2-phenyl1,3,4-oxadiazole 14a (73 $\mathrm{mg}, 0.50 \mathrm{mmol}$ ) and morpholino benzoate $(73 \mathrm{mg}, 0.35 \mathrm{mmol})$. Purification via column chromatography (silica gel, ihexane $/$ EtOAc $=3: 1$ ) yielded the title compound 9 a (79 mg, $0.34 \mathrm{mmol}, 98 \%$ ) as a colorless oil.

${ }^{1} \mathrm{H}-\mathrm{NMR}\left(\mathrm{CDCl}_{3}, 400 \mathrm{MHz}, \mathrm{ppm}\right): \delta=7.88-7.78(\mathrm{~m}, 2 \mathrm{H}), 7.38(\mathrm{dq}, J=6.6,2.8,1.8 \mathrm{~Hz}, 3 \mathrm{H})$, $3.82-3.73(\mathrm{~m}, 4 \mathrm{H}), 3.53(\mathrm{dd}, J=5.8,3.9 \mathrm{~Hz}, 4 \mathrm{H})$.

${ }^{13} \mathrm{C}$-NMR ( $\left.\mathrm{CDCl}_{3}, 101 \mathrm{MHz}, \mathrm{ppm}\right): \delta=164.0,159.6,130.7,128.9$ (2C), $125.9(2 \mathrm{C}), 124.4$, $66.0(2 \mathrm{C}), 46.2(2 \mathrm{C})$.

MS (70 eV, EI) m/z (\%): $231(80)\left[\mathrm{M}^{+}, 207\right.$ (25), 135 (100), 70 (6).

IR (ATR) $\widetilde{v}\left(\mathrm{~cm}^{-1}\right):$ 2398, 2377, 1798, 1721, 1355, 1348, 1250, 688.

HRMS (EI) calculated for $\mathrm{C}_{12} \mathrm{H}_{13} \mathrm{~N}_{3} \mathrm{O}_{2}{ }^{+}:$231.1008, found 231.1003 [M] ${ }^{+}$. 
N,N-Dimethyl-4-(5-morpholino-1,3,4-oxadiazol-2-yl)aniline (16b)<smiles>CNc1ccc(-c2nnc(N3CCOCC3)o2)cc1</smiles>

N,N-Dimethyl-4-(5-morpholino-1,3,4-oxadiazol-2-yl)aniline was prepared according to TP2 using $N, N$-dimethyl-4-(1,3,4-oxadiazol-2-yl)aniline $14 \mathrm{~g}(95 \mathrm{mg}, 0.50 \mathrm{mmol})$ and morpholino benzoate (73 mg, $0.35 \mathrm{mmol}$ ). Purification via column chromatography (silica gel, ihexane / EtOAc $=3: 1)$ yielded the title compound $16 \mathrm{~b}(71 \mathrm{mg}, 0.26 \mathrm{mmol}, 74 \%)$ as a colorless oil.

${ }^{1} \mathrm{H}-\mathrm{NMR}\left(\mathrm{CDCl}_{3}, 400 \mathrm{MHz}, \mathrm{ppm}\right): \delta=7.83-7.63(\mathrm{~m}, 2 \mathrm{H}), 6.78-6.62(\mathrm{~m}, 2 \mathrm{H}), 3.88-3.72$ (m, 4H), $3.66-3.48(\mathrm{~m}, 4 \mathrm{H}), 3.01(\mathrm{~s}, 6 \mathrm{H})$.

${ }^{13} \mathrm{C}-\mathrm{NMR}\left(\mathrm{CDCl}_{3}, 101 \mathrm{MHz}, \mathrm{ppm}\right): \delta=163.6,160.5,151.8,127.3(2 \mathrm{C}), 111.7(2 \mathrm{C}), 66.1(2 \mathrm{C})$, $46.4(2 \mathrm{C}), 40.2(2 \mathrm{C})$.

MS (70 eV, El) $\mathbf{m} / \mathbf{z}(\%): 274(86)\left[\mathrm{M}^{+}, 207\right.$ (35), 135 (100), $120(12)$

IR (ATR) $\widetilde{v}\left(\mathbf{c m}^{-1}\right):$ 2972, 2917, 2893, 2857, 1605, 1572, 1510, 1452, 1445, 1432, 1364, 1272 , 1258, 1228, 1192, 1170, 1117, 1071, 1062, 951, 945, 909, 820, 761, 725, 704.

HRMS (EI) calculated for $\mathrm{C}_{14} \mathrm{H}_{18} \mathrm{~N}_{4} \mathrm{O}_{2}{ }^{++}: 274.1430$, found $274.1438[\mathrm{M}]^{+}$.

4-(5-(4-Chlorophenyl)-1,3,4-oxadiazol-2-yl)morpholine (16c)<smiles>Clc1ccc(-c2nnc(N3CCOCC3)o2)cc1</smiles>

4-(5-(4-Chlorophenyl)-1,3,4-oxadiazol-2-yl)morpholine was prepared according to TP2 using 2-(4-chlorophenyl)-1,3,4-oxadiazole 14c $(90 \mathrm{mg}, 0.50 \mathrm{mmol})$ and morpholino benzoate $(73 \mathrm{mg}, 0.35 \mathrm{mmol})$. Purification via column chromatography (silica gel, ihexane $/ \mathrm{EtOAc}=$ $3: 1)$ yielded the title compound $16 \mathrm{c}(74 \mathrm{mg}, 0.28 \mathrm{mmol}, 80 \%)$ as a colorless oil.

${ }^{1} \mathrm{H}-\mathrm{NMR}\left(\mathrm{CDCl}_{3}, 400 \mathrm{MHz}, \mathrm{ppm}\right): \delta=7.86-7.71(\mathrm{~m}, 2 \mathrm{H}), 7.45-7.31(\mathrm{~m}, 2 \mathrm{H}), 3.84-3.70$ $(\mathrm{m}, 4 \mathrm{H}), 3.65-3.48(\mathrm{~m}, 4 \mathrm{H})$.

${ }^{13} \mathrm{C}-N M R\left(\mathrm{CDCl}_{3}, 101 \mathrm{MHz}, \mathrm{ppm}\right): \delta=164.2,158.9,136.8,129.4(2 \mathrm{C}), 127.2(2 \mathrm{C}), 123.0$, $66.1(2 \mathrm{C}), 46.3(2 \mathrm{C})$.

MS (70 eV, EI) $m / z$ (\%): 265 (78) [M] ${ }^{+}, 207$ (29), 135 (100), 70 (9). 
IR (ATR) $\widetilde{v}\left(\mathbf{c m}^{-1}\right):$ 2922, 1706, 1637, 1618, 1614, 1603, 1577, 1485, 1440, 1434, 1421, 1396, $1390,1359,1273,1258,1221,1120,1114,1092,1060,1012,912,837,732$.

HRMS (El) calculated for $\mathrm{C}_{12} \mathrm{H}_{12} \mathrm{CIN}_{3} \mathrm{O}_{2}^{+}:$265.0618, found 265.0615 [M] ${ }^{+}$.

4-(5-(4-(Trifluoromethyl)phenyl)-1,3,4-oxadiazol-2-yl)morpholine (16d)<smiles>FC(F)(F)c1ccc(-c2nnc(N3CCOCC3)o2)cc1</smiles>

4-(5-(4-(Trifluoromethyl)phenyl)-1,3,4-oxadiazol-2-yl)morpholine was prepared according to TP2 using 2-(4-(trifluoromethyl)phenyl)-1,3,4-oxadiazole $14 \mathrm{e}(107 \mathrm{mg}, 0.50 \mathrm{mmol}$ ) and morpholino benzoate $(73 \mathrm{mg}, 0.35 \mathrm{mmol}$ ). Purification via column chromatography (silica gel, ihexane $/$ EtOAc $=3: 1)$ yielded the title compound $16 \mathrm{~d}(73 \mathrm{mg}, 0.25 \mathrm{mmol}, 70 \%)$ as a colorless oil.

${ }^{1} \mathrm{H}-\mathrm{NMR}\left(\mathrm{CDCl}_{3}, 400 \mathrm{MHz}, \mathrm{ppm}\right): \delta=8.01(\mathrm{~d}, J=8.1 \mathrm{~Hz}, 2 \mathrm{H}), 7.70(\mathrm{~d}, J=8.3 \mathrm{~Hz}, 2 \mathrm{H}), 3.84$ (dd, $J=6.0,3.8 \mathrm{~Hz}, 4 \mathrm{H}), 3.61$ (dd, $J=5.8,3.9 \mathrm{~Hz}, 4 \mathrm{H}$ ).

${ }^{13} \mathrm{C}-N M R\left(\mathrm{CDCl}_{3}, 101 \mathrm{MHz}, \mathrm{ppm}\right): \delta=164.3,158.4,132.2(\mathrm{~d}, J=32.8 \mathrm{~Hz}), 127.6,126.0$, 126.0 (d, $J=3.9 \mathrm{~Hz}, 2 \mathrm{C}), 123.7$ (d, $J=272.4 \mathrm{~Hz}$ ), 65.9, 46.2.

MS (70 eV, EI) m/z (\%): 299 (43) [M] ${ }^{+}, 242$ (65), 173 (100), 145 (79), 114 (45).

IR (ATR) $\widetilde{v}\left(\mathbf{c m}^{-1}\right):$ 2921, 2860, 1711, 1693, 1613, 1584, 1563, 1452, 1425, 1323, 1274, 1258 , 1167, 1113, 1070, 1025, 1016, 913, 847, 730, 714, 685.

HRMS (EI) calculated for $\mathrm{C}_{13} \mathrm{H}_{12} \mathrm{~F}_{3} \mathrm{~N}_{3} \mathrm{O}_{2}{ }^{+}:$299.0882, found 299.0874 [M] ${ }^{+}$.

$N, N$-Diallyl-5-phenyl-1,3,4-oxadiazol-2-amine (16e)

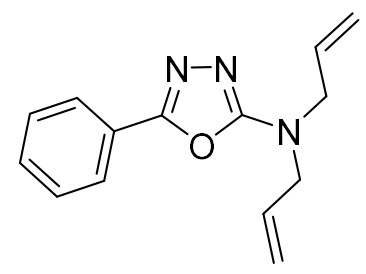

$N, N$-Diallyl-5-phenyl-1,3,4-oxadiazol-2-amine was prepared according to TP2 using 2-phenyl1,3,4-oxadiazole 14a $(73 \mathrm{mg}, 0.50 \mathrm{mmol})$ and $N, N$-diallyl-O-benzoylhydroxylamine $(76 \mathrm{mg}$, 
$0.35 \mathrm{mmol}$ ). Purification via column chromatography (silica gel, ihexane / EtOAc $=3: 1$ ) yielded the title compound $16 \mathrm{e}$ (78 $\mathrm{mg}, 0.32 \mathrm{mmol}, 92 \%)$ as a colorless oil.

${ }^{1} \mathrm{H}-\mathrm{NMR}\left(\mathrm{CDCl}_{3}, 400 \mathrm{MHz}, \mathrm{ppm}\right): \delta=7.89(\mathrm{dt}, J=5.9,3.6 \mathrm{~Hz}, 2 \mathrm{H}), 7.48-7.38(\mathrm{~m}, 3 \mathrm{H}), 5.87$ (ddt, $J=17.2,10.1,6.0 \mathrm{~Hz}, 2 \mathrm{H}), 5.33-5.20(\mathrm{~m}, 4 \mathrm{H}), 4.09(\mathrm{dt}, J=6.0,1.5 \mathrm{~Hz}, 4 \mathrm{H}$ ).

${ }^{13} \mathrm{C}-\mathrm{NMR}\left(\mathrm{CDCl}_{3}, 101 \mathrm{MHz}, \mathrm{ppm}\right): \delta=164.0,159.1,132.2(2 \mathrm{C}), 130.4(2 \mathrm{C}), 128.8(2 \mathrm{C}), 125.7$ (2C), 124.7, $118.5(2 \mathrm{C}), 50.6(2 \mathrm{C})$.

MS (70 eV, El) m/z (\%): 241 (6) [M] ${ }^{+}, 200$ (16), 174 (29), 105 (82), 77 (100).

IR (ATR) $\widetilde{v}\left(\mathbf{c m}^{-1}\right):$ 2920, 2359, 1712, 1643, 1613, 1604, 1583, 1556, 1491, 1449, 1418, 1355 , $1281,1255,1221,1179,1113,1057,1024,1004,991,964,925,888,769,728,689,683$, 668.

HRMS (EI) calculated for $\mathrm{C}_{14} \mathrm{H}_{15} \mathrm{~N}_{3} \mathrm{O}^{+*}: 241.1215$, found 241.1213 [M] ${ }^{+}$.

\section{2-(Azepan-1-yl)-5-(4-methoxyphenyl)-1,3,4-oxadiazole (16f)}

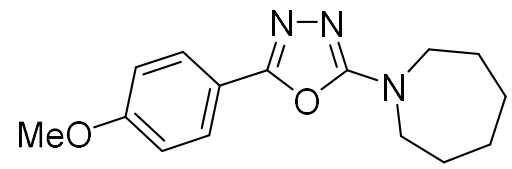

2-(Azepan-1-yl)-5-(4-methoxyphenyl)-1,3,4-oxadiazole was prepared according to TP2 using 2-(4-methoxyphenyl)-1,3,4-oxadiazole $14 \mathrm{f}$ (88 $\mathrm{mg}, 0.50 \mathrm{mmol}$ ) and azepan-1-yl benzoate $(77 \mathrm{mg}, 0.35 \mathrm{mmol})$. Purification via column chromatography (silica gel, ihexane $/ \mathrm{EtOAc}=$ $3: 1)$ yielded the title compound $16 f(75 \mathrm{mg}, 0.27 \mathrm{mmol}, 78 \%)$ as a colorless oil.

${ }^{1} \mathrm{H}-\mathrm{NMR}\left(\mathrm{CDCl}_{3}, 400 \mathrm{MHz}, \mathrm{ppm}\right): \delta=7.84(\mathrm{~d}, J=8.3 \mathrm{~Hz}, 2 \mathrm{H}), 6.95(\mathrm{~d}, J=8.2 \mathrm{~Hz}, 2 \mathrm{H}), 3.85$ (s, 3H), $3.74-3.55(\mathrm{~m}, 3 \mathrm{H}), 1.80(\mathrm{~d}, J=25.9 \mathrm{~Hz}, 5 \mathrm{H}), 1.62(\mathrm{p}, J=2.6 \mathrm{~Hz}, 4 \mathrm{H})$.

${ }^{13} \mathrm{C}-N M R\left(\mathrm{CDCl}_{3}, 101 \mathrm{MHz}, \mathrm{ppm}\right): \delta=161.1(2 \mathrm{C}), 127.3(2 \mathrm{C}), 117.6,114.2(3 \mathrm{C}), 55.4,48.4$ (2C), $28.2(2 \mathrm{C}), 27.6(2 \mathrm{C})$.

MS (70 eV, El) m/z (\%): $273(20)\left[\mathrm{M}^{+}, 175\right.$ (100), 133 (19), 98 (6).

IR (ATR) $\widetilde{v}\left(\mathbf{c m}^{-1}\right):$ 2928, 2856, 1710, 1624, 1607, 1583, 1562, 1502, 1463, 1443, 1425, 1378 , $1360,1301,1280,1252,1219,1172,1113,1103,1059,1025,996,884,836,812,798,735$, $721,683$.

HRMS (EI) calculated for $\mathrm{C}_{15} \mathrm{H}_{19} \mathrm{~N}_{3} \mathrm{O}_{2}{ }^{+*}: 273.1477$, found $273.1480[\mathrm{M}]^{+}$. 
<smiles>c1ccc(-c2nnc(N3CCN(c4ncccn4)CC3)o2)cc1</smiles>

2-Phenyl-5-(4-(pyrimidin-2-yl)piperazin-1-yl)-1,3,4-oxadiazole was prepared according to TP2 using 2-phenyl-1,3,4-oxadiazole 14a (73 mg, $0.50 \mathrm{mmol}$ ) and 4-(pyrimidin-2-yl)piperazin-1-yl benzoate $(100 \mathrm{mg}, 0.35 \mathrm{mmol})$. Purification via column chromatography (silica gel, ihexane / EtOAc $=3: 1)$ yielded the title compound $16 \mathrm{~g}(101 \mathrm{mg}, 0.33 \mathrm{mmol}, 94 \%)$ as a colorless oil.

${ }^{1} \mathrm{H}-\mathrm{NMR}\left(\mathrm{CDCl}_{3}, 400 \mathrm{MHz}, \mathrm{ppm}\right): \delta=8.30(\mathrm{~d}, \mathrm{~J}=4.7 \mathrm{~Hz}, 2 \mathrm{H}), 7.94-7.85(\mathrm{~m}, 2 \mathrm{H}), 7.46-$ $7.36(\mathrm{~m}, 3 \mathrm{H}), 6.52(\mathrm{t}, J=4.7 \mathrm{~Hz}, 1 \mathrm{H}), 4.02-3.89(\mathrm{~m}, 4 \mathrm{H}), 3.67-3.55(\mathrm{~m}, 4 \mathrm{H})$.

${ }^{13} \mathrm{C}-N M R\left(\mathrm{CDCl}_{3}, 101 \mathrm{MHz}, \mathrm{ppm}\right): \delta=164.1,161.5,159.5,157.8(2 \mathrm{C}), 130.6,128.9(2 \mathrm{C})$, $125.8(2 \mathrm{C}), 124.4,110.6,46.0(2 \mathrm{C}), 42.8(2 \mathrm{C})$.

MS (70 eV, El) $m / \mathbf{z}(\%): 308(21)\left[\mathrm{M}^{+}, 146(39), 134\right.$ (100), 122 (75).

IR (ATR) $\tilde{v}\left(\mathbf{c m}^{-1}\right):$ 2359, 2342, 2338, 1738, 1733, 1708, 1605, 1580, 1552, 1464, 1456, 1446 , $1436,1419,1354,1247,1219,949,725,694,684,668$.

HRMS (EI) calculated for $\mathrm{C}_{16} \mathrm{H}_{16} \mathrm{~N}_{6} \mathrm{O}^{+*}: 308.1386$, found 308.1379 [M] $]^{+}$.

Ethyl 1-(5-(4-chlorophenyl)-1,3,4-oxadiazol-2-yl)piperidine-3-carboxylate (16h)<smiles>CCOC(=O)C1CCCN(c2nnc(-c3ccc(Cl)cc3)o2)C1</smiles>

Ethyl 1-(5-(4-chlorophenyl)-1,3,4-oxadiazol-2-yl)piperidine-3-carboxylate was prepared according to TP2 using 2-(4-chlorophenyl)-1,3,4-oxadiazole 14c (90 mg, $0.50 \mathrm{mmol}$ ) and ethyl 1-(benzoyloxy)-piperidine-3-carboxylate $(97 \mathrm{mg}, \quad 0.35 \mathrm{mmol})$. Purification via column chromatography (silica gel, ihexane / EtOAc $=3: 1)$ yielded the title compound $16 \mathrm{~h}(110 \mathrm{mg}$, $0.33 \mathrm{mmol}, 94 \%)$ as a colorless oil.

${ }^{1} \mathrm{H}-\mathrm{NMR}\left(\mathrm{CDCl}_{3}, 400 \mathrm{MHz}, \mathrm{ppm}\right): \delta=7.83(\mathrm{~d}, \mathrm{~J}=8.6 \mathrm{~Hz}, 2 \mathrm{H}), 7.45-7.36(\mathrm{~m}, 2 \mathrm{H}), 4.20-$ $4.08(\mathrm{~m}, 3 \mathrm{H}), 3.91(\mathrm{dt}, J=12.8,4.0 \mathrm{~Hz}, 1 \mathrm{H}), 3.36(\mathrm{dd}, J=13.1,10.0 \mathrm{~Hz}, 1 \mathrm{H}), 3.18$ (ddd, $J=$ $13.5,10.5,3.3 \mathrm{~Hz}, 1 \mathrm{H}), 2.64(\mathrm{ddd}, J=10.1,6.1,4.0 \mathrm{~Hz}, 1 \mathrm{H}), 2.18-2.07(\mathrm{~m}, 1 \mathrm{H}), 1.90-1.78$ $(\mathrm{m}, 1 \mathrm{H}), 1.78-1.59(\mathrm{~m}, 2 \mathrm{H}), 1.25(\mathrm{t}, J=7.1 \mathrm{~Hz}, 3 \mathrm{H})$. 
${ }^{13} \mathrm{C}-N M R\left(\mathrm{CDCl}_{3}, 101 \mathrm{MHz}, \mathrm{ppm}\right): \delta=172.8,164.1,158.5,136.6,129.3$ (2C), 127.1 (2C), 123.1, 61.0, 48.0, 46.6, 40.7, 26.8, 23.6, 14.3.

MS (70 eV, EI) $\mathbf{m / z}(\%): 335(9)\left[\mathrm{M}^{+}, 262(100), 137\right.$ (22).

IR (ATR) $\widetilde{v}\left(\mathbf{c m}^{-1}\right):$ 2937, 2359, 2334, 1726, 1615, 1600, 1576, 1552, 1485, 1465, 1450, 1396, 1382, 1301, 1265, 1183, 1135, 1092, 1058, 1028, 1013, 928, 908, 857, 833, 727, 681, 668.

HRMS (EI) calculated for $\mathrm{C}_{16} \mathrm{H}_{18} \mathrm{CIN}_{3} \mathrm{O}_{3}{ }^{+*}: 335.1037$, found $335.1032[\mathrm{M}]^{+}$.

8-(5-(4-Methoxyphenyl)-1,3,4-oxadiazol-2-yl)-1,4-dioxa-8-azaspiro[4.5]decane (16i)<smiles>COc1ccc(-c2nnc(N3CCC4(CC3)OCCO4)o2)cc1</smiles>

8-(5-(4-Methoxyphenyl)-1,3,4-oxadiazol-2-yl)-1,4-dioxa-8-azaspiro[4.5]decane was prepared according to TP2 using 2-(4-methoxyphenyl)-1,3,4-oxadiazole $14 \mathrm{f}(88 \mathrm{mg}, 0.50 \mathrm{mmol}$ ) and 1,4-dioxa-8-azaspiro[4.5]decan-8-yl benzoate (92 $\mathrm{mg}, 0.35 \mathrm{mmol})$. Purification via column chromatography (silica gel, ihexane / EtOAc $=3: 1$ ) yielded the title compound 16i $(94 \mathrm{mg}$, $0.30 \mathrm{mmol}, 85 \%$ ) as a colorless oil.

${ }^{1} \mathrm{H}-\mathrm{NMR}\left(\mathrm{CDCl}_{3}, 400 \mathrm{MHz}, \mathrm{ppm}\right): \delta=7.83(\mathrm{~s}, 2 \mathrm{H}), 7.00-6.82(\mathrm{~m}, 2 \mathrm{H}), 3.96(\mathrm{~s}, 4 \mathrm{H}), 3.80(\mathrm{~s}$, $3 \mathrm{H}), 3.72(\mathrm{~s}, 4 \mathrm{H}), 1.80(\mathrm{~s}, 4 \mathrm{H})$.

${ }^{13} \mathrm{C}$-NMR ( $\left.\mathrm{CDCl}_{3}, 101 \mathrm{MHz}, \mathrm{ppm}\right): \delta=161.4(2 \mathrm{C}), 127.5,114.3$ (4C), 106.3, 64.5 (4C), 55.4, 44.6, $34.1(2 \mathrm{C})$.

MS (70 eV, EI) m/z (\%): 317 (100) [M] ${ }^{+}, 203$ (15), 135 (66), 57 (27).

IR (ATR) $\widetilde{v}\left(\mathbf{c m}^{-1}\right):$ 2963, 2877, 1708, 1625, 1621, 1608, 1582, 1562, 1503, 1464, 1443, 1425 , 1403, 1365, 1306, 1256, 1174, 1148, 1114, 1080, 1025, 945, 925, 837, 719.

HRMS (El) calculated for $\mathrm{C}_{16} \mathrm{H}_{19} \mathrm{~N}_{3} \mathrm{O}_{4}{ }^{++}: 317.1376$, found 317.1371 [M] $]^{+}$. 

(16j)<smiles>CN(C)c1ccc(-c2nnc(N3CCC4(CC3)OCCO4)o2)cc1</smiles>

4-(5-(1,4-Dioxa-8-azaspiro[4.5]decan-8-yl)-1,3,4-oxadiazol-2-yl)- $N, N$-dimethylaniline was prepared according to TP2 using N,N-dimethyl-4-(1,3,4-oxadiazol-2-yl)aniline $\mathbf{1 4 g}$ (95 mg, $0.50 \mathrm{mmol}$ ) and 1,4-dioxa-8-azaspiro[4.5]decan-8-yl benzoate (92 $\mathrm{mg}, \quad 0.35 \mathrm{mmol})$. Purification via column chromatography ( silica gel, ihexane $/$ EtOAc $=3: 1$ ) yielded the title compound 16j (91 mg, $0.28 \mathrm{mmol}, 79 \%$ ) as a colorless oil.

${ }^{1} \mathrm{H}-\mathrm{NMR}\left(\mathrm{CDCl}_{3}, 400 \mathrm{MHz}, \mathrm{ppm}\right): \delta=7.80-7.62(\mathrm{~m}, 2 \mathrm{H}), 6.73-6.58(\mathrm{~m}, 2 \mathrm{H}), 3.96(\mathrm{~s}, 4 \mathrm{H})$, $3.73-3.57(\mathrm{~m}, 4 \mathrm{H}), 2.98(\mathrm{~s}, 6 \mathrm{H}), 1.93-1.72(\mathrm{~m}, 4 \mathrm{H})$.

${ }^{13} \mathrm{C}-N M R\left(\mathrm{CDCl}_{3}, 101 \mathrm{MHz}, \mathrm{ppm}\right): \delta=163.4,160.1,151.6,127.1(2 \mathrm{C}), 111.9,111.6(2 \mathrm{C})$, $106.5,64.5(2 \mathrm{C}), 44.8(2 \mathrm{C}), 40.1(2 \mathrm{C}), 34.1(2 \mathrm{C})$.

MS (70 eV, El) m/z (\%): 330 (100) [M] ${ }^{+}, 216$ (11), 96 (22), 57 (6).

IR (ATR) $\widetilde{v}\left(\mathbf{c m}^{-1}\right):$ 2958, 2929, 2884, 1603, 1571, 1555, 1509, 1466, 1445, 1432, 1363, 1282, 1262, 1230, 1191, 1170, 1147, 1111, 1079, 1034, 1022, 944, 924, 886, 820, 737.

HRMS (EI) calculated for $\mathrm{C}_{17} \mathrm{H}_{22} \mathrm{~N}_{4} \mathrm{O}_{3}{ }^{++}: 330.1692$, found 330.1695 [M] ${ }^{+}$.

N-(1-(5-Phenyl-1,3,4-oxadiazol-2-yl)piperidin-3-yl)cyclopropanecarboxamide (16k)<smiles>O=C(NC1CCCN(c2nnc(-c3ccccc3)o2)C1)C1CC1</smiles>

$\mathrm{N}$-(1-(5-Phenyl-1,3,4-oxadiazol-2-yl)piperidin-3-yl)cyclopropanecarboxamide was prepared according to TP2 using 2-phenyl-1,3,4-oxadiazole $14 \mathrm{a} \quad(73 \mathrm{mg}, 0.50 \mathrm{mmol}$ ) and 3-(cyclopropanecarboxamido)piperidin-1-yl benzoate (101 mg, $0.35 \mathrm{mmol}$ ). Purification via column chromatography (silica gel, ihexane $/$ EtOAc $=3: 1$ ) yielded the title compound 16k (86 mg, $0.28 \mathrm{mmol}, 79 \%$ ) as a colorless oil. 
${ }^{1} \mathrm{H}-\mathrm{NMR}\left(\mathrm{CDCl}_{3}, 400 \mathrm{MHz}, \mathrm{ppm}\right): \delta=7.94-7.80(\mathrm{~m}, 2 \mathrm{H}), 7.42(\mathrm{~d}, J=6.5 \mathrm{~Hz}, 3 \mathrm{H}), 6.52(\mathrm{~d}, J$ $=7.4 \mathrm{~Hz}, 1 \mathrm{H}), 4.19-4.08(\mathrm{~m}, 1 \mathrm{H}), 3.86-3.73(\mathrm{~m}, 1 \mathrm{H}), 3.62-3.43(\mathrm{~m}, 3 \mathrm{H}), 1.96-1.81(\mathrm{~m}$, $2 \mathrm{H}), 1.73(\mathrm{dd}, J=10.6,6.7 \mathrm{~Hz}, 2 \mathrm{H}), 1.41(\mathrm{tt}, J=8.1,4.5 \mathrm{~Hz}, 1 \mathrm{H}), 0.98-0.59(\mathrm{~m}, 4 \mathrm{H})$.

${ }^{13} \mathrm{C}$-NMR ( $\left.\mathrm{CDCl}_{3}, 101 \mathrm{MHz}, \mathrm{ppm}\right): \delta=173.5,171.3,130.9(2 \mathrm{C}), 129.0(2 \mathrm{C}), 126.0(2 \mathrm{C}), 124.2$, $50.9,46.7,44.8,29.1,21.8,14.7,7.4,7.4$.

MS (70 eV, El) m/z (\%): $312(1)\left[\mathrm{M}^{+}, 227\right.$ (100), 145 (45), 77 (43).

IR (ATR) $\widetilde{v}\left(\mathbf{c m}^{-1}\right):$ 2929, 2856, 2356, 2334, 1642, 1626, 1585, 1557, 1450, 1270, 1247, 1031, 930, 772, 729, 690 .

HRMS (EI) calculated for $\mathrm{C}_{17} \mathrm{H}_{20} \mathrm{~N}_{4} \mathrm{O}_{2}{ }^{+*}: 312.1586$, found $312.1583[\mathrm{M}]^{+}$.

\section{5-lodo-1-propyl-1H-1,2,4-triazole (18a)}

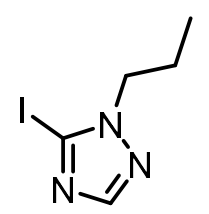

5-lodo-1-propyl-1H-1,2,4-triazole was prepared according to TP3 using iodine (152mg, $0.60 \mathrm{mmol}$ ). Purification via column chromatography (silica gel, ihexane $/$ EtOAc $=4: 1+5 \%$ triethylamine) yielded the title compound $18 \mathrm{a}(96 \mathrm{mg}, 0.41 \mathrm{mmol}, 81 \%)$ as an orange oil.

${ }^{1} \mathrm{H}-\mathrm{NMR}\left(\mathrm{CDCl}_{3}, 400 \mathrm{MHz}, \mathrm{ppm}\right): \delta=7.94(\mathrm{~s}, 1 \mathrm{H}), 4.19-4.11(\mathrm{~m}, 5 \mathrm{H}), 1.90(\mathrm{~h}, J=7.4 \mathrm{~Hz}$, $2 \mathrm{H}), 0.95$ (t, $J=7.4 \mathrm{~Hz}, 3 \mathrm{H})$.

${ }^{13} \mathrm{C}-\mathrm{NMR}\left(\mathrm{CDCl}_{3}, 101 \mathrm{MHz}, \mathrm{ppm}\right): \delta=154.5,99.7,52.5,23.3,11.1$.

MS (70 eV, El) m/z (\%): [M] 237 (1) [M] $]^{+}, 209$ (25), 208 (12), 196 (27), 195 (100), $168(16)$, 110 (68), 55 (18).

IR (ATR) $\widetilde{v}\left(\mathbf{c m}^{-1}\right):$ 3448, 3114, 2966, 2935, 2877, 2626, 2362, 1740, 1636, 1470, 1416, 1385, 1346, 1315, 1266, 1206, 1168, 1116, 1088, 1022, 966, 899, 875, 801, 747, 683.

HRMS (EI) calculated for $\mathrm{C}_{5} \mathrm{H}_{8} \mathrm{IN}_{3}{ }^{+}: 236.9757$, found $236.9758[\mathrm{M}]^{+}$. 
<smiles>C=CCc1ncnn1CCC</smiles>

5-Allyl-1-propyl-1H-1,2,4-triazole was prepared according to TP3. After the metalation a solution of $\mathrm{CuCN} \cdot 2 \mathrm{LiCl}(0.04 \mathrm{~mL}, 0.04 \mathrm{mmol}, 20 \mathrm{~mol} \%, 1 \mathrm{M}$ in THF) and allyl bromide $(0.07 \mathrm{~mL}, 0.60 \mathrm{mmol})$ were added. Purification via column chromatography (silica gel, ihexane / EtOAc $=4: 1+5 \%$ triethylamine $)$ yielded the title compound $18 \mathrm{~b}(63 \mathrm{mg}, 0.42 \mathrm{mmol}, 83 \%)$ as a pale-yellow solid.

${ }^{1} \mathrm{H}-\mathrm{NMR}\left(\mathrm{CDCl}_{3}, 400 \mathrm{MHz}, \mathrm{ppm}\right): \delta=7.83(\mathrm{~s}, 1 \mathrm{H}), 5.96$ (ddt, $J=16.6,10.1,6.3 \mathrm{~Hz}, 1 \mathrm{H}$ ), $5.23-5.10(\mathrm{~m}, 2 \mathrm{H}), 4.05-3.98(\mathrm{~m}, 2 \mathrm{H}), 3.56(\mathrm{~d}, J=6.3 \mathrm{~Hz}, 2 \mathrm{H}), 1.87(\mathrm{~h}, J=7.4 \mathrm{~Hz}, 2 \mathrm{H})$, $0.92(\mathrm{t}, J=7.4 \mathrm{~Hz}, 3 \mathrm{H})$.

${ }^{13} \mathrm{C}-\mathrm{NMR}\left(\mathrm{CDCl}_{3}, 101 \mathrm{MHz}, \mathrm{ppm}\right): \delta=153.0,150.4,131.9,118.3,50.0,30.6,23.3,11.2$.

MS (70 eV, El) m/z (\%): 151 (17) [M] ${ }^{+}, 150$ (100), 136 (14), 123 (40), 122 (79), 110 (34), 108 (80), 84 (26), 83 (16), 81 (11), 68 (11).

IR (ATR) $\widetilde{v}\left(\mathbf{c m}^{-1}\right):$ 3426, 3084, 2968, 2936, 2878, 2361, 1740, 1642, 1511, 1483, 1427, 1401 , $1348,1275,1185,1152,1090,1047,994,920,877,811,748,688,678$.

HRMS (EI) calculated for $\mathrm{C}_{8} \mathrm{H}_{13} \mathrm{~N}_{3}{ }^{+}:$: 151.1104, found $151.1101[\mathrm{M}]^{+}$.

\section{5-(Cyclohex-2-en-1-yl)-1-propyl-1H-1,2,4-triazole (18c)}<smiles>CCCn1ncnc1C1C=CCCC1</smiles>

5-(Cyclohex-2-en-1-yl)-1-propyl-1H-1,2,4-triazole was prepared according to TP3. After the metalation a solution of CuCN.2LiCl $(0.04 \mathrm{~mL}, 0.04 \mathrm{mmol}, 20 \mathrm{~mol} \%, 1 \mathrm{M}$ in THF) and 3-bromocyclohexene $(0.05 \mathrm{~mL}, 0.60 \mathrm{mmol})$ were added. Purification via column chromatography (silica gel, ihexane $/$ EtOAc $=4: 1+5 \%$ triethylamine) yielded the title compound 18c (84 mg, $0.44 \mathrm{mmol}, 88 \%$ ) as a pale-yellow solid.

${ }^{1} \mathrm{H}-\mathrm{NMR}\left(\mathrm{CDCl}_{3}, 400 \mathrm{MHz}, \mathrm{ppm}\right): \delta=7.81(\mathrm{~s}, 1 \mathrm{H}), 6.01-5.91(\mathrm{~m}, 1 \mathrm{H}), 5.64(\mathrm{dd}, J=10.0$, $2.2 \mathrm{~Hz}, 1 \mathrm{H}), 4.12-3.99(\mathrm{~m}, 2 \mathrm{H}), 3.70-3.62(\mathrm{~m}, 1 \mathrm{H}), 2.25-1.97(\mathrm{~m}, 3 \mathrm{H}), 1.96-1.76(\mathrm{~m}$, $4 \mathrm{H}), 1.72-1.59(\mathrm{~m}, 1 \mathrm{H}), 0.95(\mathrm{t}, J=7.4 \mathrm{~Hz}, 3 \mathrm{H})$. 
${ }^{13} \mathrm{C}-\mathrm{NMR}\left(\mathrm{CDCl}_{3}, 101 \mathrm{MHz}, \mathrm{ppm}\right): \delta=158.0,150.3,130.2,125.4,49.9,33.3,28.5,24.6,23.6$, 21.1, 11.3.

MS (70 eV, EI) m/z (\%): $192(4)\left[\mathrm{M}^{++}, 191\right.$ (7), 190 (17), 176 (32), 163 (25), 162 (100), 150 (15), 148 (41), 134 (28), 120 (61), 108 (13), 96 (13).

IR (ATR) $\widetilde{v}\left(\mathbf{c m}^{-1}\right):$ 3433, 3027, 2935, 2877, 2361, 1653, 1506, 1470, 1398, 1348, 1273, 1217, 1188, 1133, 1052, 1040, 992, 932, 898, 882, 842, 807, 772, 744, 720, 671.

HRMS (EI) calculated for $\mathrm{C}_{11} \mathrm{H}_{17} \mathrm{~N}_{3}{ }^{+}:$191.1417, found $191.1415[\mathrm{M}]^{+}$.

\section{5-(Cyclohex-2-en-1-yl)-1-benzyl-1H-1,2,4-triazole (18d)}

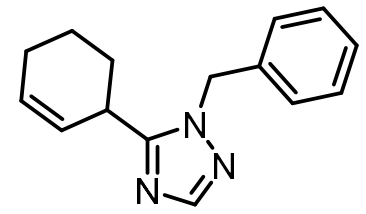

5-(Cyclohex-2-en-1-yl)-1-benzyl-1H-1,2,4-triazole was prepared according to TP3. After the metalation a solution of CuCN.2LiCl $(0.04 \mathrm{~mL}, 0.04 \mathrm{mmol}, 20 \mathrm{~mol} \%, 1 \mathrm{M}$ in THF) and 3-bromocyclohexene $(0.05 \mathrm{~mL}, 0.60 \mathrm{mmol})$ were added. Purification via column chromatography (silica gel, ihexane / EtOAc $=4: 1+5 \%$ triethylamine) yielded the title compound $18 \mathrm{~d}$ (96 $\mathrm{mg}, 0.40 \mathrm{mmol}, 80 \%$ ) as a pale-yellow solid.

${ }^{1} \mathrm{H}-\mathrm{NMR}\left(\mathrm{CDCl}_{3}, 400 \mathrm{MHz}, \mathrm{ppm}\right): \delta=7.88(\mathrm{~s}, 1 \mathrm{H}), 7.43-7.29(\mathrm{~m}, 3 \mathrm{H}), 7.20-7.15(\mathrm{~m}, 2 \mathrm{H})$, 5.95 (ddt, $J=10.0,4.2,2.8 \mathrm{~Hz}, 1 \mathrm{H}$ ), $5.61-5.55$ (m, $1 \mathrm{H}$ ), 5.37 (s, 2H), 3.67 (ddp, J = 8.5, 5.7, $2.8 \mathrm{~Hz}, 1 \mathrm{H}), 2.22-2.00(\mathrm{~m}, 2 \mathrm{H}), 1.95-1.80(\mathrm{~m}, 2 \mathrm{H}), 1.80-1.70(\mathrm{~m}, 1 \mathrm{H}), 1.67-1.54$ $(\mathrm{m}, 1 \mathrm{H})$.

${ }^{13} \mathrm{C}-\mathrm{NMR}\left(\mathrm{CDCl}_{3}, 101 \mathrm{MHz}, \mathrm{ppm}\right): \delta=158.5,150.7,135.7,130.0,128.9,128.2,127.1,125.2$, $52.0,33.4,28.2,24.5,20.9$.

MS (70 eV, El) m/z (\%): 239 (19) [M] ${ }^{+}, 238$ (43), 210 (58), 207 (38), 172 (10), 148 (25), 133 (13), 120 (25), 106 (18), 91 (100).

IR (ATR) $\widetilde{v}\left(\mathbf{c m}^{-1}\right):$ 3031, 2937, 1710, 1676, 1497, 1455, 1397, 1360, 1274, 1222, 1188, 1136, $1089,1030,991,884,803,770,719,694,670$.

HRMS (El) calculated for $\mathrm{C}_{15} \mathrm{H}_{17} \mathrm{~N}_{3}: 239.1422$, found 239.1421 [M]: 


\section{Phenyl(1-propyl-1H-1,2,4-triazol-5-yl)methanol (18e)}<smiles>CCCn1ncnc1C(O)c1ccccc1</smiles>

Phenyl(1-propyl-1H-1,2,4-triazol-5-yl)methanol was prepared according to TP3 using benzaldehyde $(0.06 \mathrm{~mL}, 0.60 \mathrm{mmol})$ as electrophile. Purification via column chromatography (silica gel, ihexane $/$ EtOAc $=1: 1+5 \%$ triethylamine) yielded the title compound $18 \mathrm{e}(103 \mathrm{mg}$, $0.48 \mathrm{mmol}, 95 \%)$ as a colorless oil.

${ }^{1} \mathrm{H}-\mathrm{NMR}\left(\mathrm{CDCl}_{3}, 400 \mathrm{MHz}, \mathrm{ppm}\right): \delta=7.82(\mathrm{~s}, 1 \mathrm{H}), 7.40-7.28(\mathrm{~m}, 5 \mathrm{H}), 6.02(\mathrm{~s}, 1 \mathrm{H}), 3.94-$ $3.88(\mathrm{~m}, 2 \mathrm{H}), 1.62(\mathrm{~h}, J=7.4 \mathrm{~Hz}, 2 \mathrm{H}), 0.78-0.71(\mathrm{~m}, 3 \mathrm{H})$.

${ }^{13} \mathrm{C}-\mathrm{NMR}\left(\mathrm{CDCl}_{3}, 101 \mathrm{MHz}, \mathrm{ppm}\right): \delta=156.1,149.5,139.5,129.0,128.7,126.6,68.6,50.6$, 22.8, 11.0 .

MS (70 eV, EI) $\boldsymbol{m} / \mathbf{z}(\%): 217$ (60) [M] ${ }^{+}, 216$ (12), 184 (13), 175 (17), 174 (40), 170 (100), 158 (41), 143 (48), 140 (18), 118 (26), 116 (14), 110 (20), 105 (28), 98 (26), 77 (14).

IR (ATR) $\widetilde{v}\left(\mathbf{c m}^{-1}\right):$ 3202, 2967, 2936, 2877, 1652, 1603, 1492, 1451, 1385, 1321, 1281, 1228 , 1184, 1155, 1086, 1058, 1027, 984, 900, 877, 849, 800, 749, 718, 697, 674.

HRMS (EI) calculated for $\mathrm{C}_{12} \mathrm{H}_{15} \mathrm{~N}_{3} \mathrm{O}^{+*}: 217.1210$, found $217.1208[\mathrm{M}]^{+}$.

\section{Phenyl(1-benzyl-1H-1,2,4-triazol-5-yl)methanol (18f)}<smiles>OC(c1ccccc1)c1ncnn1Cc1ccccc1</smiles>

Phenyl(1-benzyl-1H-1,2,4-triazol-5-yl)methanol was prepared according to TP2 using benzaldehyde $(0.06 \mathrm{~mL}, 0.60 \mathrm{mmol})$ as electrophile. Purification via column chromatography (silica gel, ihexane $/$ EtOAc $=2: 1+5 \%$ triethylamine $)$ yielded the title compound $18 \mathrm{f}(121 \mathrm{mg}$, $0.46 \mathrm{mmol}, 91 \%)$ as a colorless solid.

${ }^{1} \mathrm{H}-\mathrm{NMR}\left(\mathrm{CDCl}_{3}, 400 \mathrm{MHz}, \mathrm{ppm}\right): \delta=\delta 7.88(\mathrm{~s}, 1 \mathrm{H}), 7.37-7.28(\mathrm{~m}, 5 \mathrm{H}), 7.29-7.23(\mathrm{~m}$, $2 \mathrm{H}), 7.05-6.98(\mathrm{~m}, 2 \mathrm{H}), 6.00(\mathrm{~s}, 2 \mathrm{H}), 5.27(\mathrm{~d}, J=15.2 \mathrm{~Hz}, 1 \mathrm{H}), 5.10(\mathrm{~d}, J=15.2 \mathrm{~Hz}, 1 \mathrm{H})$, 2.89 (br. s, $1 \mathrm{H})$. 
${ }^{13} \mathrm{C}-\mathrm{NMR}\left(\mathrm{CDCl}_{3}, 101 \mathrm{MHz}, \mathrm{ppm}\right): \delta=156.2,149.4,139.0,134.6,129.1,128.9,128.8,128.5$, $127.8,126.8,68.7,52.9$.

MS (70 eV, El) m/z (\%): 265 (6) [M] ${ }^{+}, 247$ (37), 246 (16), 186 (19), 174 (100), 170 (15), 167 (13), 158 (28), 131 (14), 117 (17), 105 (36), 104 (27), 96 (13), 91 (67), 77 (21).

IR (ATR) $\widetilde{v}\left(\mathbf{c m}^{-1}\right):$ 3064, 2862, 2361, 1602, 1494, 1453, 1436, 1399, 1358, 1334, 1303, 1280, 1264, 1178, 1099,1071, 1052, 1028, 989, 954, 899, 853, 779, 735, 714, 699, 690.

HRMS (EI) calculated for $\mathrm{C}_{16} \mathrm{H}_{15} \mathrm{~N}_{3} \mathrm{O}^{+*}: 265.1210$, found $265.1212[\mathrm{M}]^{+}$.

\section{Phenyl(1-propyl-1H-1,2,4-triazol-5-yl)methanone (18g)}<smiles>CCCn1ncnc1C(=O)c1ccccc1</smiles>

Phenyl(1-propyl-1H-1,2,4-triazol-5-yl)methanone was prepared according to TP2 using benzoyl chloride $(0.07 \mathrm{mg}, 0.60 \mathrm{mmol})$ as electrophile. Purification via column chromatography (silica gel, ihexane $+5 \%$ triethylamine) yielded the title compound $\mathbf{1 8 g}$ (95 $\mathrm{mg}, 0.44 \mathrm{mmol}, 88 \%$ ) as a colorless oil.

${ }^{1} \mathrm{H}-\mathrm{NMR}\left(\mathrm{CDCl}_{3}, 400 \mathrm{MHz}, \mathrm{ppm}\right): \delta=8.33-8.27(\mathrm{~m}, 2 \mathrm{H}), 8.03(\mathrm{~s}, 1 \mathrm{H}), 7.69-7.61(\mathrm{~m}, 1 \mathrm{H})$, $7.57-7.48(\mathrm{~m}, 2 \mathrm{H}), 4.59-4.53(\mathrm{~m}, 2 \mathrm{H}), 1.96(\mathrm{~h}, J=7.4 \mathrm{~Hz}, 2 \mathrm{H}), 0.97(\mathrm{t}, J=7.4 \mathrm{~Hz}, 3 \mathrm{H})$.

${ }^{13} \mathrm{C}-\mathrm{NMR}\left(\mathrm{CDCl}_{3}, 101 \mathrm{MHz}, \mathrm{ppm}\right): \delta=183.7,150.1,149.1,136.1,134.2,131.0,128.7,52.9$, 23.8, 11.1 .

MS (70 eV, El) m/z (\%): 215 (16) [M] ${ }^{+}, 214$ (57), 187 (11), 186 (100), 108 (15), 105 (35), 77 (16).

IR (ATR) $\tilde{v}\left(\mathbf{c m}^{-1}\right):$ 2968, 2878, 1659, 1598, 1579, 1495, 1449, 1431, 1375, 1266, 1212, 1178 , $1144,1063,1002,977,912,807,737,680$.

HRMS (El) calculated for $\mathrm{C}_{12} \mathrm{H}_{13} \mathrm{~N}_{3} \mathrm{O}^{+*}: 215.1053$, found $215.1051[\mathrm{M}]^{+}$. 
<smiles>O=C(c1ccccc1)c1ncnn1Cc1ccccc1</smiles>

(1-Benzyl-1H-1,2,4-triazol-5-yl)(phenyl)methanone was prepared according to TP3 using benzaldehyde $(0.06 \mathrm{~mL}, 0.60 \mathrm{mmol})$ as electrophile. Purification via column chromatography (silica gel, ihexane $/$ EtOAc $=2: 1+5 \%$ triethylamine) yielded the title compound $18 \mathrm{~h}$ (104 mg, $0.40 \mathrm{mmol}, 79 \%$ ) as a colorless solid.

${ }^{1} \mathrm{H}-\mathrm{NMR}\left(\mathrm{CDCl}_{3}, 400 \mathrm{MHz}, \mathrm{ppm}\right): \delta=8.26-8.20(\mathrm{~m}, 2 \mathrm{H}), 7.99(\mathrm{~s}, 1 \mathrm{H}), 7.60-7.53(\mathrm{~m}, 1 \mathrm{H})$, $7.47-7.41(\mathrm{~m}, 2 \mathrm{H}), 7.34-7.20(\mathrm{~m}, 5 \mathrm{H}), 5.74(\mathrm{~s}, 2 \mathrm{H})$.

${ }^{13} \mathrm{C}-\mathrm{NMR}\left(\mathrm{CDCl}_{3}, 101 \mathrm{MHz}, \mathrm{ppm}\right): \delta=183.4,150.4,148.9,135.8,135.5,134.1,131.0,128.8$, $128.5,128.3,128.2,54.5$.

MS (70 eV, El) m/z (\%): 264 (13), $263(79)\left[\mathrm{M}^{+}, 262\right.$ (29), 236 (15), 235 (36), $186(10)$, 160 (12), 158 (52), 157 (37), 133 (18), 131 (100), 116 (13), 105 (75), 104 (66), 91 (23), 77 (44).

IR (ATR) $\widetilde{v}\left(\mathbf{c m}^{-1}\right): 3064,1659,1597,1578,1495,1484,1448,1426,1372,1319,1266,1175$, 1098, 1072, 1029, 1002, 976, 911, 807, 785, 717, 681.

HRMS (EI) calculated for $\mathrm{C}_{16} \mathrm{H}_{13} \mathrm{~N}_{3} \mathrm{O}^{+*}: 263.1053$, found 263.1055 [M] $]^{+}$.

\section{5-(4-Methoxyphenyl)-1-propyl-1H-1,2,4-triazole (18i)}<smiles>CCCn1ncnc1-c1ccc(OC)cc1</smiles>

5-(4-Methoxyphenyl)-1-propyl-1H-1,2,4-triazole was prepared according to TP6 using 4-bromoanisole $(112 \mathrm{mg}, 0.6 \mathrm{mmol})$. Purification via column chromatography (silica gel, ihexane / EtOAc $=4: 1+5 \%$ triethylamine) yielded the title compound $18 \mathrm{i}(99 \mathrm{mg}, 0.46 \mathrm{mmol}$, $91 \%)$ as an orange oil.

${ }^{1} \mathrm{H}-\mathrm{NMR}\left(\mathrm{CDCl}_{3}, 400 \mathrm{MHz}, \mathrm{ppm}\right): \delta=7.95(\mathrm{~s}, 1 \mathrm{H}), 7.59-7.54(\mathrm{~m}, 2 \mathrm{H}), 7.05-7.00(\mathrm{~m}, 2 \mathrm{H})$, $4.20-4.13(\mathrm{~m}, 2 \mathrm{H}), 3.87(\mathrm{~s}, 3 \mathrm{H}), 1.92(\mathrm{~h}, J=7.4 \mathrm{~Hz}, 2 \mathrm{H}), 0.90(\mathrm{t}, J=7.4 \mathrm{~Hz}, 3 \mathrm{H})$.

${ }^{13} \mathrm{C}$-NMR ( $\left.\mathrm{CDCl}_{3}, 101 \mathrm{MHz}, \mathrm{ppm}\right): \delta=161.1,154.5,150.5,130.4,120.3,114.5,55.6,50.9$, 23.5, 11.2 . 
MS (70 eV, EI) m/z (\%): 217 (32) [M]+', 188 (63), 175 (100), 160 (25), 134 (90), 133 (13), 132 (10).

IR (ATR) $\widetilde{v}\left(\mathbf{c m}^{-1}\right):$ 3426, 2965, 2936, 2877, 2839, 2361, 2049, 1740, 1613, 1579, 1541, 1486 , $1461,1440,1384,1297,1277,1250,1177,1163,1111,1085,1031,1017,1003,973,900$, $880,836,798,748,713,669$.

HRMS (EI) calculated for $\mathrm{C}_{12} \mathrm{H}_{15} \mathrm{~N}_{3} \mathrm{O}^{+*}: 217.1210$, found 217.1209 [M] $]^{+}$.

5-(4-Methoxyphenyl)-1-benzyl-1H-1,2,4-triazole (18j)<smiles>COc1ccc(-c2ncnn2Cc2ccccc2)cc1</smiles>

5-(4-Methoxyphenyl)-1-benzyl-1H-1,2,4-triazole was prepared according to TP6 using 4-iodoanisole (140 mg, $0.6 \mathrm{mmol}$ ). Purification via column chromatography (silica gel, ihexane / EtOAc $=4: 1+5 \%$ triethylamine $)$ yielded the title compound $18 \mathrm{j}(103 \mathrm{mg}, 0.39 \mathrm{mmol}, 78 \%)$ as an colorless solid.

${ }^{1} \mathrm{H}-\mathrm{NMR}\left(\mathrm{CDCl}_{3}, 400 \mathrm{MHz}, \mathrm{ppm}\right): \delta=8.00(\mathrm{~s}, 1 \mathrm{H}), 7.52(\mathrm{~d}, \mathrm{~J}=8.8 \mathrm{~Hz}, 2 \mathrm{H}), 7.41-7.28(\mathrm{~m}$, $3 \mathrm{H}), 7.16(\mathrm{~d}, \mathrm{~J}=6.6 \mathrm{~Hz}, 2 \mathrm{H}), 6.96(\mathrm{~d}, \mathrm{~J}=8.8 \mathrm{~Hz}, 2 \mathrm{H}), 5.42(\mathrm{~s}, 2 \mathrm{H}), 3.85(\mathrm{~s}, 3 \mathrm{H})$.

${ }^{13} \mathrm{C}-\mathrm{NMR}\left(\mathrm{CDCl}_{3}, \mathbf{1 0 1} \mathbf{M H z}, \mathrm{ppm}\right): \delta=161.2,155.3,151.3,136.1,130.3,129.1,128.2,127.0$, $120.3,114.4,55.5,52.8$.

MS (70 eV, El) m/z (\%): 265 (100) [M]+, 264 (26), 132 (15), 131 (12), 119 (11), 91 (55).

IR (ATR) $\widetilde{v}\left(\mathbf{c m}^{-1}\right):$ 3032, 2938, 2837, 1612, 1578, 1541, 1486, 1462, 1439, 1381, 1298, 1276 , 1252, 1176, 1124, 1112, 1077, 1037, 1020, 1005, 973, 883, 836, 779, 748, 726, 694, 668.

HRMS (EI) calculated for $\mathrm{C}_{16} \mathrm{H}_{15} \mathrm{~N}_{3} \mathrm{O}^{+*}: 265.1210$, found 265.1210 [M] ${ }^{+}$. 


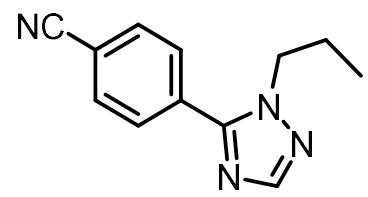

4-(1-Benzyl-1H-1,2,4-triazol-5-yl)benzonitrile was prepared according to TP6 using 4-iodobenzonitrile (137 mg, $0.6 \mathrm{mmol})$. Purification via column chromatography (silica gel, ihexane $/ \mathrm{EtOAc}=4: 1+5 \%$ triethylamine $)$ yielded the title compound $18 \mathrm{k}(100 \mathrm{mg}, 0.47 \mathrm{mmol}$, $94 \%)$ as a colorless solid.

${ }^{1} \mathrm{H}-\mathrm{NMR}\left(\mathrm{CDCl}_{3}, 400 \mathrm{MHz}, \mathrm{ppm}\right): \delta=8.00(\mathrm{~s}, 1 \mathrm{H}), 7.84-7.80(\mathrm{~m}, 2 \mathrm{H}), 7.79-7.75(\mathrm{~m}, 2 \mathrm{H})$, $4.23-4.15$ (m, $2 \mathrm{H}), 1.94$ (h, J = 7.4 Hz, $2 \mathrm{H}), 0.90$ (t, J = $7.4 \mathrm{~Hz}, 3 \mathrm{H})$.

${ }^{13} \mathrm{C}-\mathrm{NMR}\left(\mathrm{CDCl}_{3}, 101 \mathrm{MHz}, \mathrm{ppm}\right): \delta=152.9,151.2,132.8,132.5,129.6,118.1,114.1,51.3$, $23.5,11.1$.

MS (70 eV, El) m/z (\%): $212(11)\left[\mathrm{M}^{+}, 211\right.$ (26), 184 (17), 183 (70), 170 (100), 143 (34), 129 (40).

IR (ATR) $\widetilde{v}\left(\mathbf{c m}^{-1}\right):$ 2968, 2879, 2231, 1614, 1485, 1462, 1406, 1385, 1283, 1283, 1241, 1197, $1182,1164,1111,1010,974,901,845,804,782,747,713,700$.

HRMS (EI) calculated for $\mathrm{C}_{12} \mathrm{H}_{12} \mathrm{~N}_{4}{ }^{++}: 212.1056$, found $212.1055[\mathrm{M}]^{+}$.

\section{4-(1-Propyl-1H-1,2,4-triazol-5-yl)morpholine (18I)}<smiles>CCCn1ncnc1N1CCOCC1</smiles>

4-(1-Propyl-1H-1,2,4-triazol-5-yl)morpholine was prepared according to TP5 using morpholino benzoate (124 mg, $0.6 \mathrm{mmol}$ ). Purification via column chromatography (silica gel, ihexane / EtOAc $=2: 1+5 \%$ triethylamine $)$ yielded the title compound $18 \mathrm{l}(71 \mathrm{mg}, 0.36 \mathrm{mmol}, 72 \%)$ as a pale yellow oil.

${ }^{1} \mathrm{H}-\mathrm{NMR}\left(\mathrm{CDCl}_{3}, 400 \mathrm{MHz}, \mathrm{ppm}\right): \delta=7.67(\mathrm{~s}, 1 \mathrm{H}), 3.94-3.89(\mathrm{~m}, 2 \mathrm{H}), 3.86-3.82(\mathrm{~m}, 4 \mathrm{H})$, $3.17-3.12(\mathrm{~m}, 4 \mathrm{H}), 1.90(\mathrm{~h}, J=7.4 \mathrm{~Hz}, 2 \mathrm{H}), 0.93(\mathrm{t}, J=7.4 \mathrm{~Hz}, 3 \mathrm{H})$.

${ }^{13} \mathrm{C}-\mathrm{NMR}\left(\mathrm{CDCl}_{3}, 101 \mathrm{MHz}, \mathrm{ppm}\right): \delta=158.4,149.2,66.6,51.0,49.4,22.6,11.3$.

MS (70 eV, EI) m/z (\%): 196 (6) [M] ${ }^{+}, 167$ (28), 139 (100), 123 (19), 97 (34). 
IR (ATR) $\widetilde{v}\left(\mathbf{c m}^{-1}\right): 3434,2966,2855,2362,1636,1523,1492,1451,1412,1371,1331,1304$, 1272, 1262, 1200, 1175, 1146, 1115, 1072, 1036, 975, 947, 928, 876, 846, 805, 752, 661.

HRMS (EI) calculated for $\mathrm{C}_{9} \mathrm{H}_{16} \mathrm{~N}_{4} \mathrm{O}^{++}:$196.1319, found 196.1316 [M] ${ }^{+}$.

Ethyl 1-(1-benzyl-1H-1,2,4-triazol-5-yl)piperidine-3-carboxylate (18m)<smiles>CCOC(=O)C1CCCN(c2ncnn2Cc2ccccc2)C1</smiles>

Ethyl 1-(1-benzyl-1H-1,2,4-triazol-5-yl)piperidine-3-carboxylate was prepared according to TP4 using ethyl 1-(benzoyloxy)piperidine-3-carboxylate (166 mg, $0.6 \mathrm{mmol}$ ). Purification via column chromatography (silica gel, ihexane $/$ EtOAc $=2: 1+5 \%$ triethylamine) yielded the title compound $18 \mathrm{~m} \mathrm{(94} \mathrm{mg,} 0.35 \mathrm{mmol}, 70 \%$ ) as a colorless solid.

${ }^{1} \mathrm{H}-\mathrm{NMR}\left(\mathrm{CDCl}_{3}, 400 \mathrm{MHz}, \mathrm{ppm}\right): \delta=7.63(\mathrm{~s}, 1 \mathrm{H}), 7.30-7.21(\mathrm{~m}, 3 \mathrm{H}), 7.19-7.14(\mathrm{~m}, 2 \mathrm{H})$, $5.15(\mathrm{~d}, J=2.3 \mathrm{~Hz}, 2 \mathrm{H}), 4.14-3.90(\mathrm{~m}, 2 \mathrm{H}$ ), 3.30 (ddt, $J=12.2,3.8,1.3 \mathrm{~Hz}, 1 \mathrm{H}$ ), $3.15-$ $3.03(\mathrm{~m}, 2 \mathrm{H}), 2.87$ (ddd, $J=12.2,9.4,3.0 \mathrm{~Hz}, 1 \mathrm{H}), 2.66-2.53(\mathrm{~m}, 1 \mathrm{H}), 1.97-1.75(\mathrm{~m}, 1$ $\mathrm{H}), 1.76-1.67(\mathrm{~m}, 2 \mathrm{H}), 1.15(\mathrm{t}, J=7.1 \mathrm{~Hz}, 4 \mathrm{H})$.

${ }^{13} \mathrm{C}-\mathrm{NMR}\left(\mathrm{CDCl}_{3}, 101 \mathrm{MHz}, \mathrm{ppm}\right): \delta=173.1,159.1,149.6,135.8,128.8,127.9,127.3,60.7$, $52.7,51.3,51.1,41.0,26.2,23.9,14.2$.

MS (70 eV, El) m/z (\%): 314 (11) [M] ${ }^{+}, 313$ (55), 269 (15), 241 (86), 237 (26), 207 (29), 177 (19), 156 (16), 149 (17), 91 (100).

IR (ATR) $\widetilde{v}\left(\mathbf{c m}^{-1}\right):$ 2940, 2855, 1985, 1726, 1636, 1526, 1488, 1454, 1381, 1309, 1273, 1181 , 1128, 1096, 1030, 963, 939, 859, 727, 696, 658.

HRMS (EI) calculated for $\mathrm{C}_{17} \mathrm{H}_{22} \mathrm{~N}_{4} \mathrm{O}_{2}: 314.1743$, found 314.1746 [M]. 
<smiles>CN(c1ncnn1Cc1ccccc1)[C@H]1CC[C@@H](c2ccc(Cl)c(Cl)c2)c2ccccc21</smiles>

1-Benzyl-N-((1S,4S)-4-(3,4-dichlorophenyl)-1,2,3,4-tetrahydronaphthalen-1-yl)- $N$-methyl-1H1,2,4-triazol-5-amine was prepared according to TP4 using O-benzoyl- $N$-((1S,4S)-4-(3,4dichlorophenyl)-1,2,3,4-tetrahydronaphthalen-1-yl)-N-methylhydroxylamine (256 mg, $0.6 \mathrm{mmol}$ ). Purification via column chromatography (silica gel, ihexane $/$ EtOAc $=2: 1+5 \%$ triethylamine) yielded the title compound $18 \mathrm{n}(220 \mathrm{mg}, 0.48 \mathrm{mmol}, 95 \%)$ as a colorless solid. ${ }^{1} \mathrm{H}-\mathrm{NMR}\left(\mathrm{CDCl}_{3}, 400 \mathrm{MHz}, \mathrm{ppm}\right): \delta=7.63(\mathrm{~s}, 1 \mathrm{H}), 7.35-7.15(\mathrm{~m}, 5 \mathrm{H}), 7.15-7.04(\mathrm{~m}, 4 \mathrm{H})$, $6.99(\mathrm{~d}, \mathrm{~J}=2.1 \mathrm{hz}, 1 \mathrm{H}), 6.84(\mathrm{dd}, J=7.0,2.0 \mathrm{~Hz}, 1 \mathrm{H}), 6.71(\mathrm{dd}, J=8.3,2.1 \mathrm{~Hz}, 1 \mathrm{H}), 5.33-$ $5.15(\mathrm{~m}, 2 \mathrm{H}), 4.72(\mathrm{dd}, J=10.4,5.8 \mathrm{~Hz}, 1 \mathrm{H}), 4.03(\mathrm{dd}, J=5.8,3.1 \mathrm{~Hz}, 1 \mathrm{H}), 2.64(\mathrm{~s}, 3 \mathrm{H})$, $2.08-1.53(\mathrm{~m}, 4 \mathrm{H})$.

${ }^{13} \mathrm{C}-\mathrm{NMR}\left(\mathrm{CDCl}_{3}, 101 \mathrm{MHz}, \mathrm{ppm}\right): \delta=159.3,149.3,147.1,138.3,136.3,132.3,130.8,130.7$, $130.1,130.1$, 128.9, 128.0, 128.0, 127.6, 127.5, 127.4, 126.6, 60.5, 52.2, 43.1, 33.6, 29.9, 20.4 .

MS (70 eV, El) m/z (\%): 464 (27) [M] ${ }^{+}, 462$ (42), 447 (22), 277 (36), 275 (58), 188 (56), 187 (33), 160 (61), 158 (100), 129 (60), 91 (94).

IR (ATR) $\widetilde{v}\left(\mathbf{c m}^{-1}\right):$ 2938, 1540, 1488, 1466, 1453, 1417, 1395, 1356, 1317, 1279, 1204, 1131, 1080, 1029, 986, 964, 920, 878, 849, 817, 791, 768, 727, 704, 694, 678, 665.

HRMS (EI) calculated for $\mathrm{C}_{26} \mathrm{H}_{24} \mathrm{Cl}_{2} \mathrm{~N}_{4}{ }^{++}: 462.1373$, found 462.1372 [M] ${ }^{+}$. 


\section{3,5-Di(cyclohex-2-en-1-yl)-1-propyl-1H-1,2,4-triazole (19a)}<smiles>CCCn1nc(C2C=CCCC2)nc1C1C=CCCC1</smiles>

3,5-Di(cyclohex-2-en-1-yl)-1-propyl-1H-1,2,4-triazole was prepared according to TP4. After the metalation a solution of $\mathrm{CuCN} \cdot 2 \mathrm{LiCl}(0.04 \mathrm{~mL}, 0.04 \mathrm{mmol}, 20 \mathrm{~mol} \%, 1 \mathrm{M}$ in THF) and 3-bromocyclohexene $(0.25 \mathrm{~mL}, 2.2 \mathrm{mmol}))$ were added. Purification via column chromatography (silica gel, ihexane $/$ EtOAc $=9: 1+5 \%$ triethylamine) yielded the title compound 19a (99 mg, $0.37 \mathrm{mmol}, 73 \%$ ) as an orange oil.

${ }^{1} \mathrm{H}-\mathrm{NMR}\left(\mathrm{CDCl}_{3}, 400 \mathrm{MHz}, \mathrm{ppm}\right): \delta=5.96-5.80(\mathrm{~m}, 3 \mathrm{H}), 5.67-5.60(\mathrm{~m}, 1 \mathrm{H}), 4.05-3.89$ (m, $2 \mathrm{H}), 3.69-3.49(\mathrm{~m}, 2 \mathrm{H}), 2.22-1.95(\mathrm{~m}, 6 \mathrm{H}), 1.93-1.71(\mathrm{~m}, 6 \mathrm{H}), 1.68-1.56(\mathrm{~m}, 2 \mathrm{H})$, $0.91(\mathrm{t}, J=7.4 \mathrm{~Hz}, 3 \mathrm{H})$.

${ }^{13} \mathrm{C}-\mathrm{NMR}\left(\mathrm{CDCl}_{3}, 101 \mathrm{MHz}, \mathrm{ppm}\right): \delta=165.9,157.9,129.7,128.4,127.8,125.8,49.6,35.7$, $33.8,29.0,28.6,24.9,24.5,23.7,21.5,21.2,11.1$.

MS (70 eV, EI) m/z (\%): 271 (100) [M] ${ }^{+}, 270$ (78), 256 (45), 242 (66), 230 (11), 228 (53), 216 (12), 214 (36), 205 (29), 200 (42), 190 (12), 188 (17), 176 (14), 163 (16), 148 (11), 81 (13), 79 (14).

IR (ATR) $\widetilde{v}\left(\mathbf{c m}^{-1}\right):$ 3026, 2933, 2875, 2836, 2362, 1652, 1497, 1447, 1435, 1361, 1326, 1297 , 1258, 1239, 1218, 1190, 1155, 1134, 1063, 1044, 991, 932, 893, 850, 809, 722, 679.

HRMS (EI) calculated for $\mathrm{C}_{17} \mathrm{H}_{25} \mathrm{~N}_{3}: 271.2048$, found 271.2047 [M] .

\section{3,5-Bis(4-methoxyphenyl)-1-propyl-1H-1,2,4-triazole (19b)}<smiles>CCCn1nc(-c2ccc(OC)cc2)nc1-c1ccc(OC)cc1</smiles>

3,5-Bis(4-methoxyphenyl)-1-propyl-1H-1,2,4-triazole was prepared according to TP4. After the metalation a solution of $\mathrm{ZnCl}_{2}(2.0 \mathrm{~mL}, 2.0 \mathrm{mmol}, 1 \mathrm{M}$ in $\mathrm{THF}), \mathrm{Pd}(\mathrm{OAc})_{2}(5.6 \mathrm{mg}$, $0.025 \mathrm{mmol}, 5 \mathrm{~mol} \%$ ), SPhos (20.5 mg, $0.05 \mathrm{mmol}, 10 \mathrm{~mol} \%$ ) and 4-iodoanisole (515 mg, 2.2 $\mathrm{mmol}$ ) were added. Purification via column chromatography (silica gel, ihexane $/$ EtOAc $=4: 1$ 
$+5 \%$ triethylamine) yielded the title compound $19 \mathrm{~b}(84 \mathrm{mg}, 0.26 \mathrm{mmol}, 52 \%)$ as an orange oil.

${ }^{1} \mathrm{H}-\mathrm{NMR}\left(\mathrm{CDCl}_{3}, 400 \mathrm{MHz}, \mathrm{ppm}\right): \delta=8.12-8.04(\mathrm{~m}, 2 \mathrm{H}), 7.63-7.56(\mathrm{~m}, 2 \mathrm{H}), 7.06-6.99$ (m, 2 H), $6.98-6.93(\mathrm{~m}, 2 \mathrm{H}), 4.19-4.11(\mathrm{~m}, 2 \mathrm{H}), 3.88(\mathrm{~s}, 3 \mathrm{H}), 3.85$ (s, $3 \mathrm{H}), 1.95$ (h, J = $7.4 \mathrm{~Hz}, 2 \mathrm{H}), 0.92(\mathrm{t}, J=7.4 \mathrm{~Hz}, 3 \mathrm{H})$.

${ }^{13} \mathrm{C}-\mathrm{NMR}\left(\mathrm{CDCl}_{3}, 101 \mathrm{MHz}, \mathrm{ppm}\right): \delta=161.1,161.0,160.5,155.5,130.4,127.9,124.2,121.0$, $114.4,114.0,55.6,55.4,50.9,23.7,11.2$.

MS (70 eV, EI) m/z (\%): 323 (100) [M] ${ }^{+}, 295$ (11), 294 (67), 282 (13), 281 (77), 267 (11), 207 (16), 161 (21), 134 (45), 133 (76).

IR (ATR) $\widetilde{v}\left(\mathbf{c m}^{-1}\right):$ 2963, 2936, 2837, 2361, 1739, 1612, 1532, 1465, 1441, 1349, 1294, 1245 , 1169, 1134, 1107, 1029, 981, 902, 836, 797, 764, 736, 692.

HRMS (EI) calculated for $\mathrm{C}_{19} \mathrm{H}_{21} \mathrm{~N}_{3} \mathrm{O}^{+}: 323.1628$, found 323.1627 [M] ${ }^{+}$.

\section{4-(3-(Cyclohex-2-en-1-yl)-1-propyl-1H-1,2,4-triazol-5-yl)morpholine (19c)}<smiles>CCCn1nc(C2C=CCCC2)nc1N1CCOCC1</smiles>

4-(1-Propyl-1H-1,2,4-triazol-5-yl)morpholino 18I (86 mg, $0.44 \mathrm{mmol}, 1.0$ equiv) was dissolved in THF $(1.5 \mathrm{~mL})$ and cooled to $0{ }^{\circ} \mathrm{C}$. TMPMgCl$\cdot \mathrm{LiCl}$ solution $5(0.88 \mathrm{mmol}, 2.0$ equiv) was slowly added to the vigorously stirred solution. After $30 \mathrm{~min}$ a solution of $\mathrm{CuCN} \cdot 2 \mathrm{LiCl}(0.04$ $\mathrm{mL}, 0.04 \mathrm{mmol}, 20 \mathrm{~mol} \%, 1 \mathrm{M}$ in THF) and 3-bromocyclohexene $(0.05 \mathrm{~mL}, 0.60 \mathrm{mmol})$ were added. After stirring for $30 \mathrm{~min}$ the mixture was quenched with sat. aq. $\mathrm{NH}_{4} \mathrm{Cl}$ solution $(15 \mathrm{~mL})$, extracted with DCM $(3 \times 15 \mathrm{~mL})$ and dried over anhydrous $\mathrm{MgSO}_{4}$. Purification via column chromatography (silica gel, ihexane $/$ EtOAc $=4: 1+5 \%$ triethylamine) yielded the title compound 19c (97 mg, $0.35 \mathrm{mmol}, 80 \%$ ) as a colorless oil.

${ }^{1} \mathrm{H}-\mathrm{NMR}\left(\mathrm{CDCl}_{3}, 400 \mathrm{MHz}, \mathrm{ppm}\right): \delta=5.86-5.75(\mathrm{~m}, 2 \mathrm{H}), 3.85-3.79(\mathrm{~m}, 2 \mathrm{H}), 3.79-3.73$ (m, 4 H), 3.42 (ddd, J = 8.7, 5.7, 3.0 Hz, 1 H), $3.10-3.03(m, 4$ H), $2.11-1.94(m, 3$ H), 1.87 - $1.66(\mathrm{~m}, 4 \mathrm{H}), 1.62-1.50(\mathrm{~m}, 1 \mathrm{H}), 0.86(\mathrm{t}, \mathrm{J}=7.4 \mathrm{~Hz}, 3 \mathrm{H})$.

${ }^{13} \mathrm{C}-\mathrm{NMR}\left(\mathrm{CDCl}_{3}, 101 \mathrm{MHz}, \mathrm{ppm}\right): \delta=164.5,158.5,128.4,127.6,66.6,50.9,49.0,36.0,28.7$, 24.9, 22.7, 21.2, 11.1. 
MS (70 eV, EI) m/z (\%): 276 (60) [M] +, 275 (19), 247 (37), 233 (20), 220 (13), 219 (100), 210 (19), 205 (14), 203 (11), 189 (10), 177 (31), 167 (10).

IR (ATR) $\widetilde{v}\left(\mathbf{c m}^{-1}\right):$ 2935, 2858, 1713, 1530, 1497, 1453, 1372, 1261, 1219, 1117, 1072, 1029, 984, 964, 945, 921, 881, 849, 792, 727, 678.

HRMS (EI) calculated for $\mathrm{C}_{15} \mathrm{H}_{24} \mathrm{~N}_{4} \mathrm{O}^{+*}: 276.1945$, found 276.1946 [M] ${ }^{+}$.

\section{4-(5-(4-methoxyphenyl)-1-propyl-1H-1,2,4-triazol-3-yl)morpholine (19d)}<smiles>CCCn1nc(N2CCOCC2)nc1-c1ccc(OC)cc1</smiles>

5-(4-Methoxyphenyl)-1-propyl-1H-1,2,4-triazole $18 \mathrm{i}$ (95 $\mathrm{mg}, 0.44 \mathrm{mmol}, 1.0$ equiv) was dissolved in THF $(1.5 \mathrm{~mL})$ and cooled to $0{ }^{\circ} \mathrm{C}$. TMPZnCl. LiCl solution 7 (0.53 mmol, 1.2 equiv) was slowly added to the vigorously stirred solution. A dry, argon flushed Schlenk-flask equipped with a magnetic stirring bar and a septum was charged with morpholino benzoate (109 mg, $0.53 \mathrm{mmol}, 1.2$ equiv), copper (II) triflate (36 mg, $0.1 \mathrm{mmol}, 20 \mathrm{~mol} \%$ ) and THF $(2.0 \mathrm{~mL})$. After $30 \mathrm{~min}$ the metalated species was slowly added to the vigorously stirred solution. After $2 \mathrm{~h}$ the mixture was quenched with sat. aq. $\mathrm{NH}_{4} \mathrm{Cl}$ solution ( $15 \mathrm{~mL}$ ), extracted with DCM $(3 \times 15 \mathrm{~mL})$ and dried over anhydrous $\mathrm{MgSO}_{4}$. Purification via column chromatography (silica gel, ihexane $/$ EtOAc $=4: 1+5 \%$ triethylamine) yielded the title compound 19d (115 mg, $0.38 \mathrm{mmol}, 87 \%$ ) as a colorless oil.

${ }^{1} \mathrm{H}-\mathrm{NMR}\left(\mathrm{CDCl}_{3}, 400 \mathrm{MHz}, \mathrm{ppm}\right): \delta=7.54-7.47(\mathrm{~m}, 2 \mathrm{H}), 7.02-6.95(\mathrm{~m}, 2 \mathrm{H}), 4.01-3.92$ (m, $2 \mathrm{H}), 3.85(\mathrm{~s}, 3 \mathrm{H}), 3.84-3.79(\mathrm{~m}, 4 \mathrm{H}), 3.48-3.41(\mathrm{~m}, 4 \mathrm{H}), 1.86(\mathrm{~h}, J=7.4 \mathrm{~Hz}, 2 \mathrm{H})$, $0.87(\mathrm{t}, J=7.4 \mathrm{~Hz}, 3 \mathrm{H})$.

${ }^{13} \mathrm{C}-\mathrm{NMR}\left(\mathrm{CDCl}_{3}, 101 \mathrm{MHz}, \mathrm{ppm}\right): \delta=161.0,153.8,142.3,138.1,130.4,114.4,66.7,55.5$, $50.5,47.2,23.4,11.2$.

MS (70 eV, EI) m/z (\%): 303 (14) [M] $]^{+}, 302$ (93), 287 (24), 271 (26), 271 (17), 246 (11), 245 (100), 244 (29), 243 (16), 229 (10), 215 (32), 203 (31), 202 (27), 188 (12), 134 (36).

IR (ATR) $\tilde{v}\left(\mathbf{c m}^{-1}\right):$ 3442, 2964, 2838, 2361, 1614, 1579, 1547, 1527, 1482, 1452, 1411, 1379, 1357, 1331, 1304, 1273, 1250, 1174, 1115, 1073, 1032, 1002, 926, 902, 835, 797, 765, 733, 661.

HRMS (EI) calculated for $\mathrm{C}_{16} \mathrm{H}_{22} \mathrm{~N}_{4} \mathrm{O}_{2}{ }^{+}: 302.1737$, found $302.1736[\mathrm{M}]^{+}$. 
$5 H-[1,2,4]$ Triazolo[5,1-a]isoindole $(20)$<smiles>c1ccc2c(c1)Cn1ncnc1-2</smiles>

A dry, argon flushed Schlenk-flask equipped with a magnetic stirring bar and a septum was charged with 1-(2-lodobenzyl)-1H-1,2,4-triazole 12c (143 mg, $0.5 \mathrm{mmol}, 1.0$ equiv) and THF $(1.5 \mathrm{~mL})$. The mixture was cooled down to $0{ }^{\circ} \mathrm{C}$ and a solution of $\mathrm{TMPZnCl} \cdot \mathrm{LiCl} 7(0.75 \mathrm{mmol}$, 1.5 equiv) was slowly added. After $30 \mathrm{~min}$ a solution of $\mathrm{CuCN} \cdot 2 \mathrm{LiCl}(0.10 \mathrm{~mL}, 0.10 \mathrm{mmol}, 20$ mol\%, $1 \mathrm{M}$ in THF) was added. The mixture was heated to $40{ }^{\circ} \mathrm{C}$ for $18 \mathrm{~h}$. The reaction mixture was quenched with sat. aq. $\mathrm{NH}_{4} \mathrm{Cl}$ solution $(15 \mathrm{~mL})$, extracted with $\mathrm{DCM}(3 \times 15 \mathrm{~mL})$ and dried over anhydrous $\mathrm{MgSO}_{4}$. After filtration and evaporation, the crude product was purified by flash column chromatography (silica gel, ihexane / EtOAc $=4: 1+5 \%$ triethyl-amine) to obtain $5 H$-[1,2,4]triazolo[5,1-a]isoindole $20(0.147 \mathrm{~g}, 0.47 \mathrm{mmol}, 94 \%)$ as a pale yellow solid.

${ }^{1} \mathrm{H}-\mathrm{NMR}\left(\mathrm{CDCl}_{3}, 400 \mathrm{MHz}, \mathrm{ppm}\right): \delta=8.11(\mathrm{~s}, 1 \mathrm{H}), 7.98-7.91(\mathrm{~m}, 1 \mathrm{H}), 7.59-7.46(\mathrm{~m}, 3 \mathrm{H})$, $5.10(\mathrm{~s}, 2 \mathrm{H})$.

${ }^{13} \mathrm{C}-\mathrm{NMR}\left(\mathrm{CDCl}_{3}, 101 \mathrm{MHz}, \mathrm{ppm}\right): \delta=160.2,155.8,142.2,129.7,129.1,127.1,124.1,121.9$, 50.4 .

MS (70 eV, EI) m/z (\%): $157(100)\left[\mathrm{M}^{+}, 130\right.$ (16), 129 (14), 103 (25), 102 (11).

IR (ATR) $\widetilde{v}\left(\mathbf{c m}^{-1}\right):$ 3088, 2954, 1722, 1539, 1506, 1447, 1403, 1317, 1274, 1248, 1215, 1191 , 1175, 1136, 1101, 1011, 969, 941, 918, 895, 883, 816, 769, 730, 699, 689, 679, 655.

HRMS (EI) calculated for $\mathrm{C}_{9} \mathrm{H}_{7} \mathrm{~N}_{3}{ }^{+}:$: 157.0634, found $157.0632[\mathrm{M}]^{+}$. 
NMR Spectra

2-Phenyl-1,3,4-oxadiazole (14a)
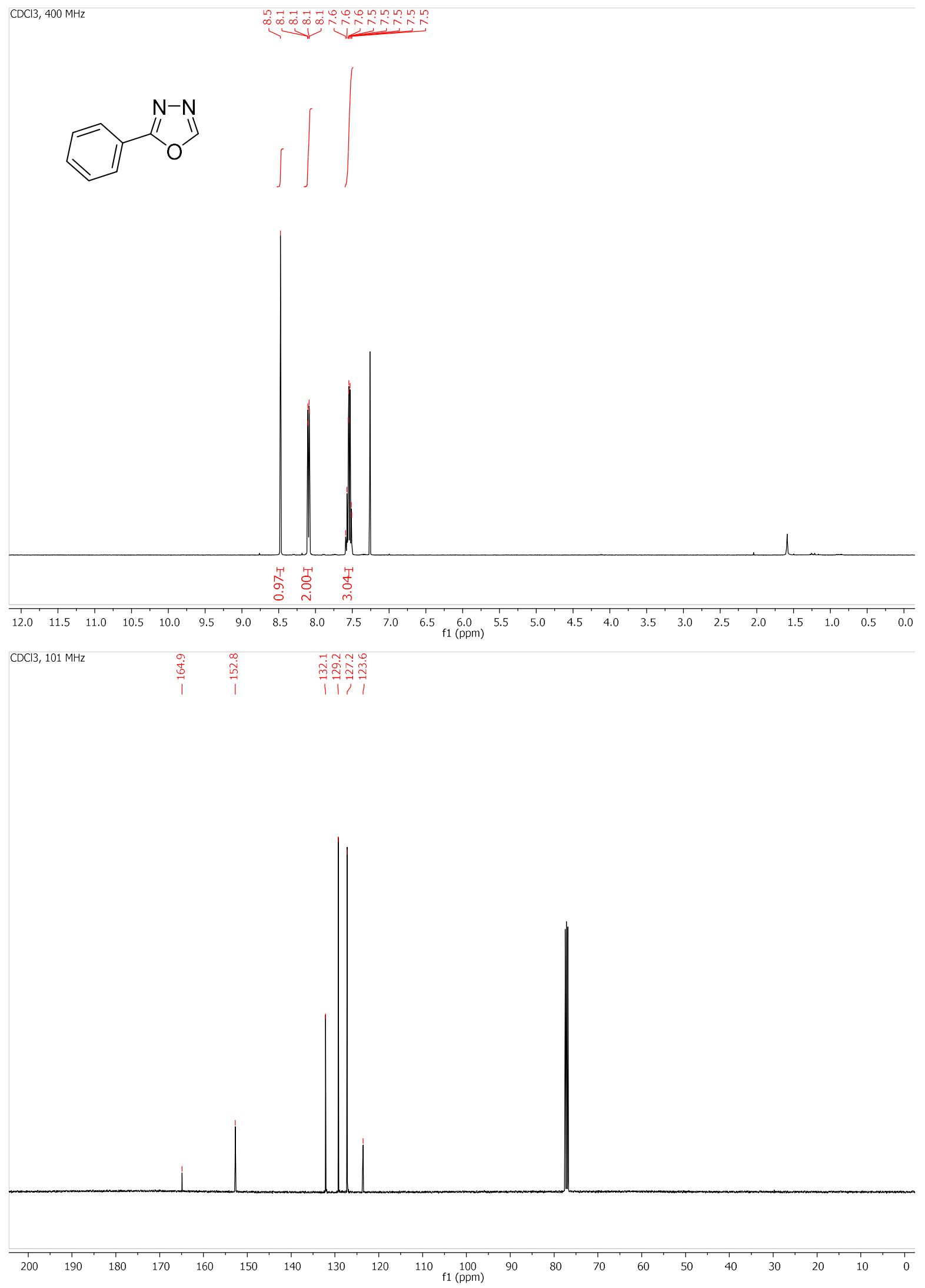

SI 38 
Ethyl 4-(1,3,4-oxadiazol-2-yl)benzoate (14b)
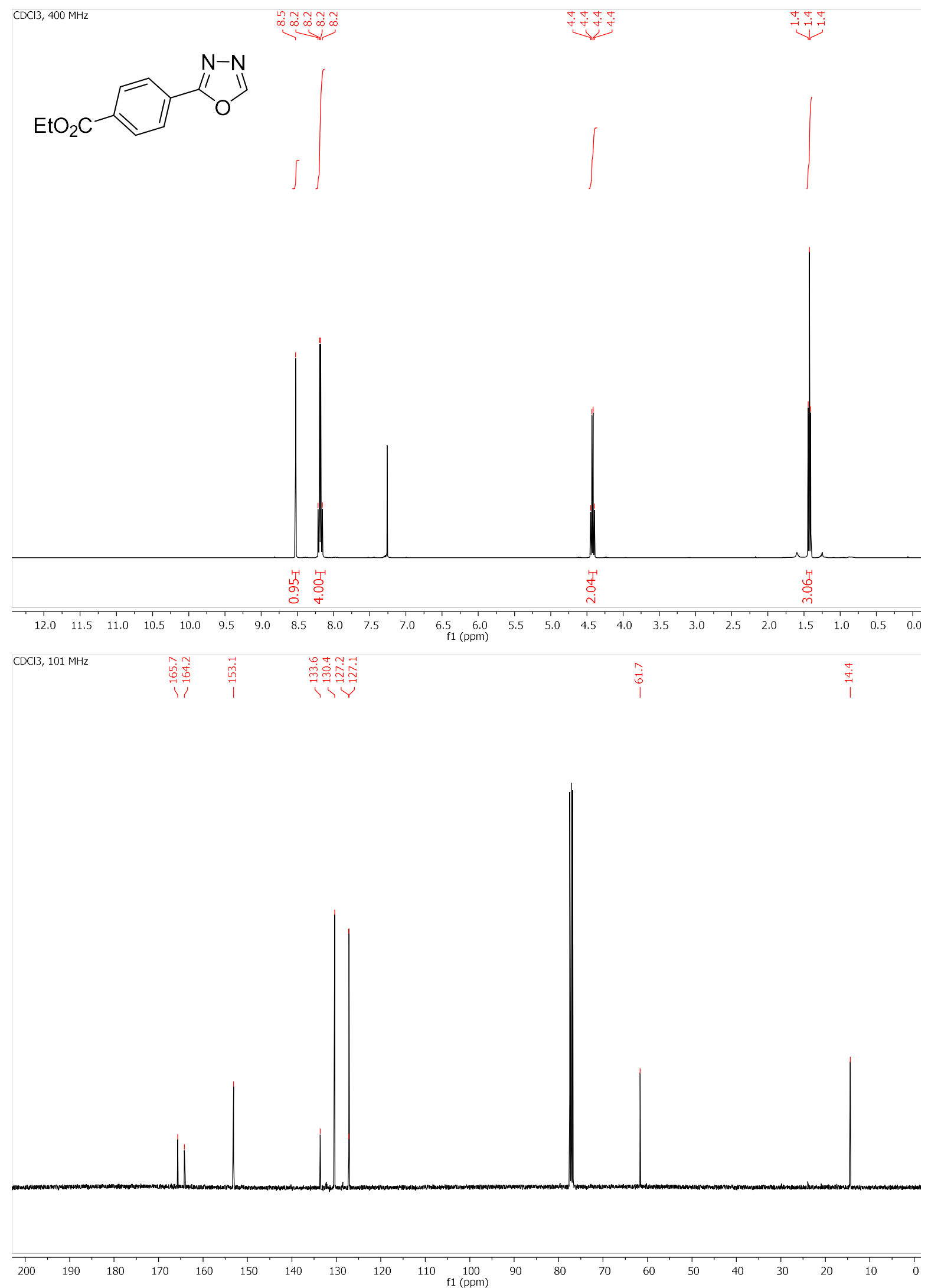
2-(4-Chlorophenyl)-1,3,4-oxadiazole (14c)

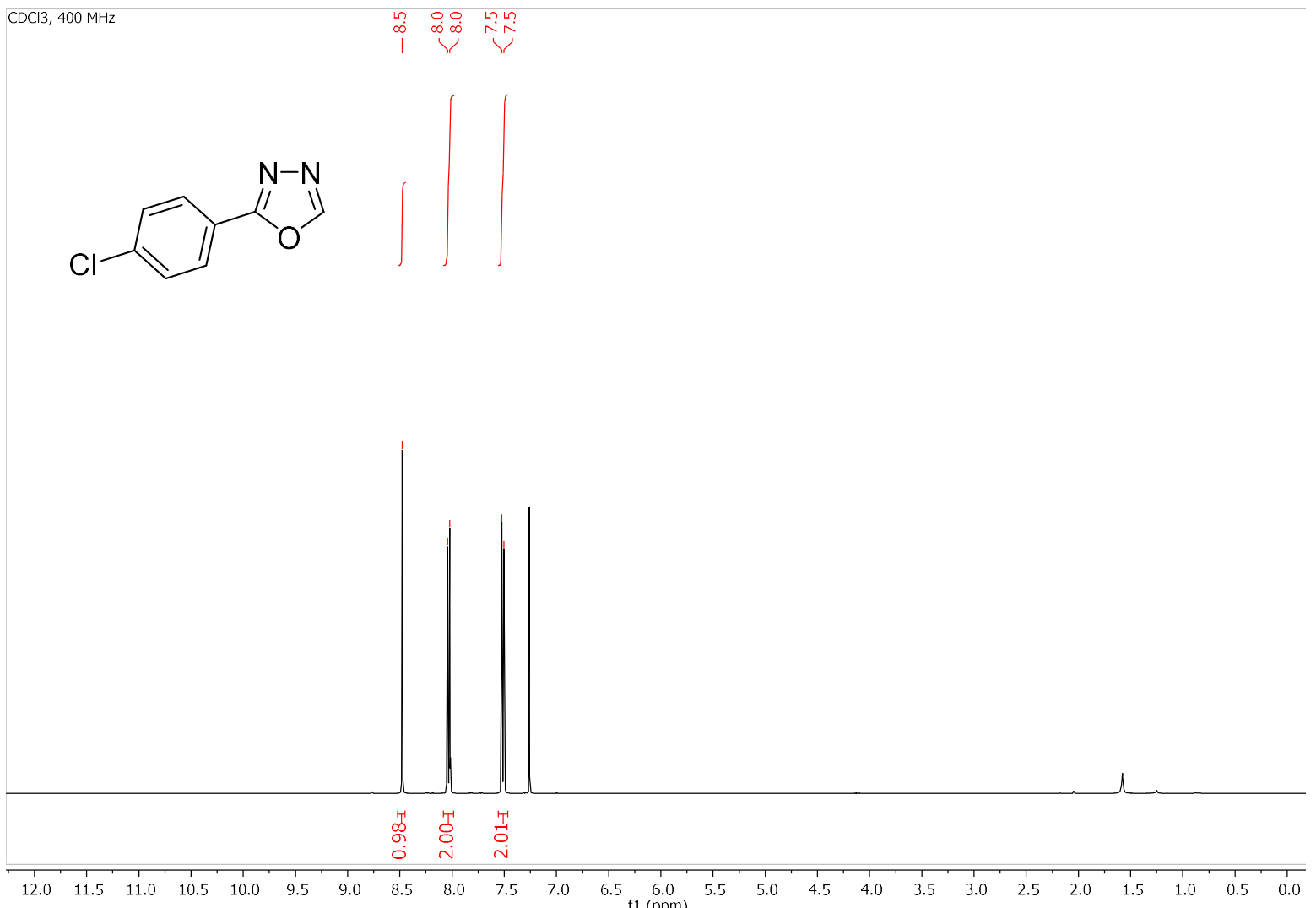

$\mathrm{CDCl}, 101 \mathrm{MHz}$

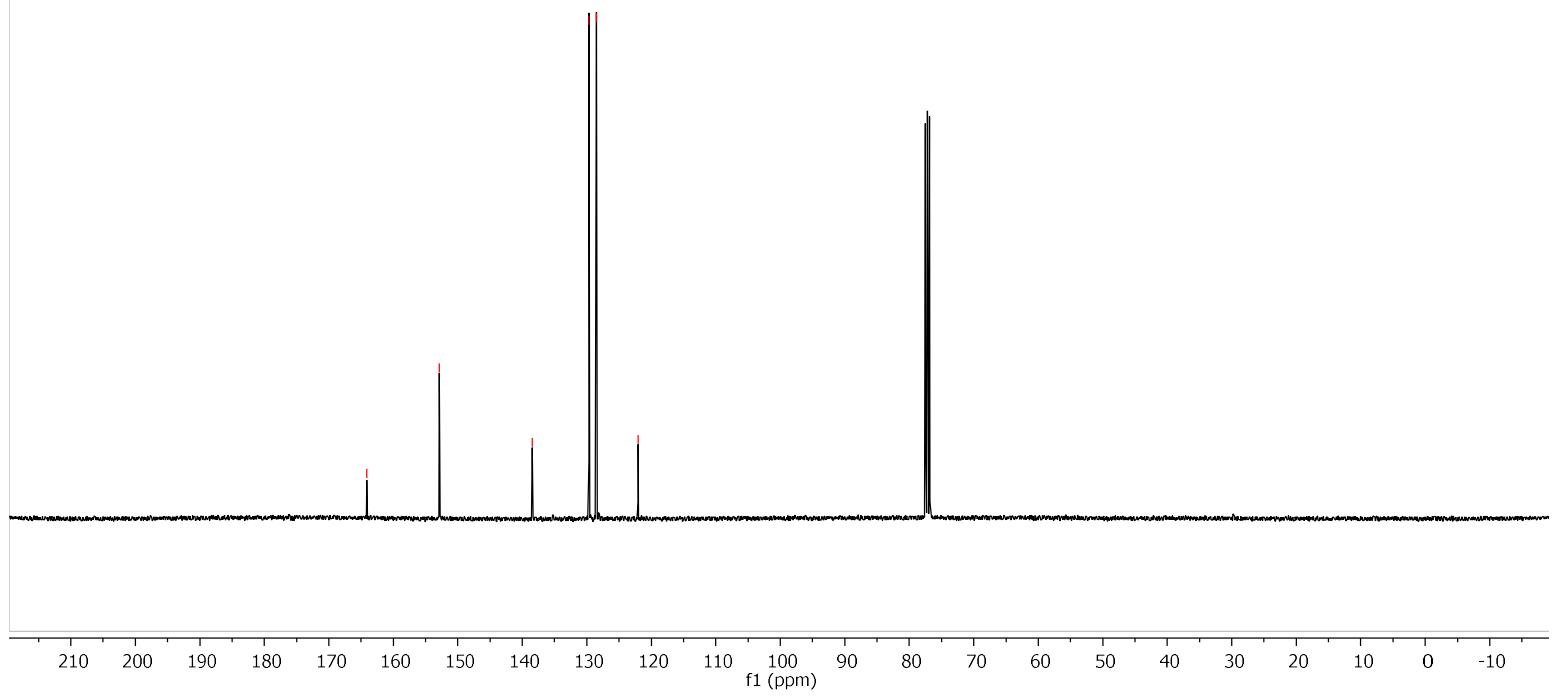


4-(1,3,4-Oxadiazol-2-yl)benzonitrile (14d)

$\mathrm{CDCl} 3,400 \mathrm{MHz}$

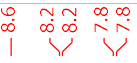

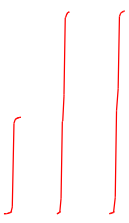

(1)

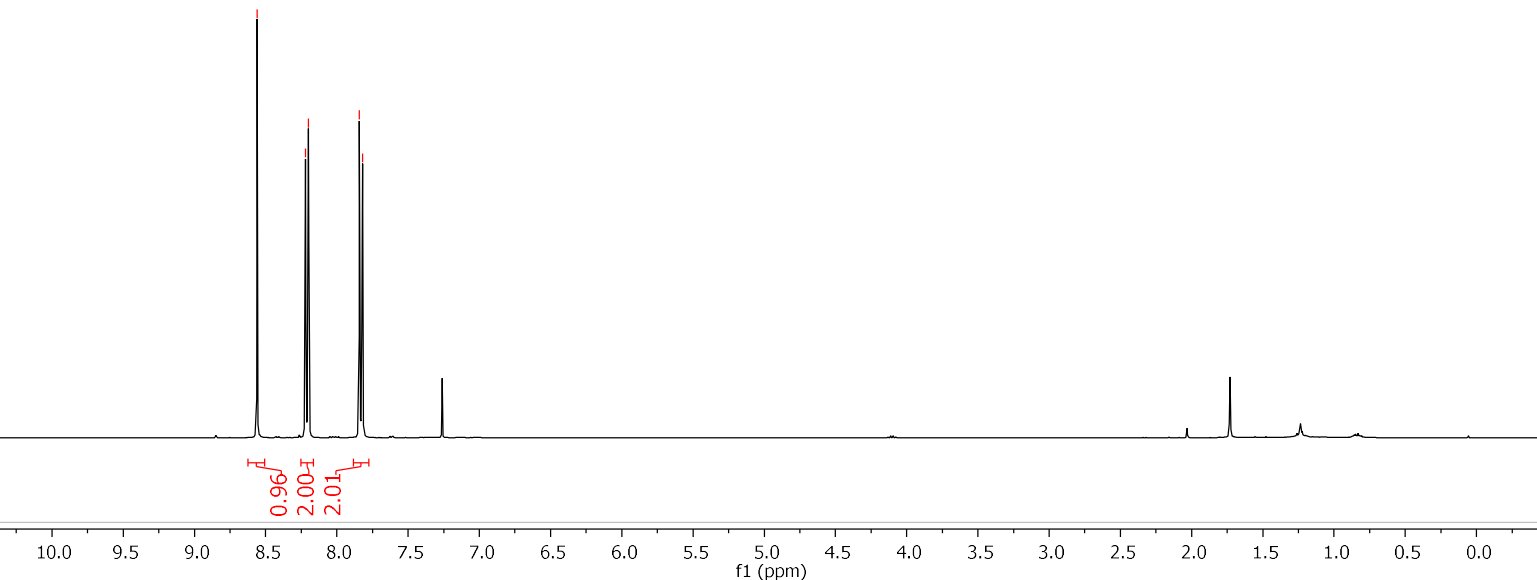

$\mathrm{CDCl} 3,101 \mathrm{MHz}$

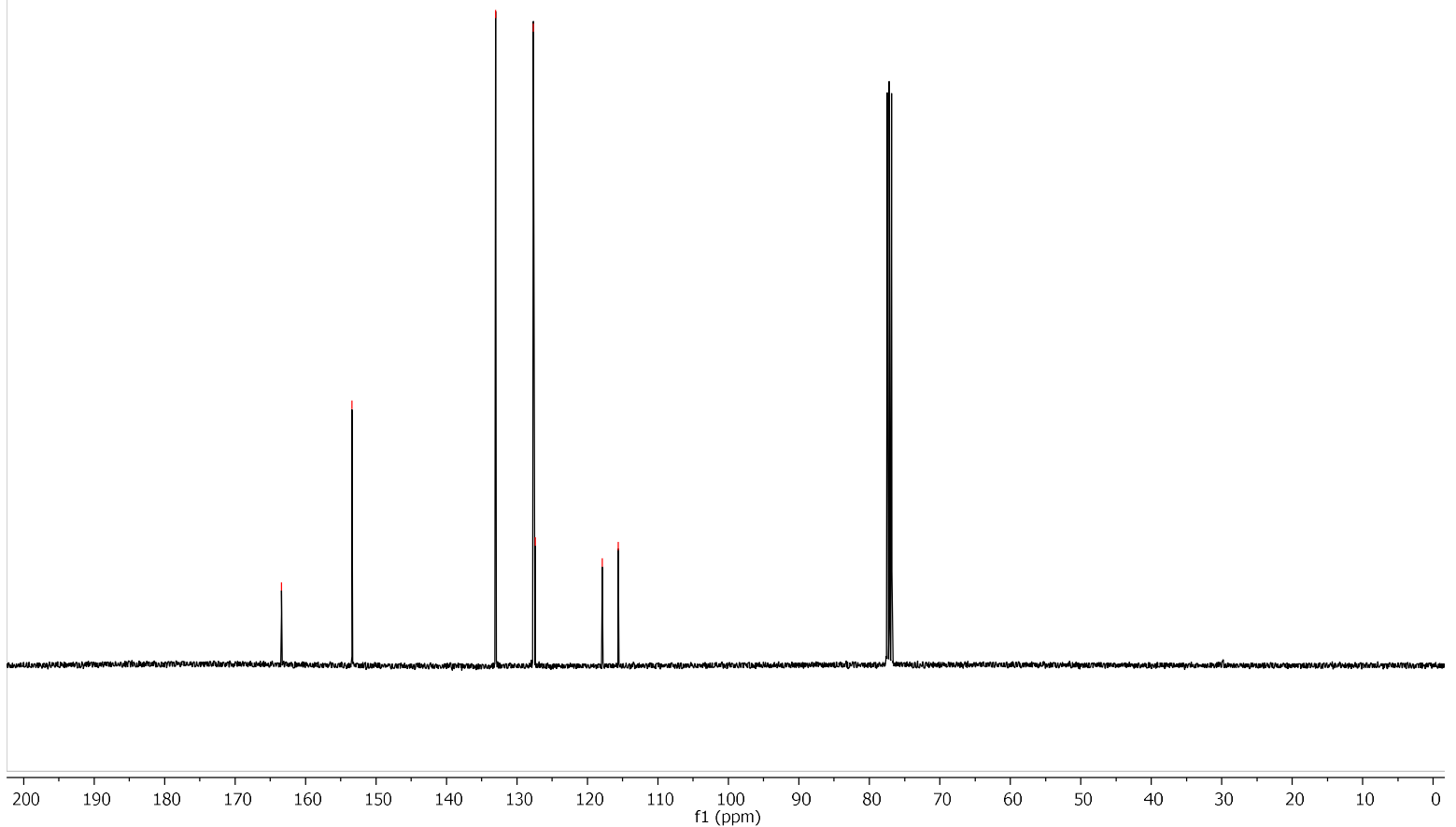


2-(4-(Trifluoromethyl)phenyl)-1,3,4-oxadiazole (14e)
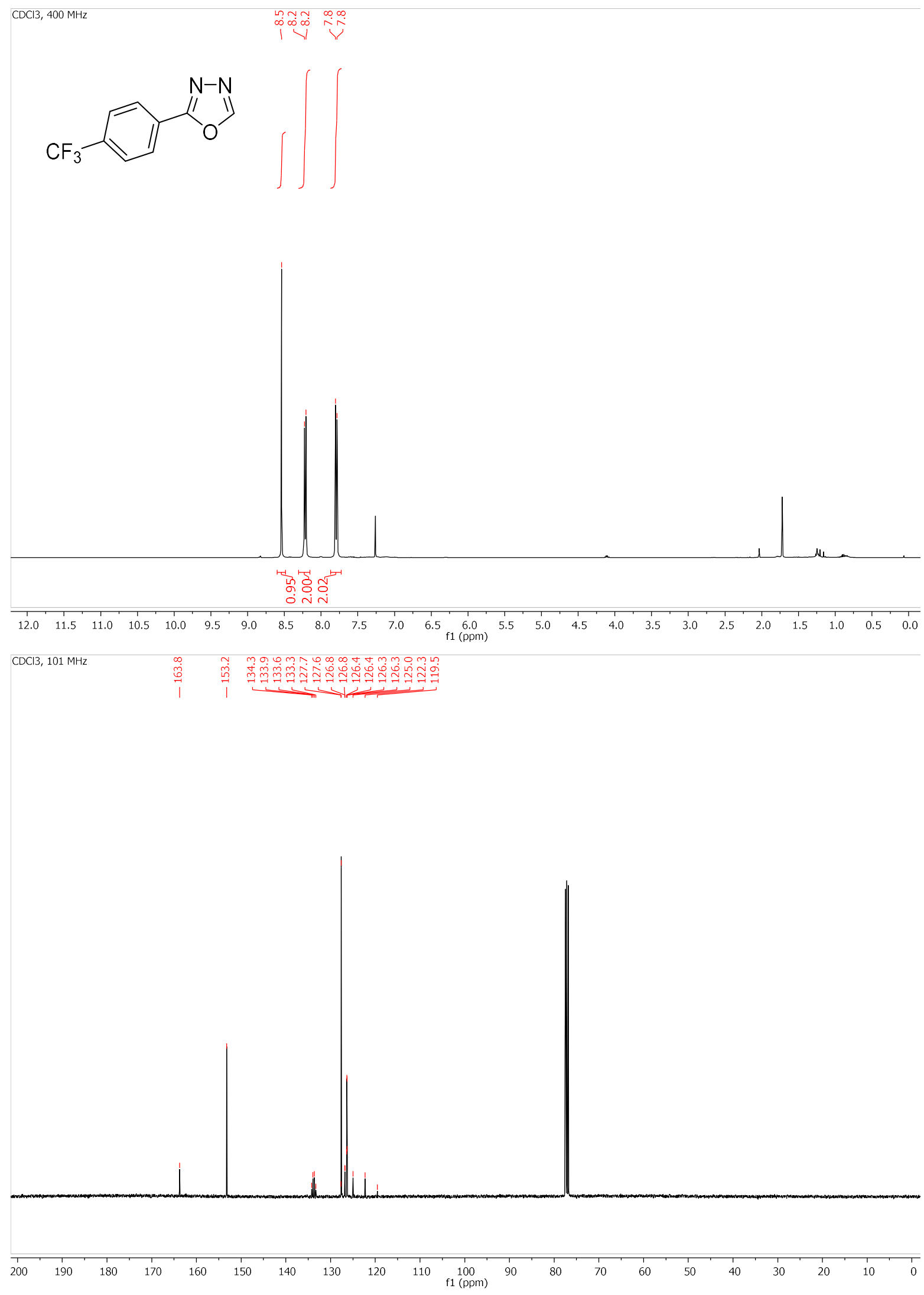

SI 42 
2-(4-Methoxyphenyl)-1,3,4-oxadiazole (14f)
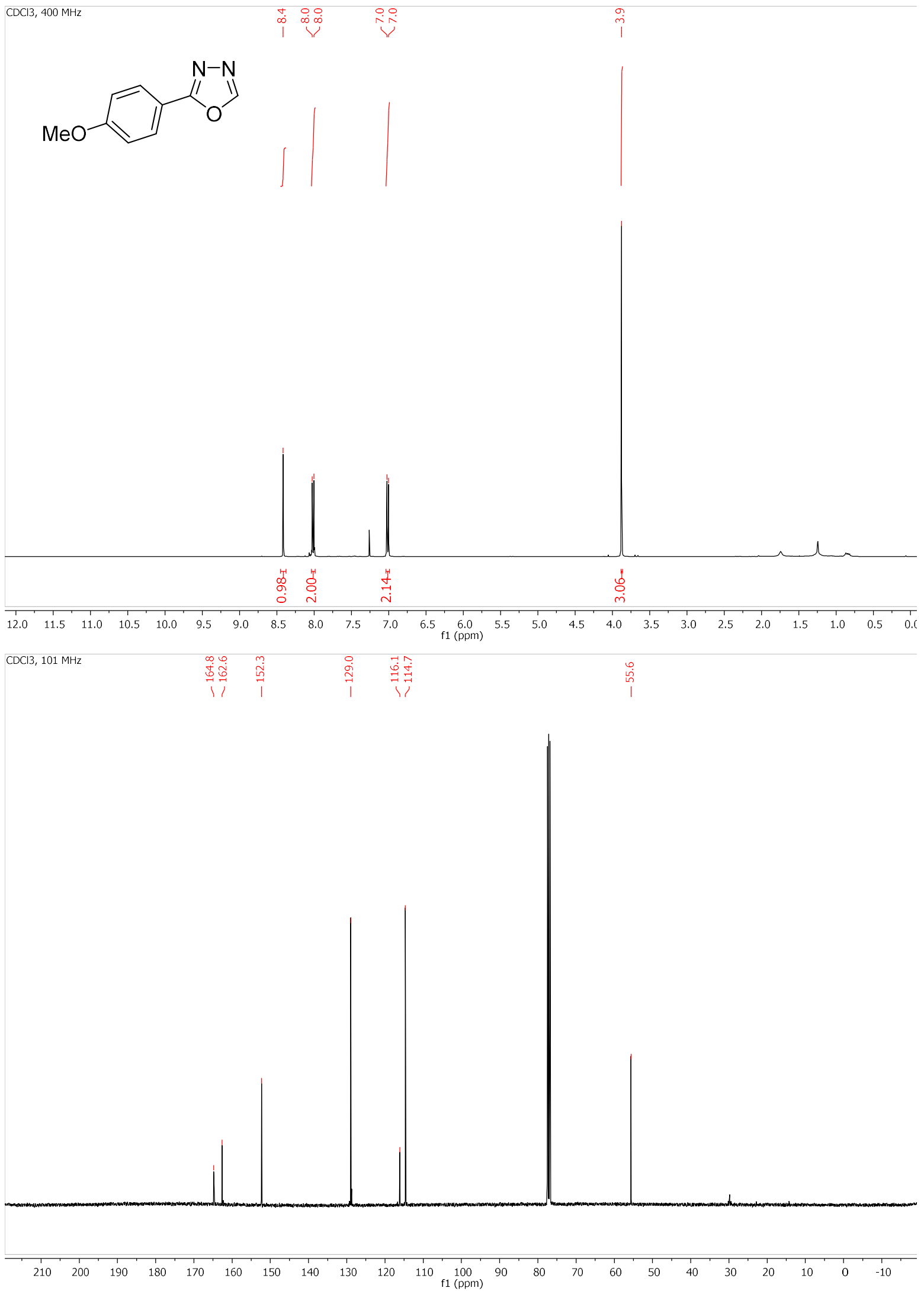
$N, N$-Dimethyl-4-(1,3,4-oxadiazol-2-yl)aniline (14g)
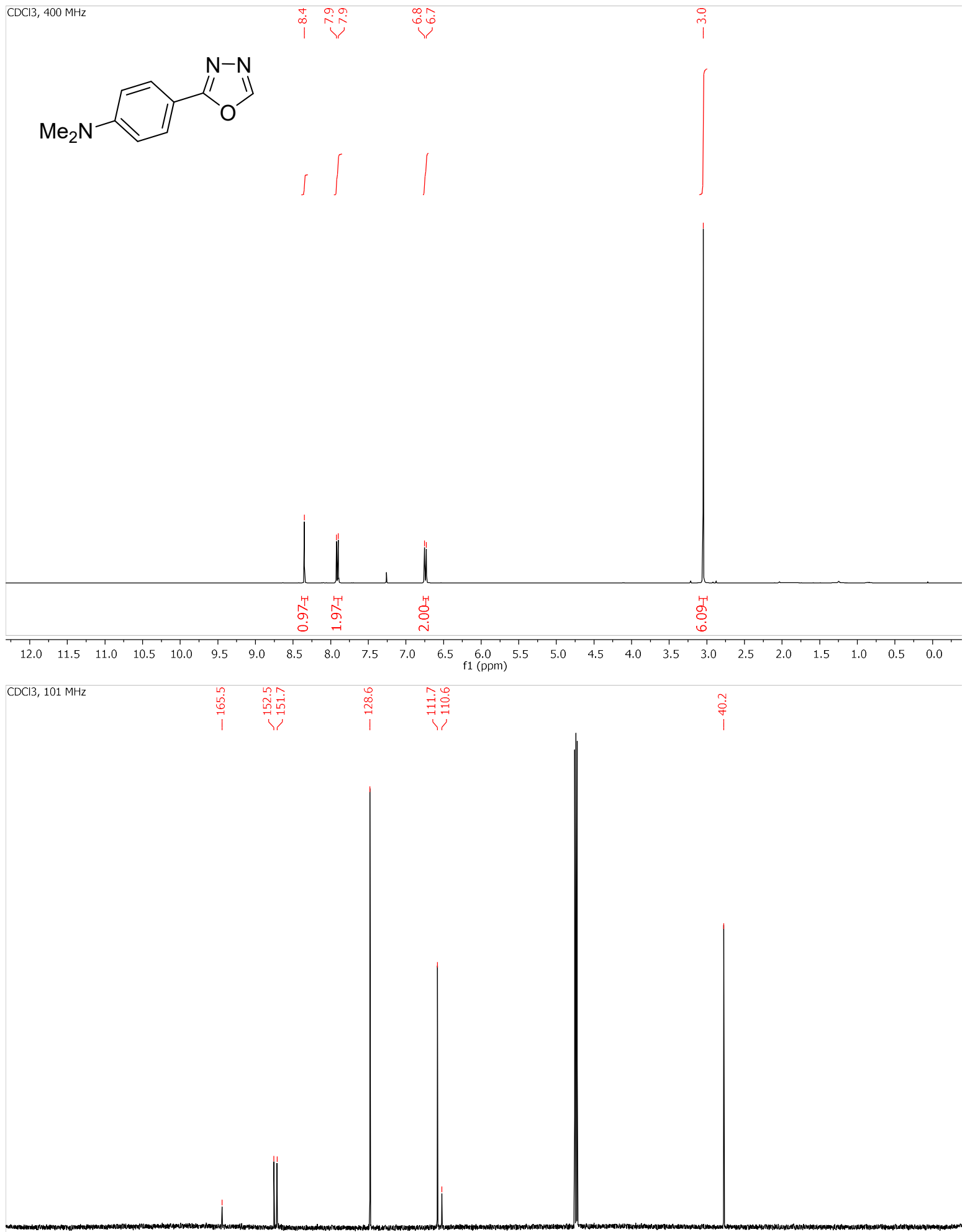

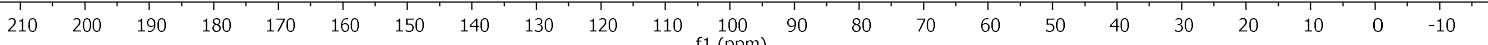


2-(3-Nitrophenyl)-1,3,4-oxadiazole (14h)

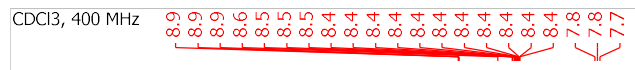
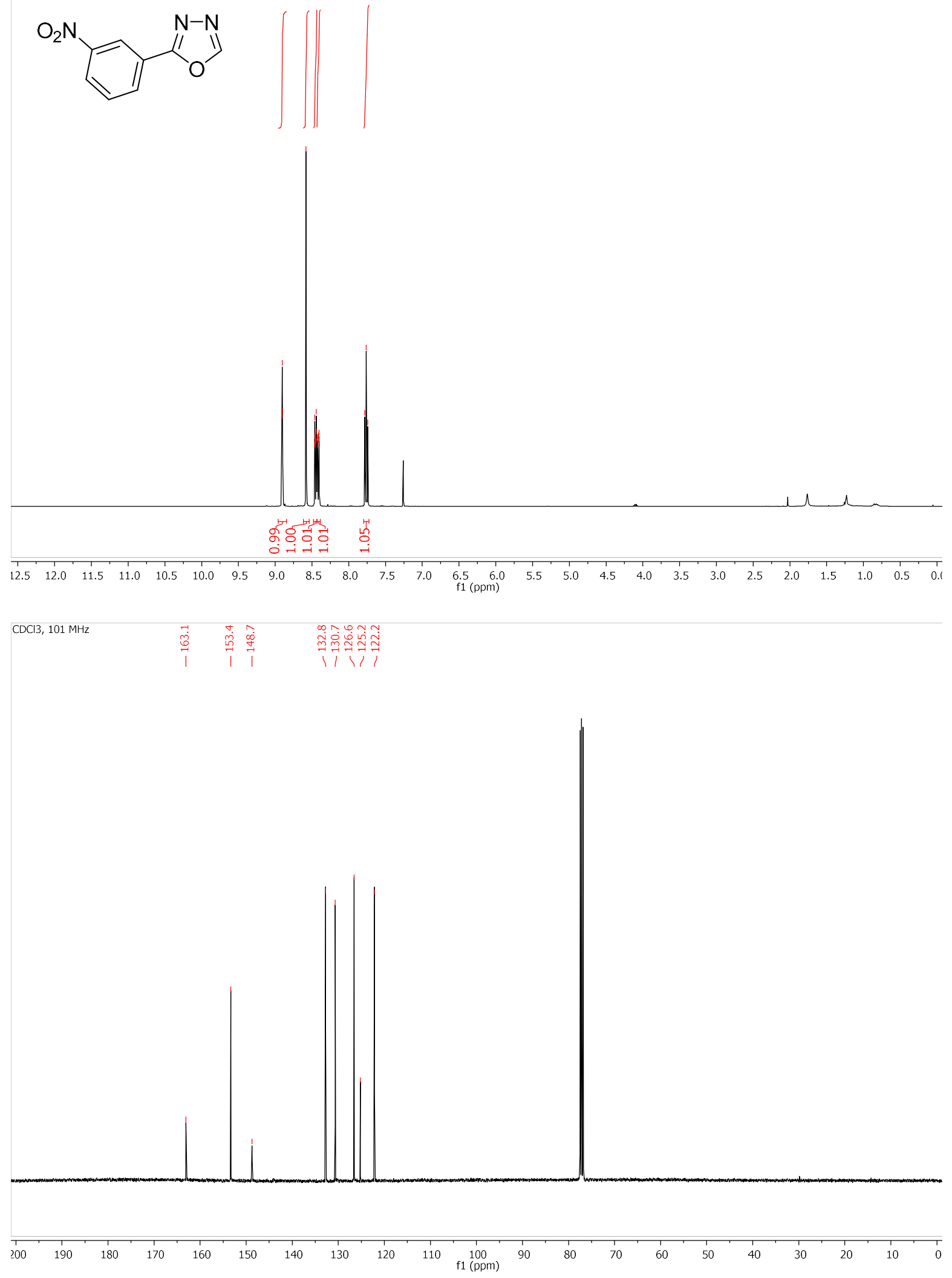

SI 45 
2-(2-Fluorophenyl)-1,3,4-oxadiazole (14i)

$\mathrm{CDCl} 3,400 \mathrm{MHz}$

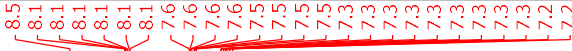

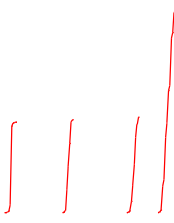<smiles>Fc1ccccc1-c1nnco1</smiles>
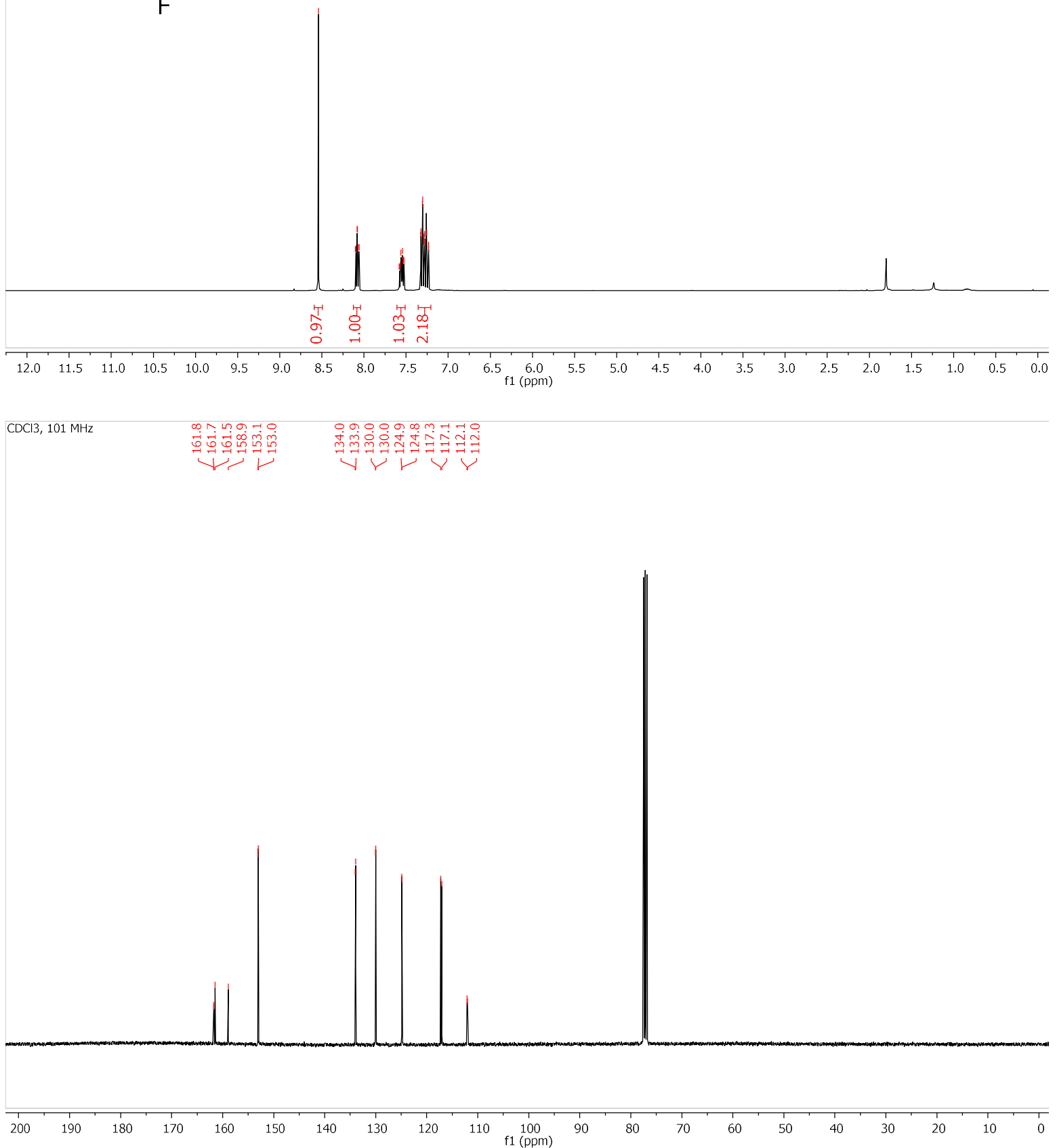
2-(Quinolin-6-yl)-1,3,4-oxadiazole (14j)
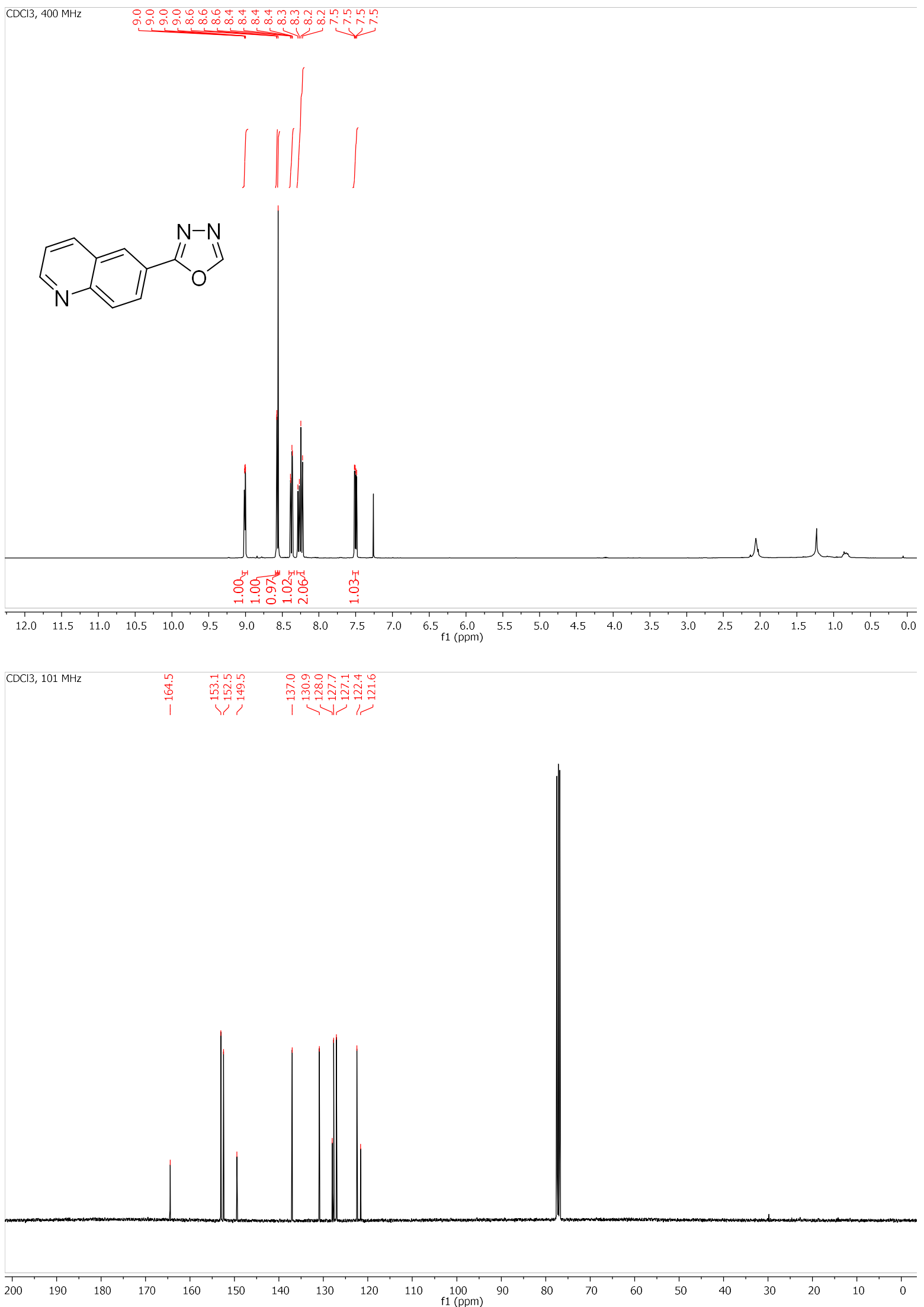
2-(Thiophen-2-yl)-1,3,4-oxadiazole (14k)
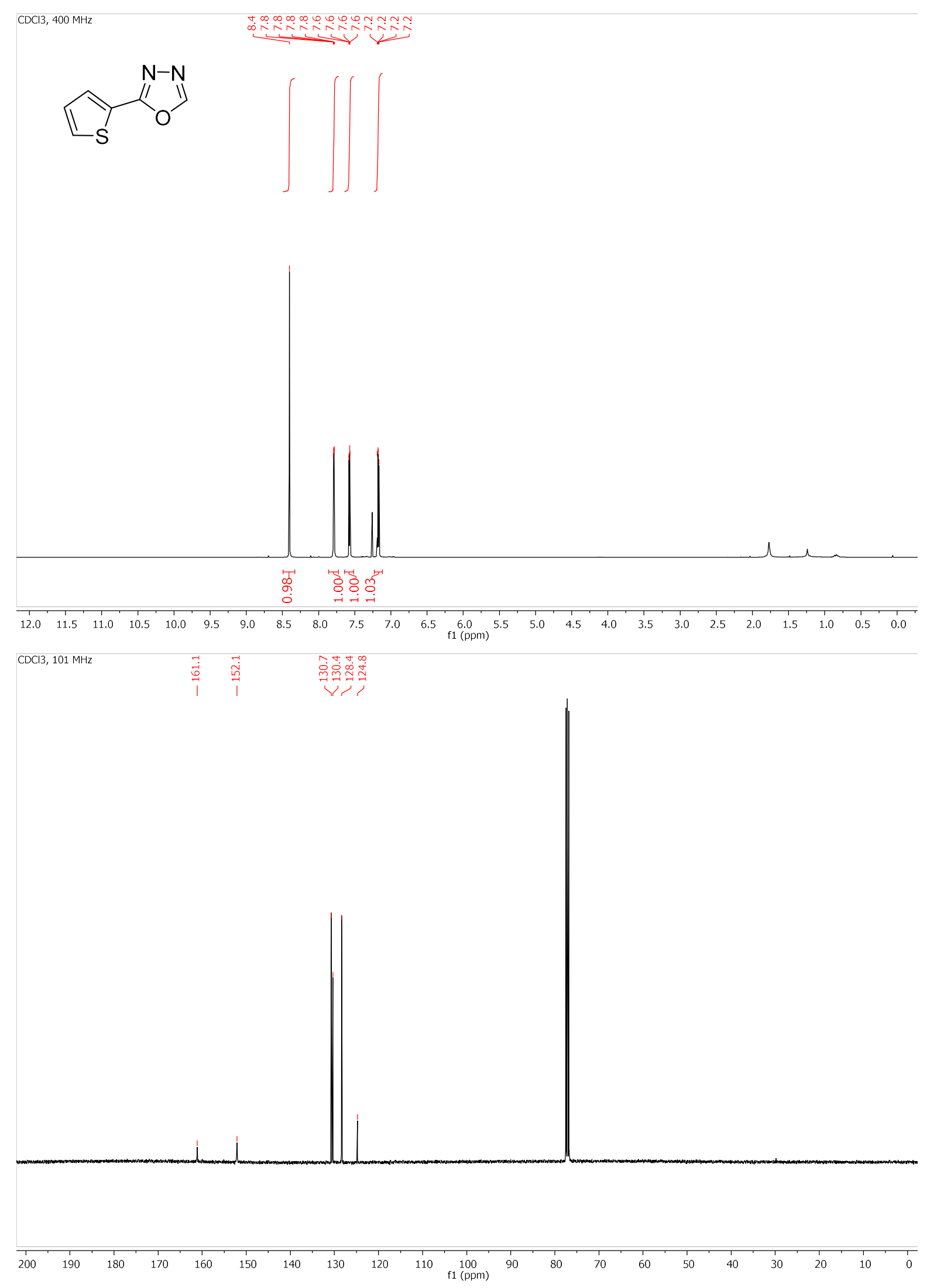

SI 48 
4-(5-Phenyl-1,3,4-oxadiazol-2-yl)morpholine (16a)
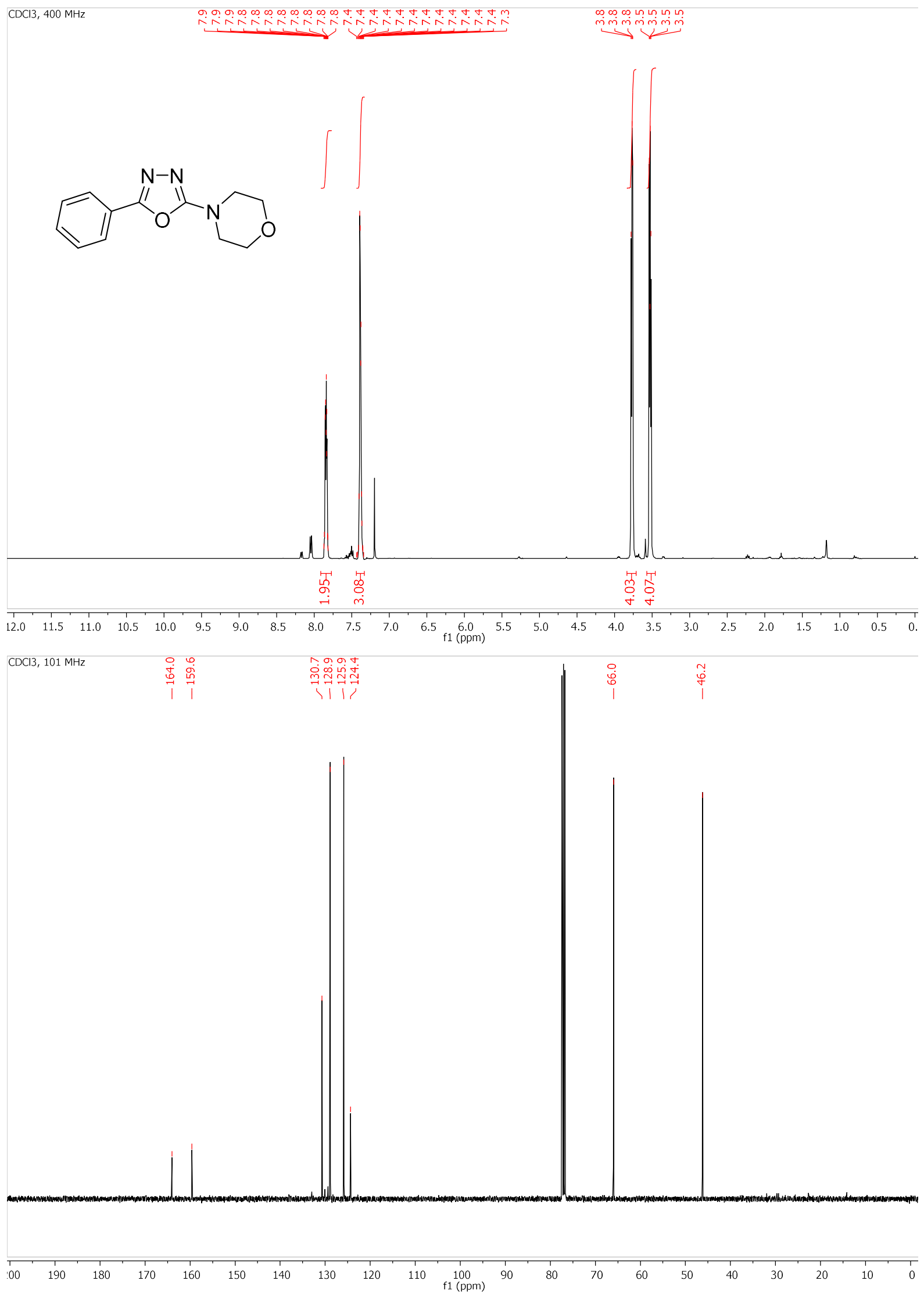
$N, N$-Dimethyl-4-(5-morpholino-1,3,4-oxadiazol-2-yl)aniline (16b)

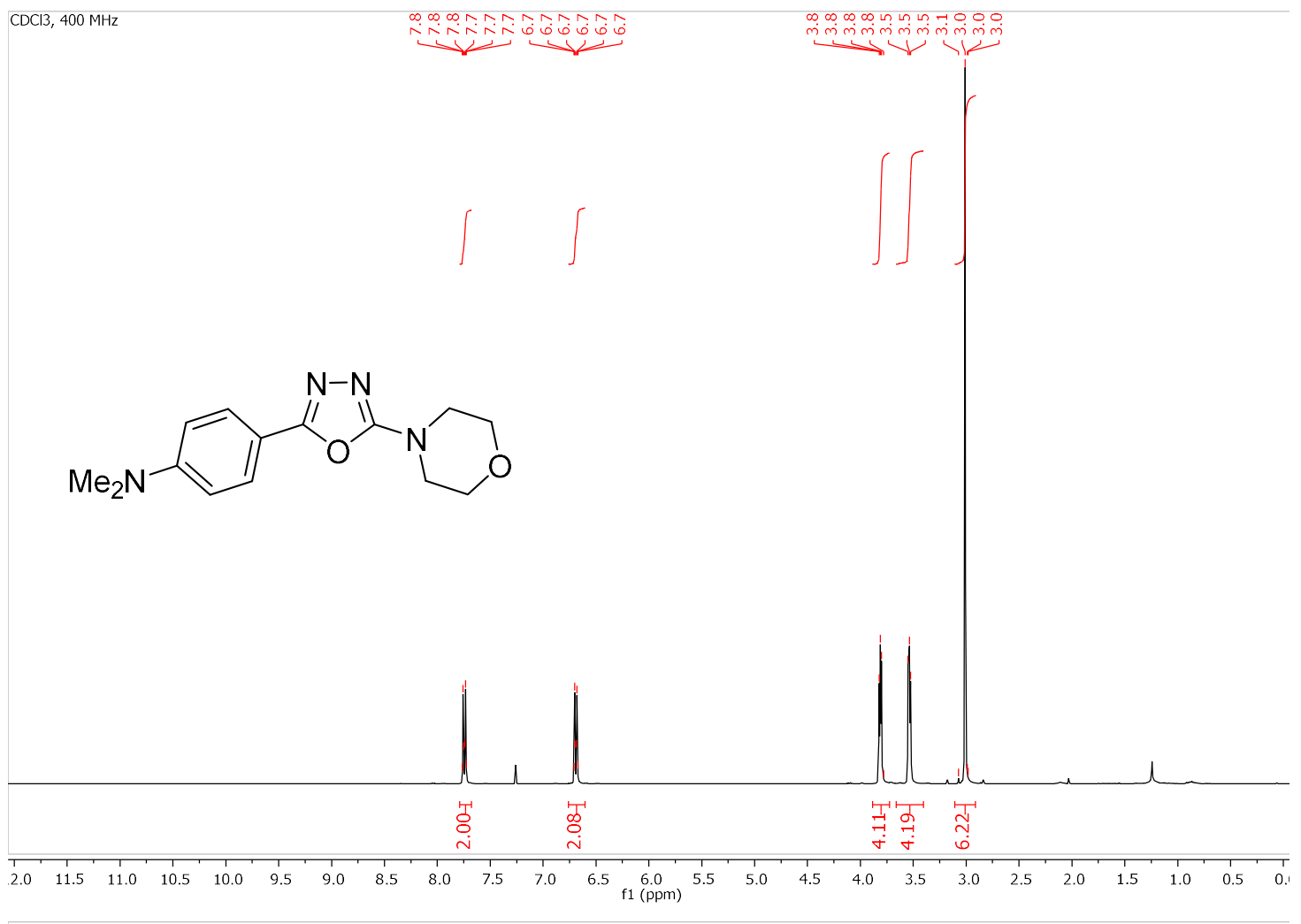

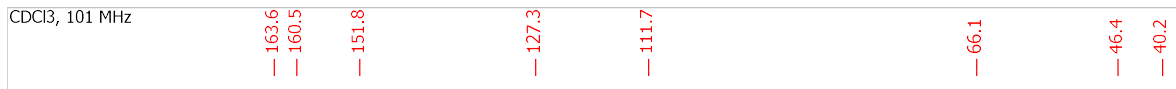

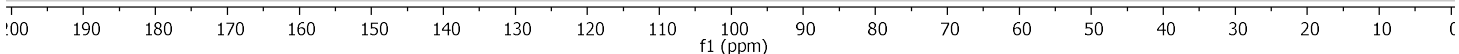


4-(5-(4-Chlorophenyl)-1,3,4-oxadiazol-2-yl)morpholine (16c)
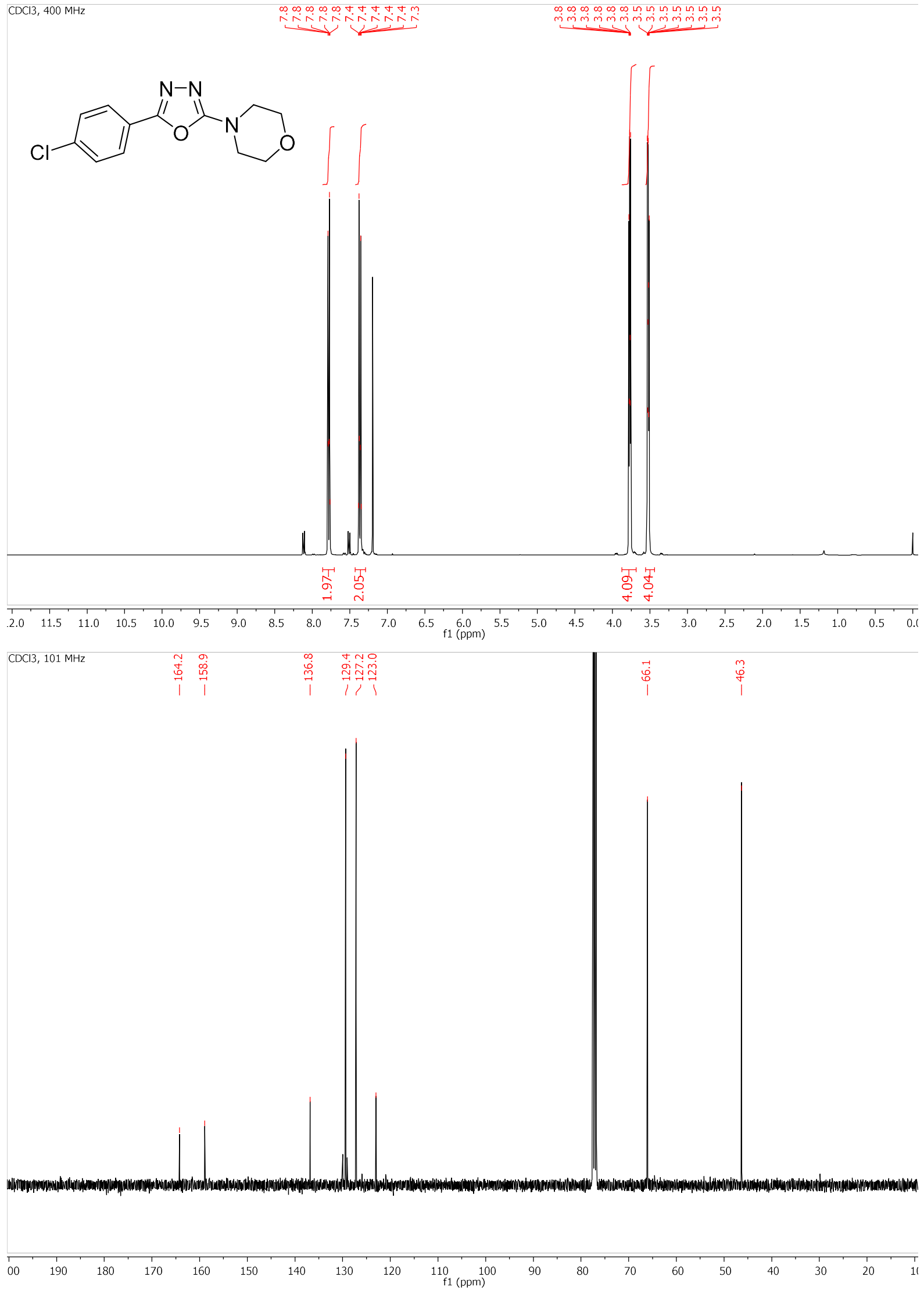
4-(5-(4-(Trifluoromethyl)phenyl)-1,3,4-oxadiazol-2-yl)morpholine (16d)

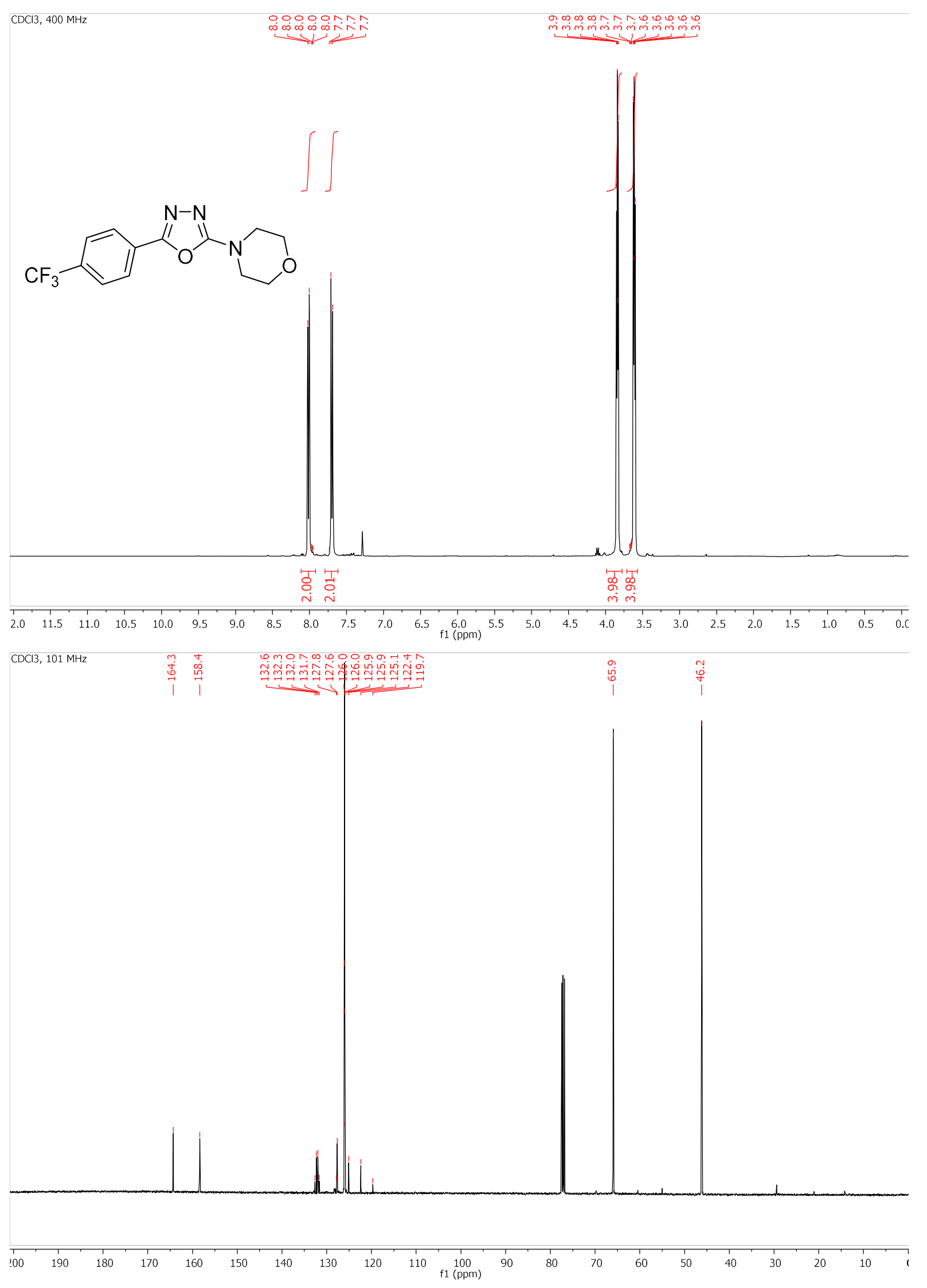

SI 52 
$N, N$-Diallyl-5-phenyl-1,3,4-oxadiazol-2-amine (16e)

$\mathrm{CDCl} 3,400 \mathrm{MHz}$

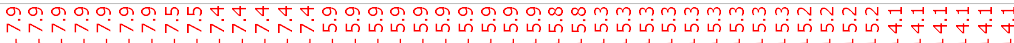
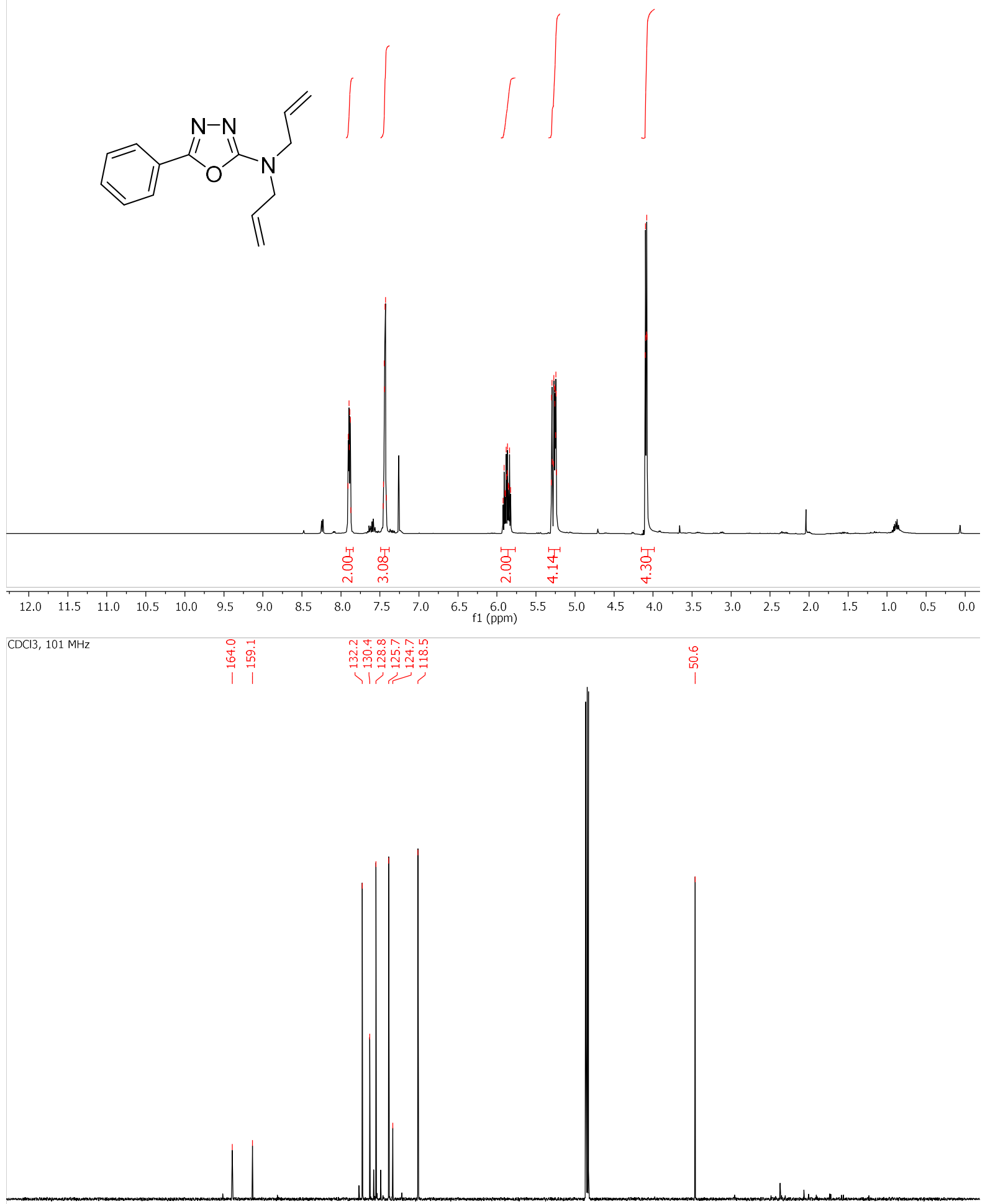

$\begin{array}{lllllllllllllllllllllll}210 & 200 & 190 & 180 & 170 & 160 & 150 & 140 & 130 & 120 & 110 & \underset{\mathrm{f} 1}{100}(\mathrm{ppm}) & 90 & 80 & 70 & 60 & 50 & 40 & 30 & 20 & 10 & 0 & -10\end{array}$ 
2-(Azepan-1-yl)-5-(4-methoxyphenyl)-1,3,4-oxadiazole (16f)
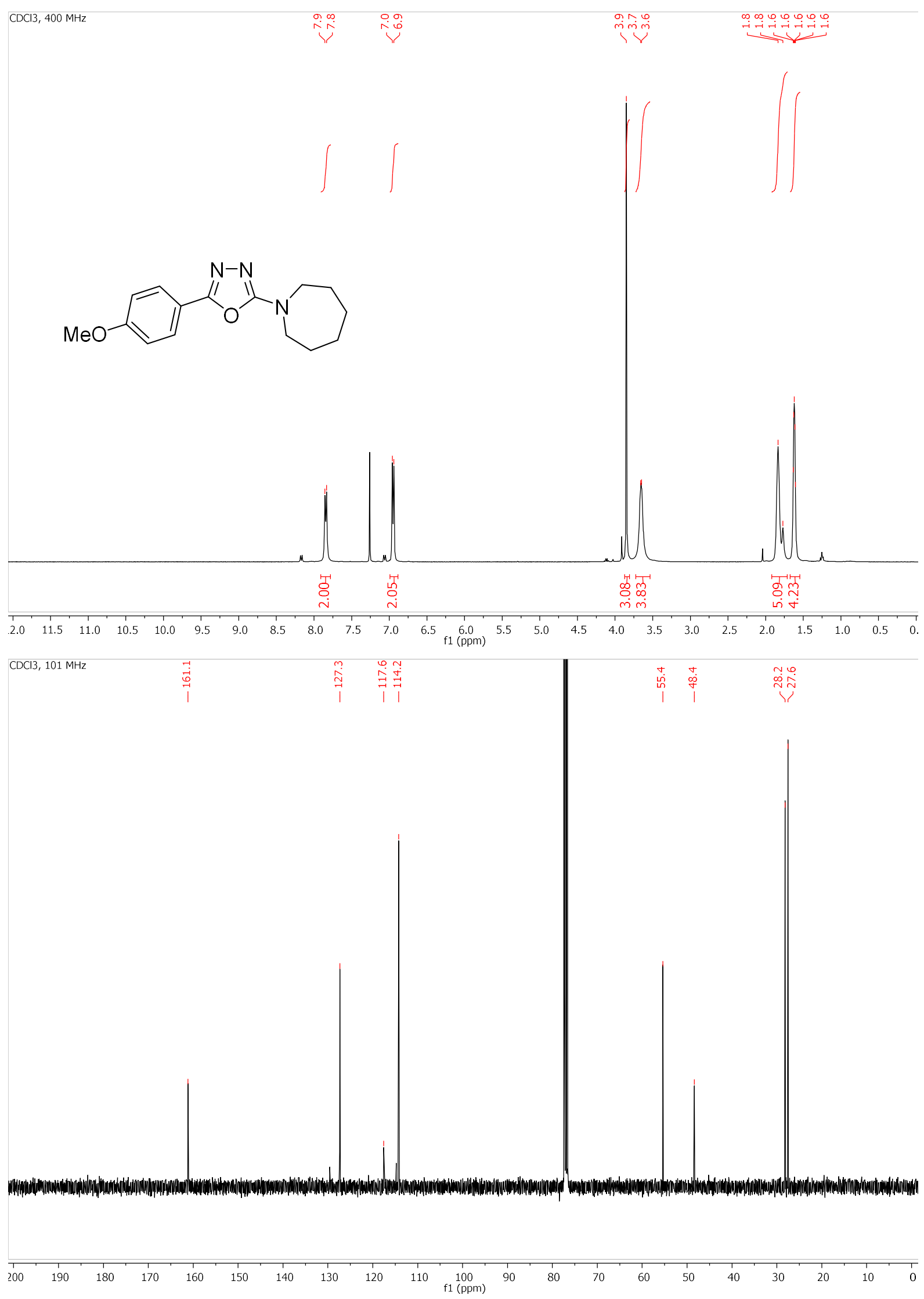

SI 54 
2-Phenyl-5-(4-(pyrimidin-2-yl)piperazin-1-yl)-1,3,4-oxadiazole (16g)
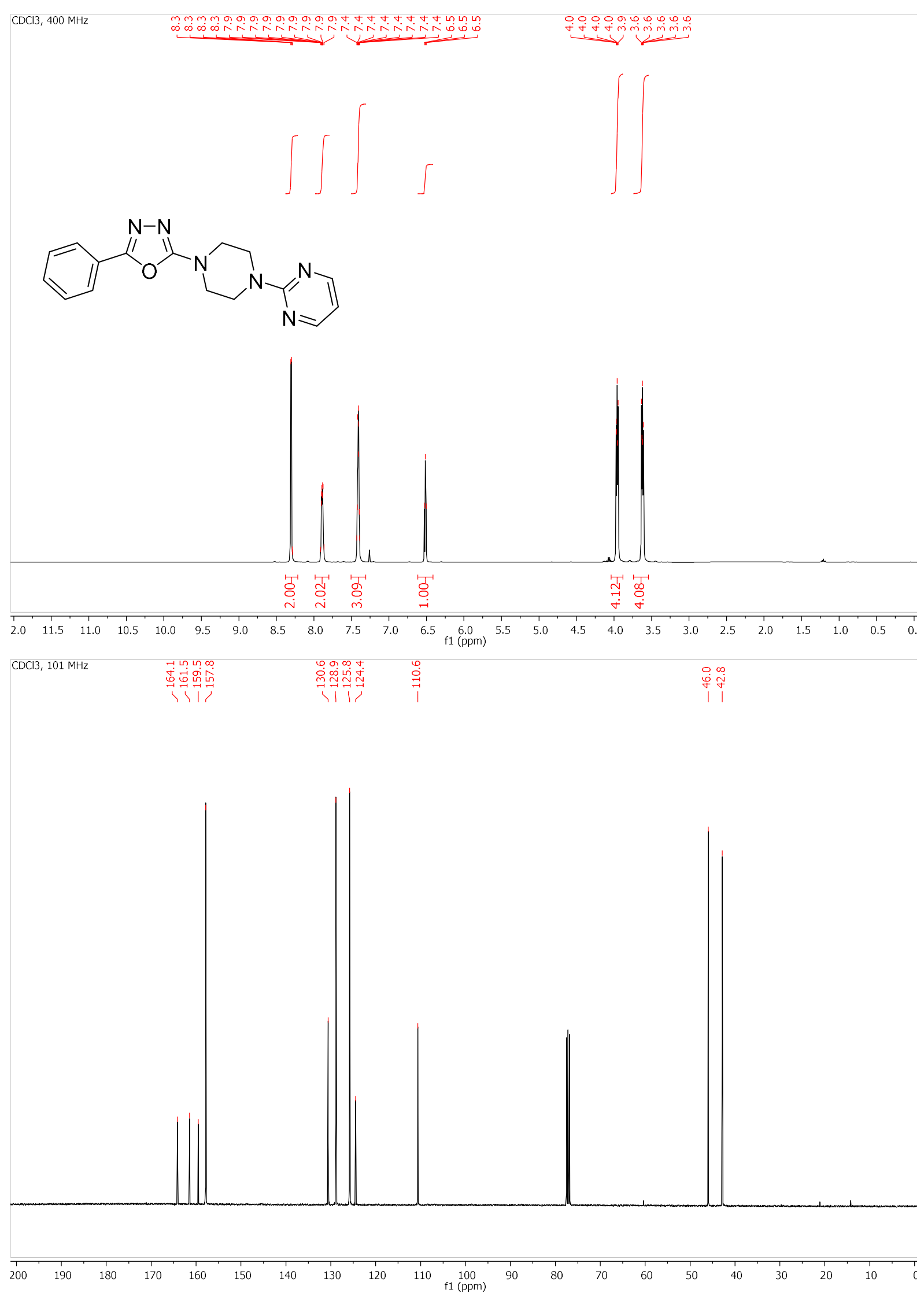

SI 55 
<smiles>CCOC(=O)C1CCCN(c2nnc(-c3ccc(Cl)cc3)o2)C1</smiles>
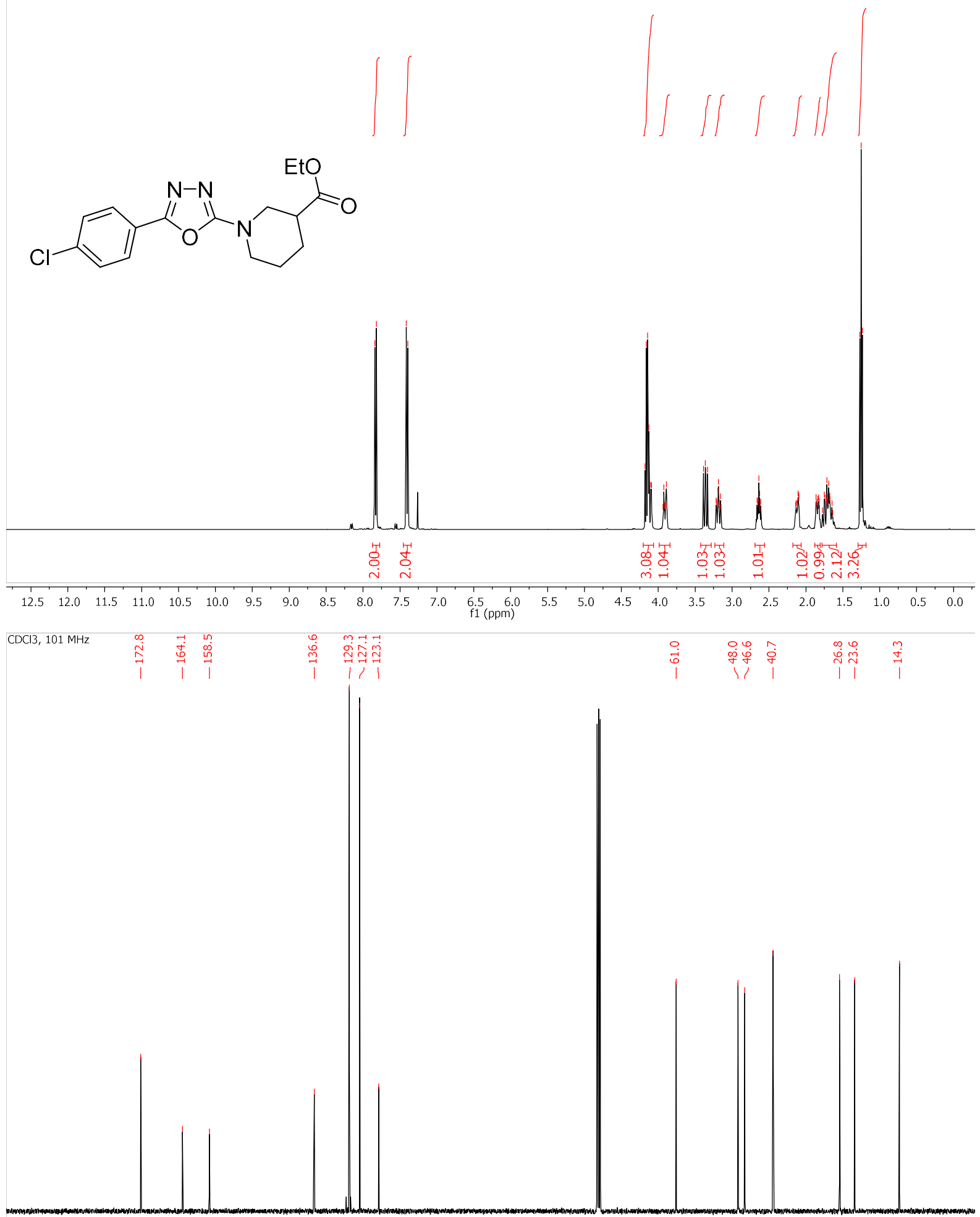
8-(5-(4-Methoxyphenyl)-1,3,4-oxadiazol-2-yl)-1,4-dioxa-8-azaspiro[4.5]decane (16i)

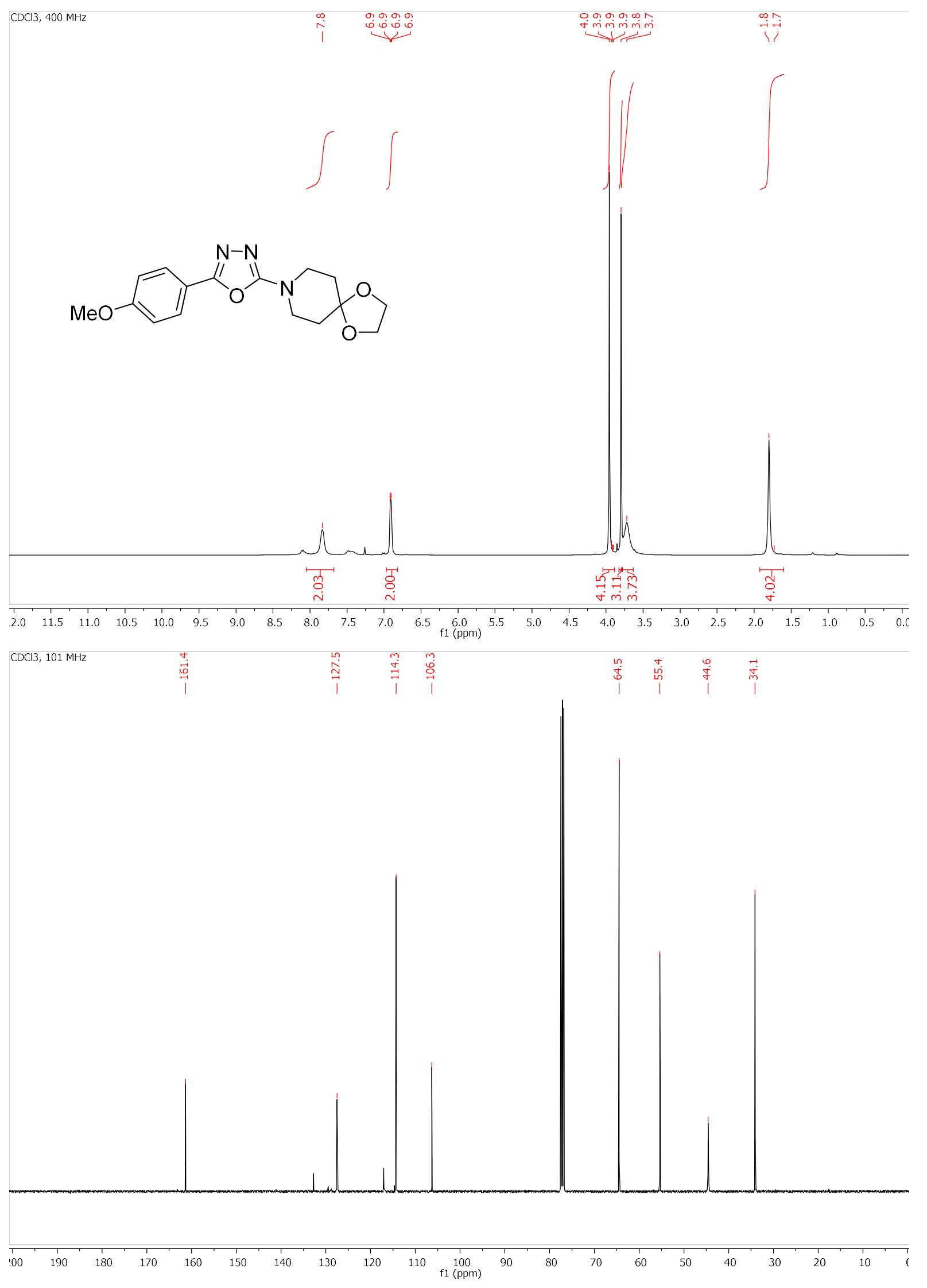

SI 57 
4-(5-(1,4-Dioxa-8-azaspiro[4.5]decan-8-yl)-1,3,4-oxadiazol-2-yl)-N,N-dimethylaniline $(16 j)$
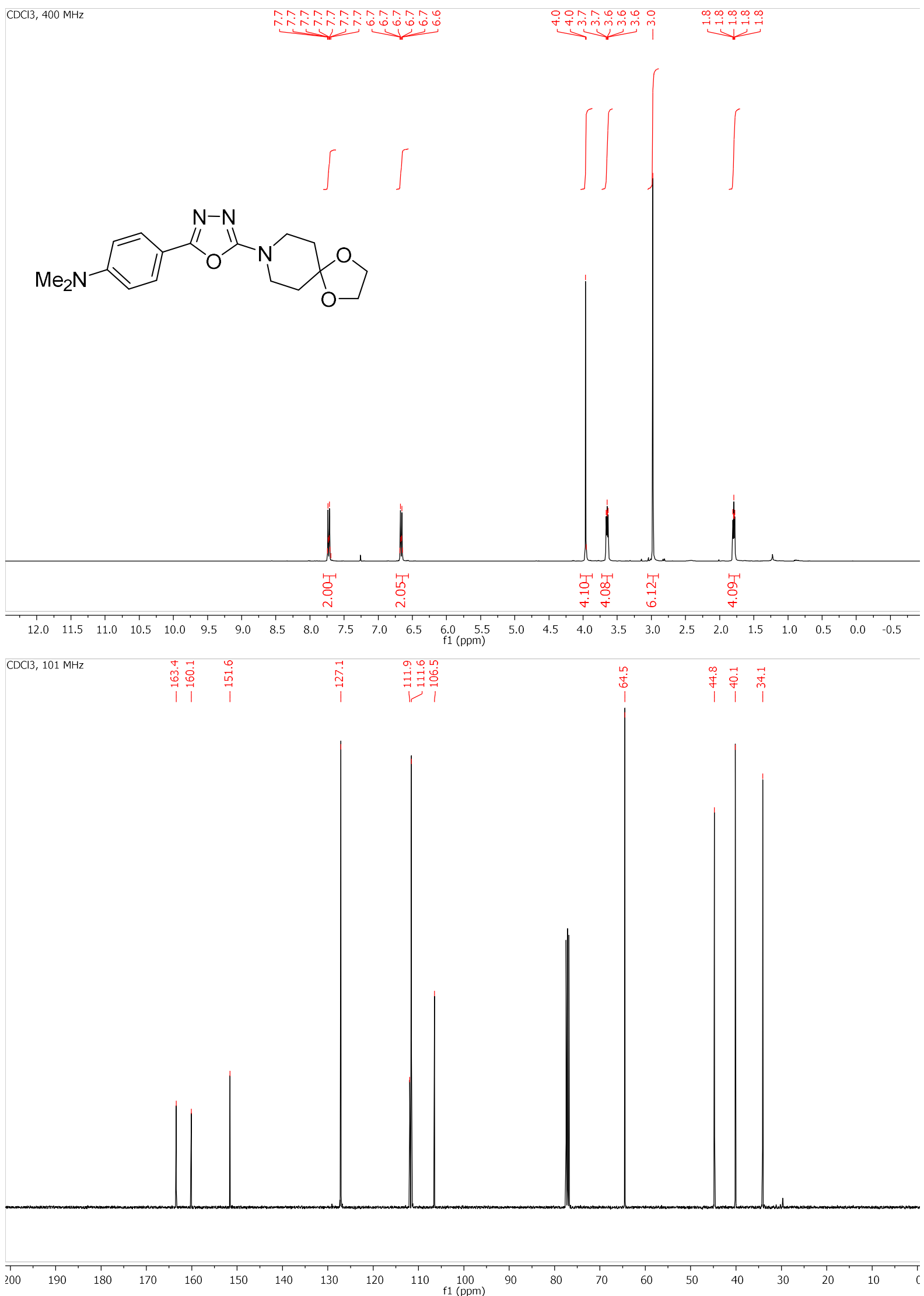
N-(1-(5-Phenyl-1,3,4-oxadiazol-2-yl)piperidin-3-yl)cyclopropanecarboxamide (16k)

CDCl, 400 MHz
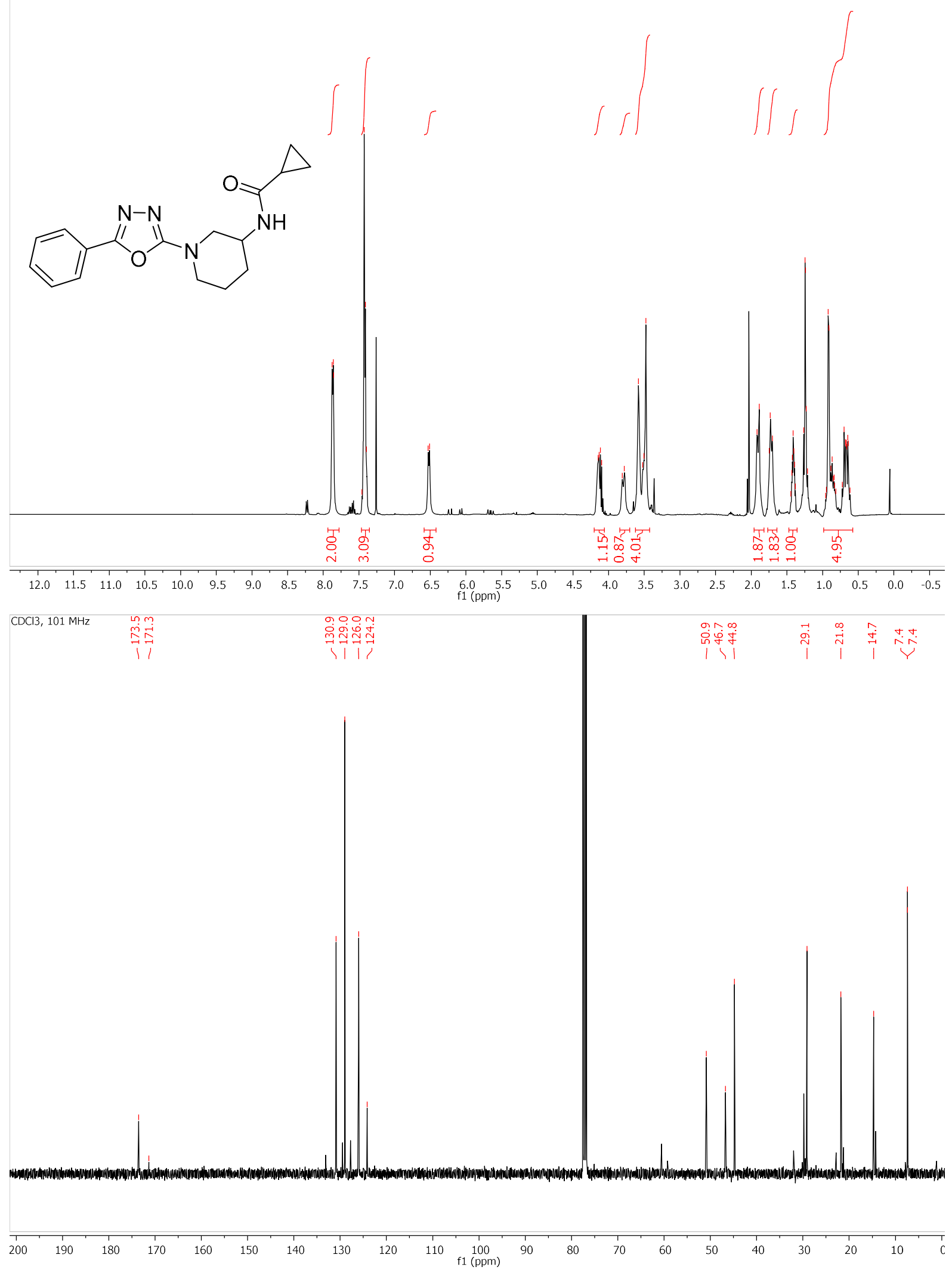

SI 59 
5-lodo-1-propyl-1 H-1,2,4-triazole (18a)

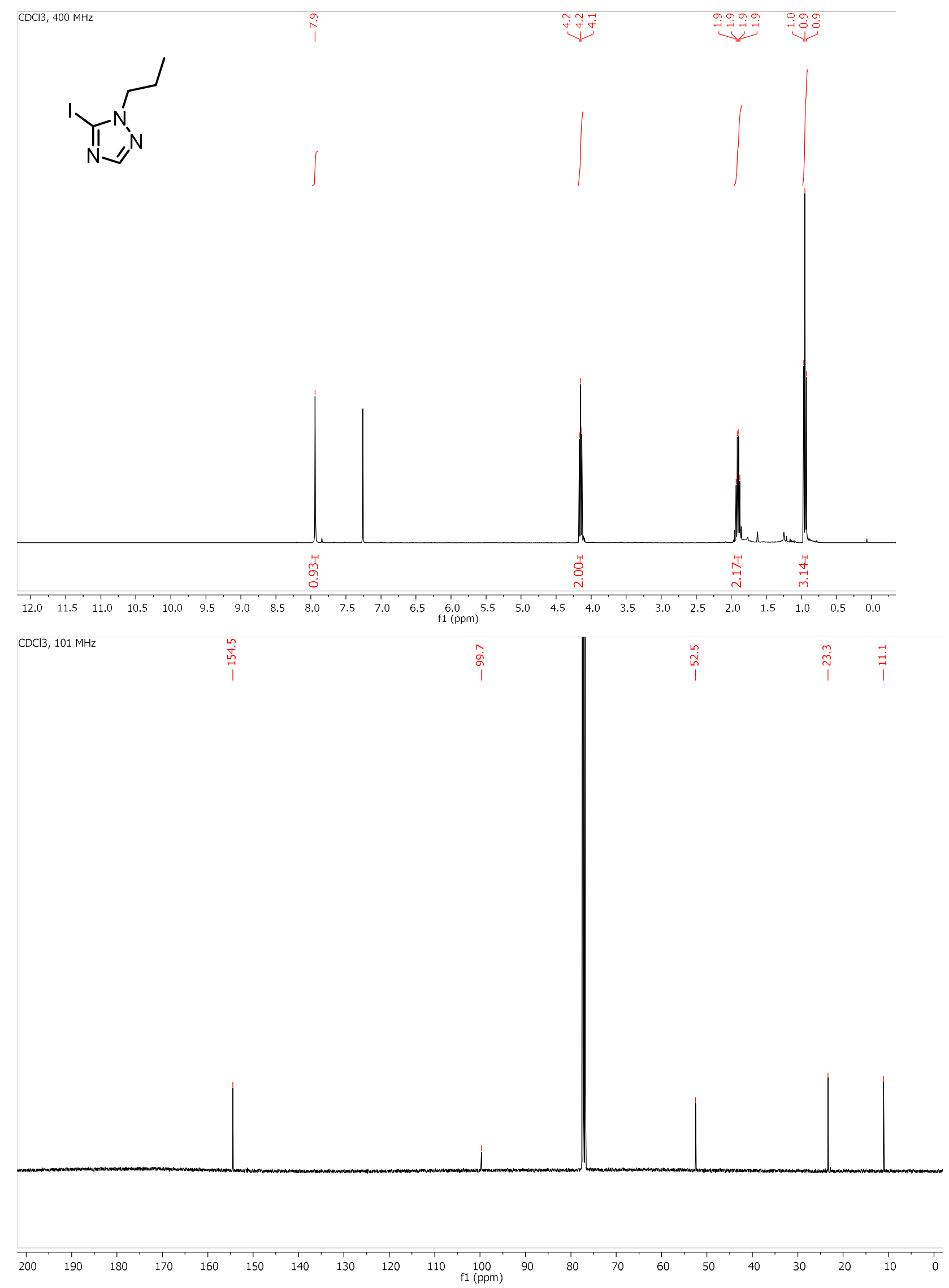

SI 60 
5-Allyl-1-propyl-1H-1,2,4-triazole (18b)

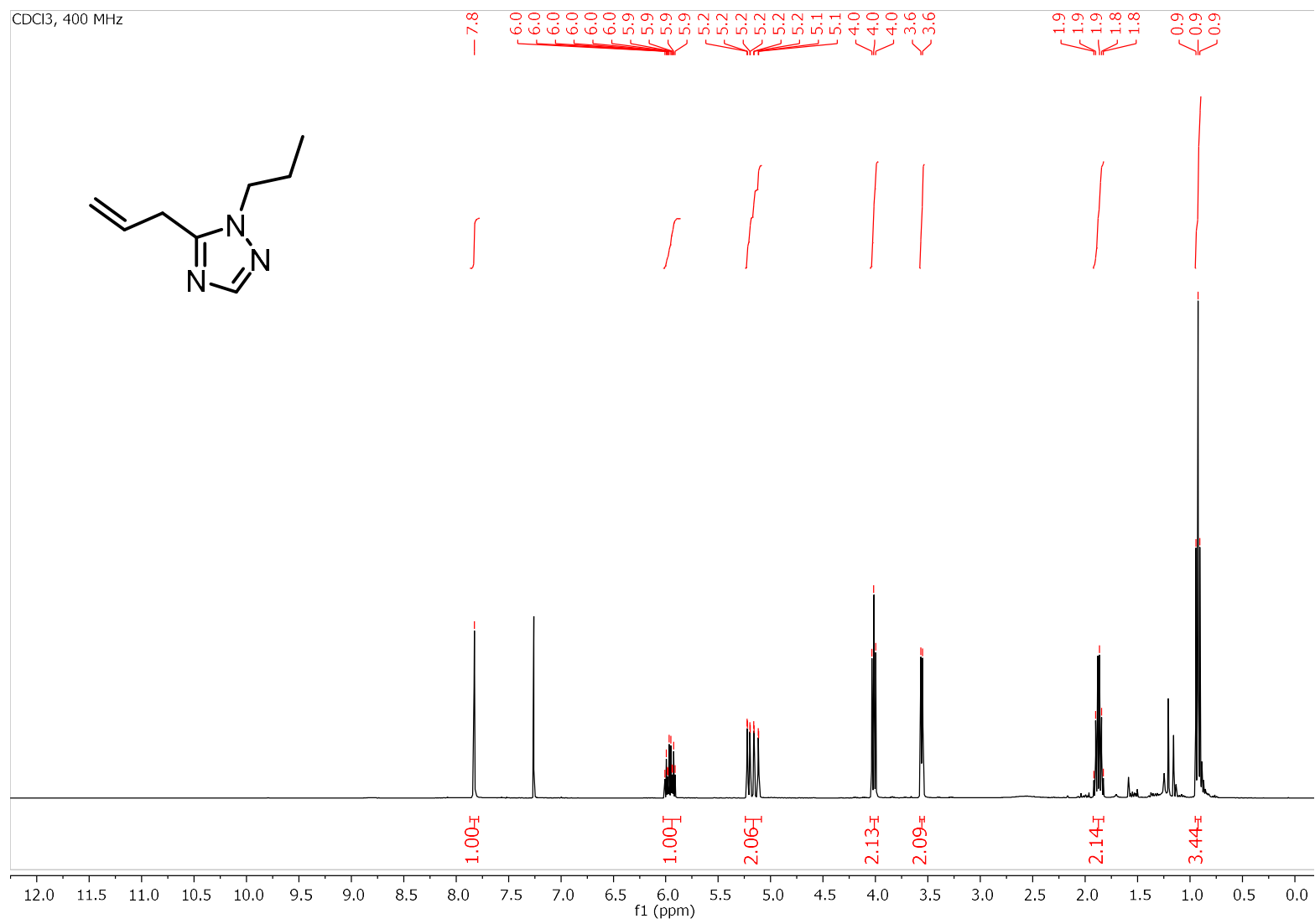

$\mathrm{CDCl}, 101 \mathrm{MHz}$

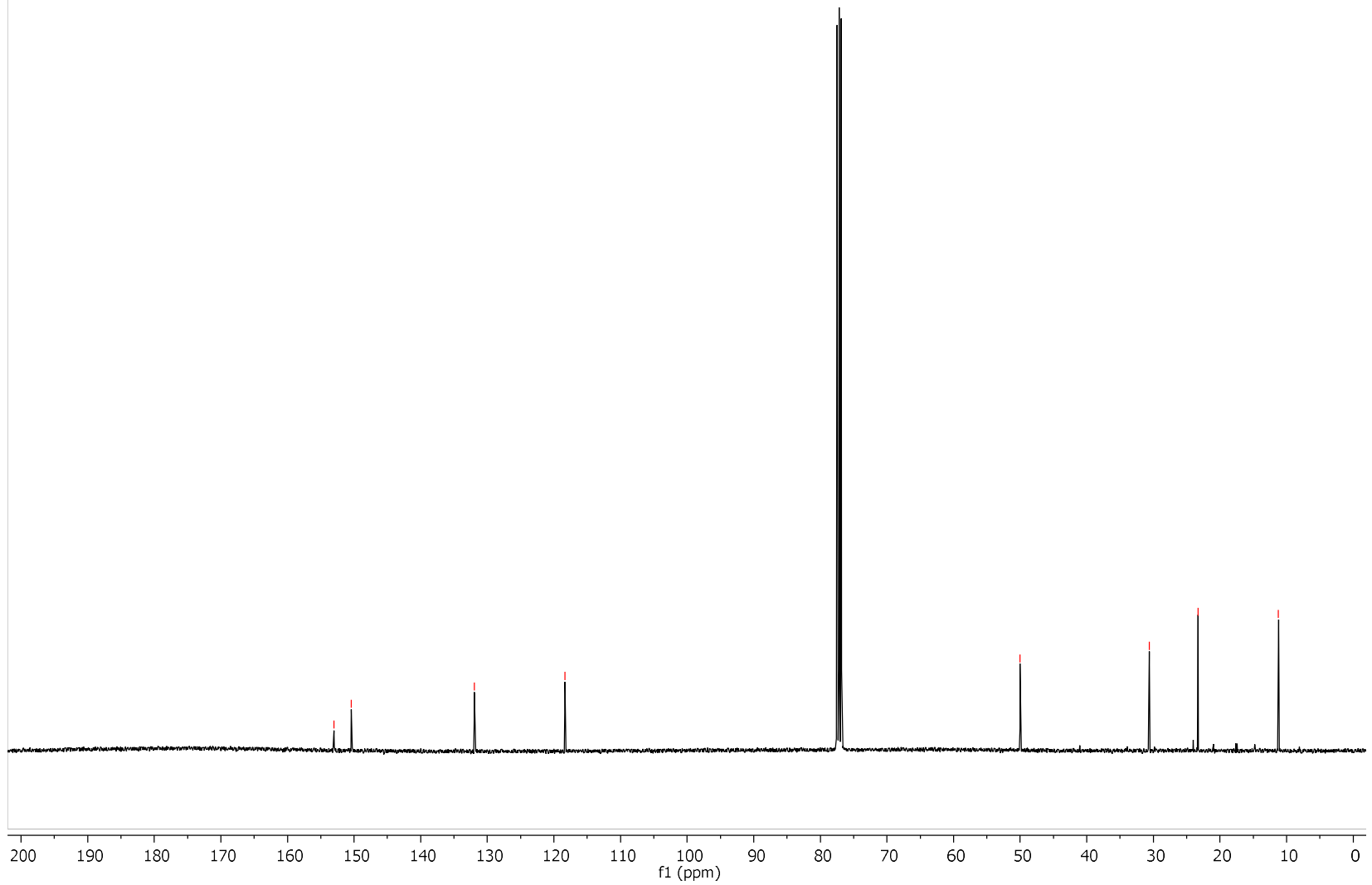


5-(Cyclohex-2-en-1-yl)-1-propyl-1H-1,2,4-triazole (18c)<smiles>CCCn1ncnc1C1C=CCCC1</smiles>
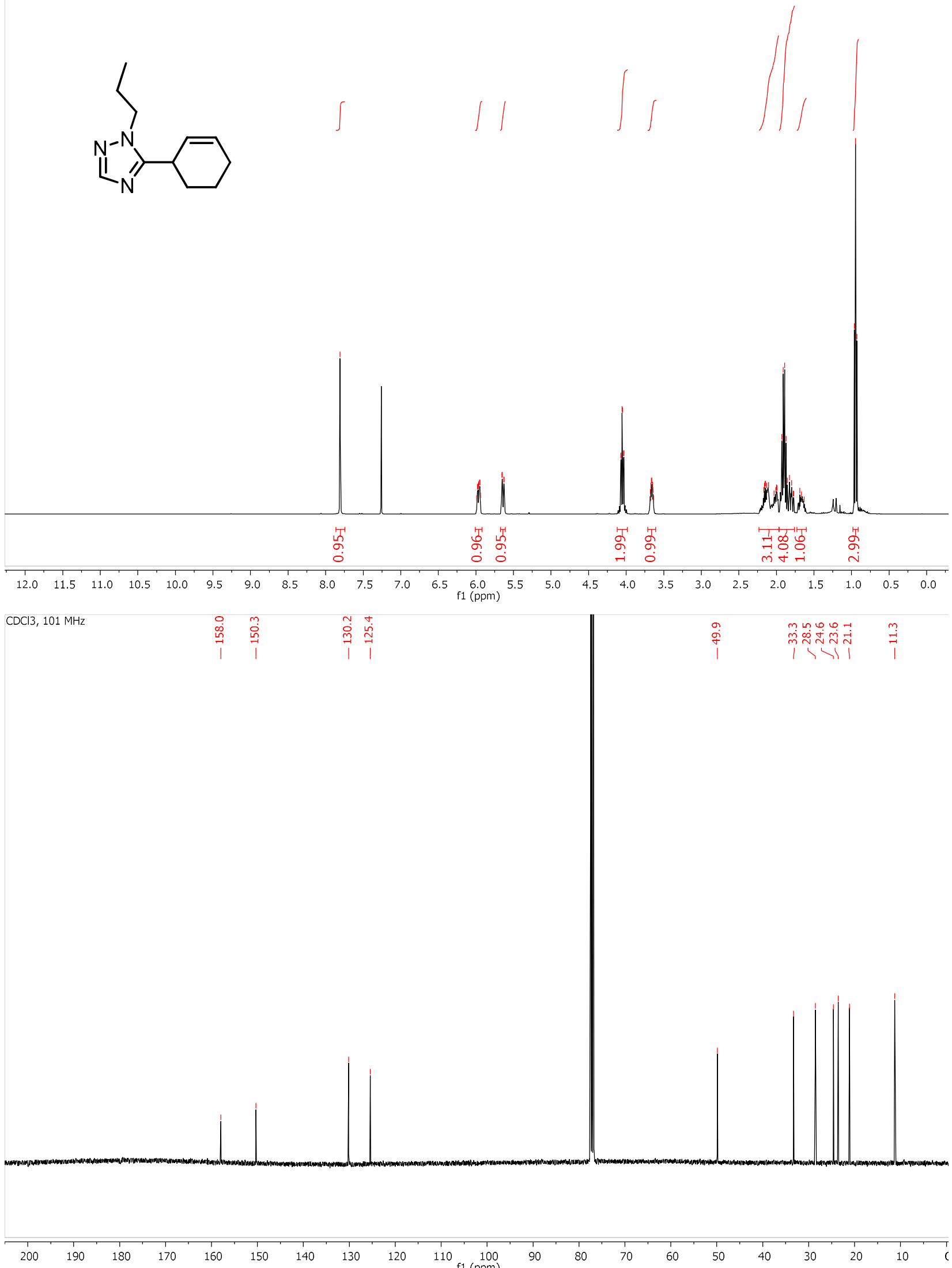
5-(Cyclohex-2-en-1-yl)-1-benzyl-1H-1,2,4-triazole (18d)

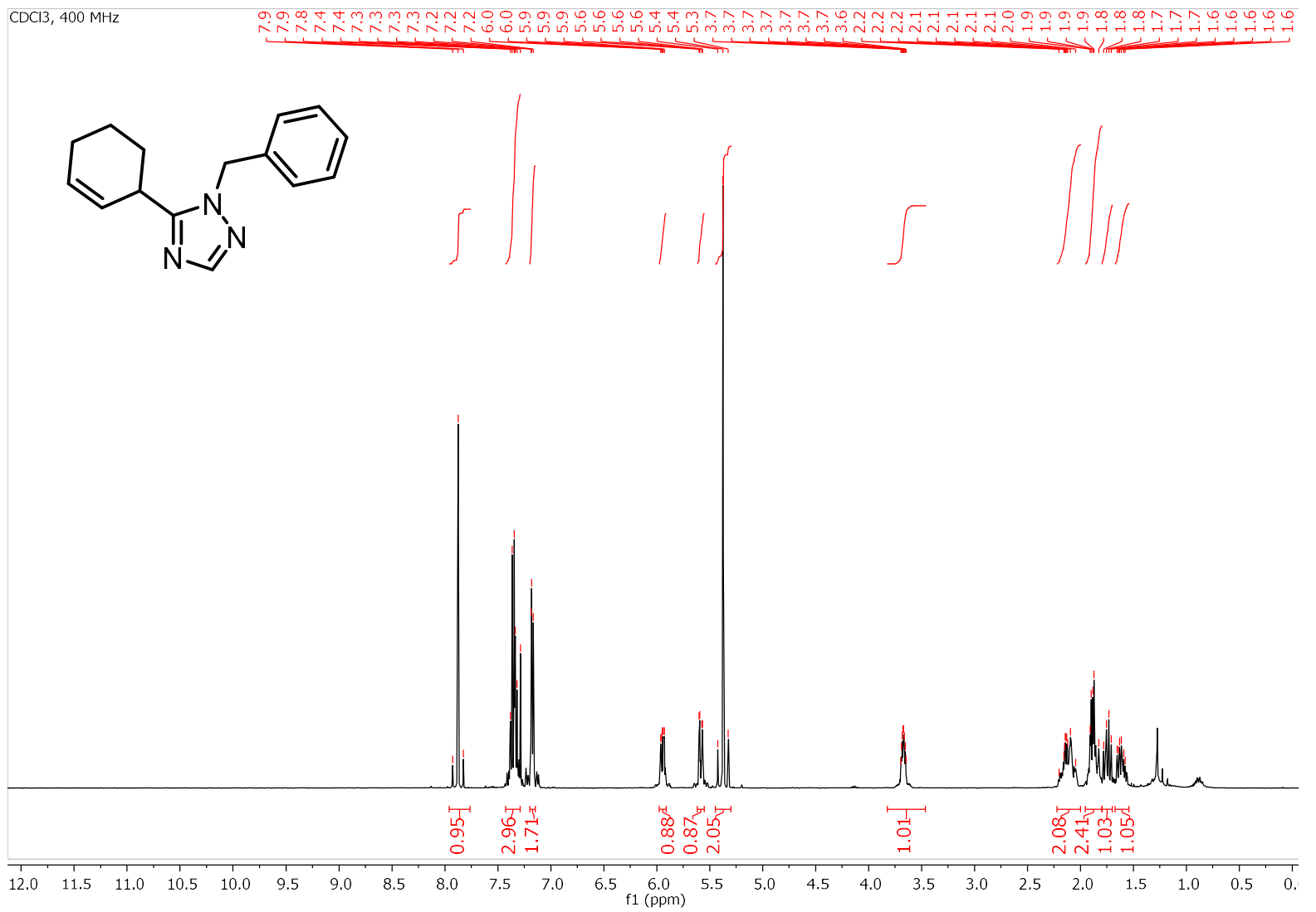

$\mathrm{CDCl}, 101 \mathrm{MHz}$

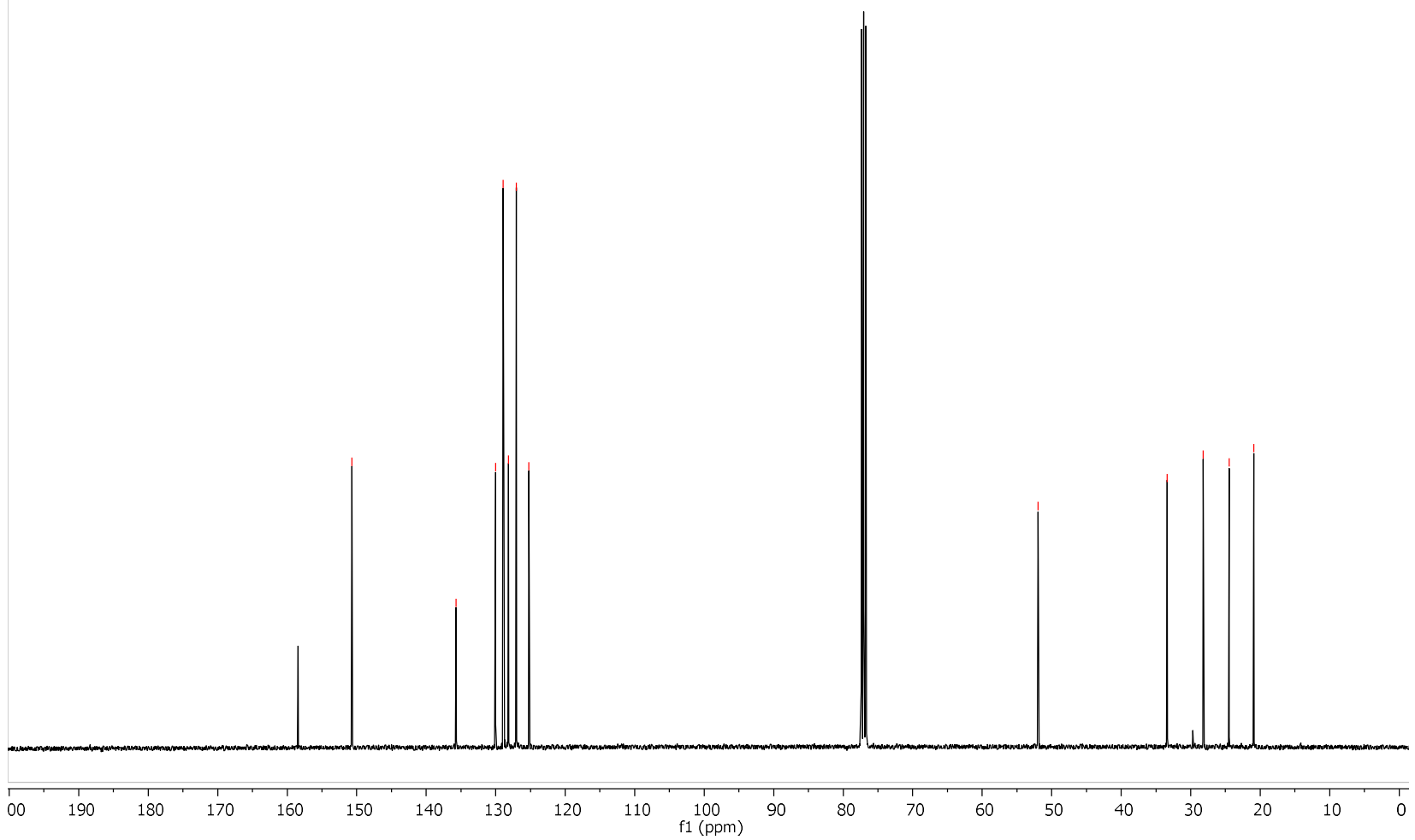


Phenyl(1-propyl-1H-1,2,4-triazol-5-yl)methanol (18e)
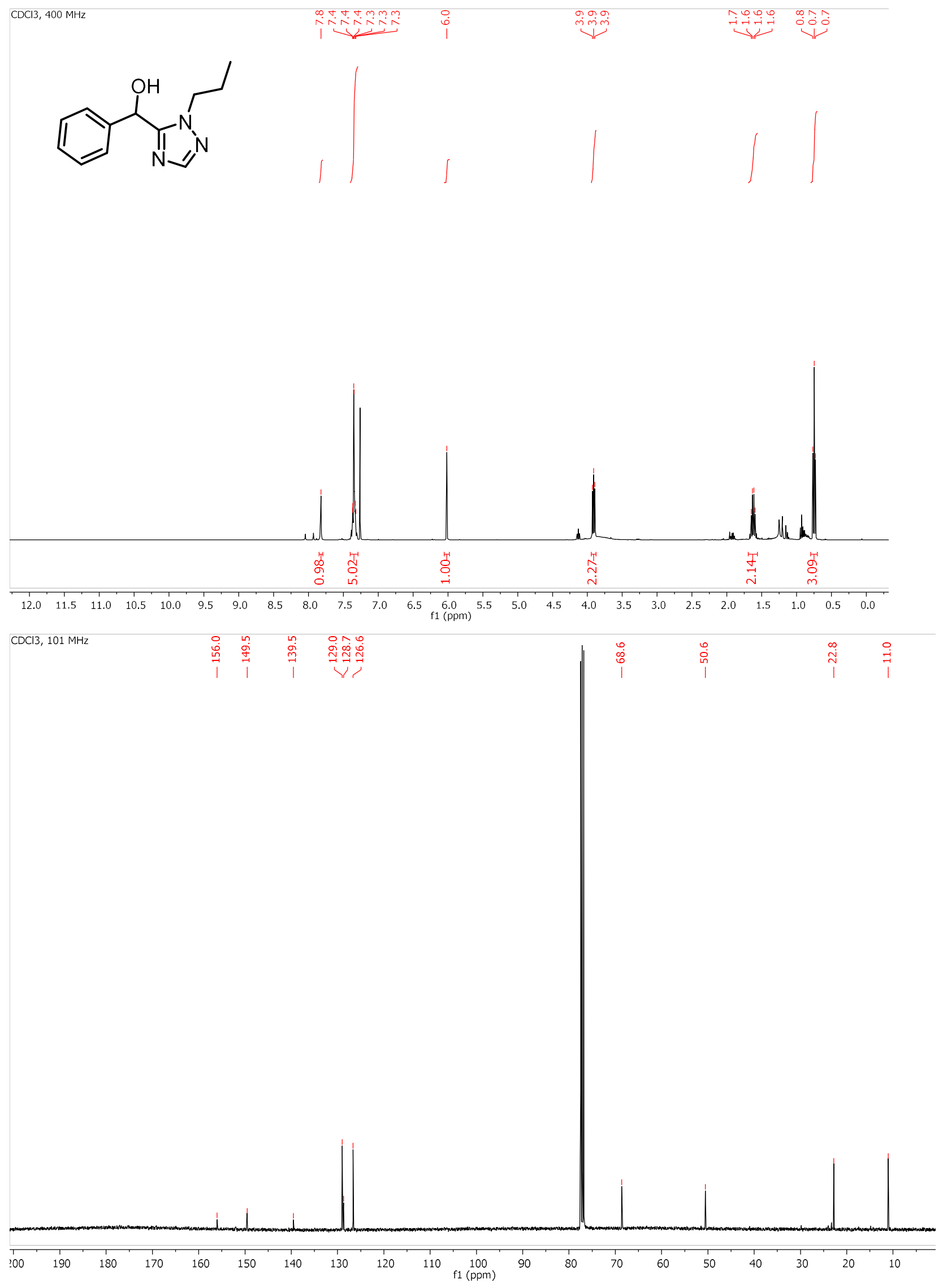

SI 64 
Phenyl(1-benzyl-1H-1,2,4-triazol-5-yl)methanol (18f)
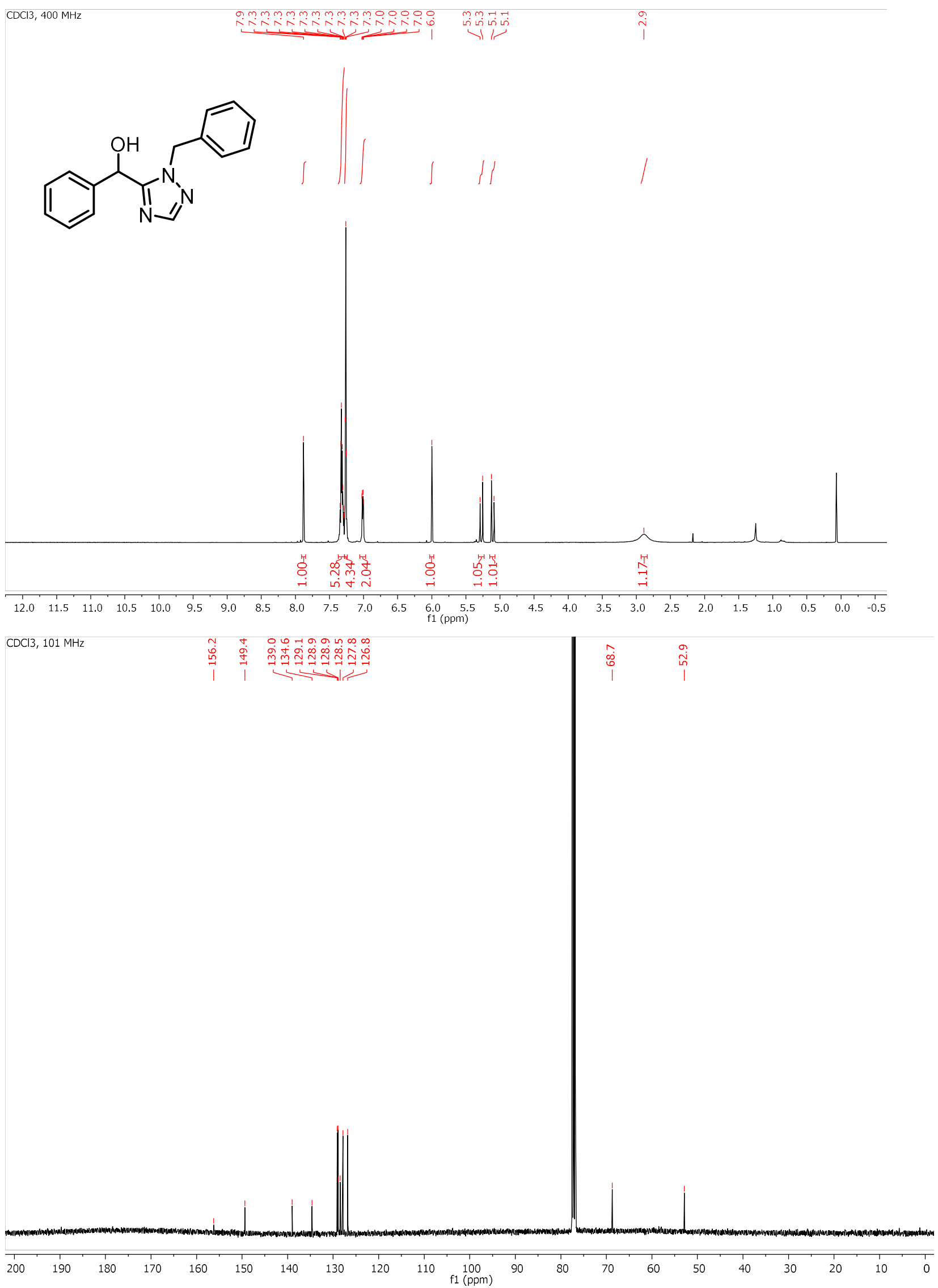
Phenyl(1-propyl-1H-1,2,4-triazol-5-yl)methanone (18g)
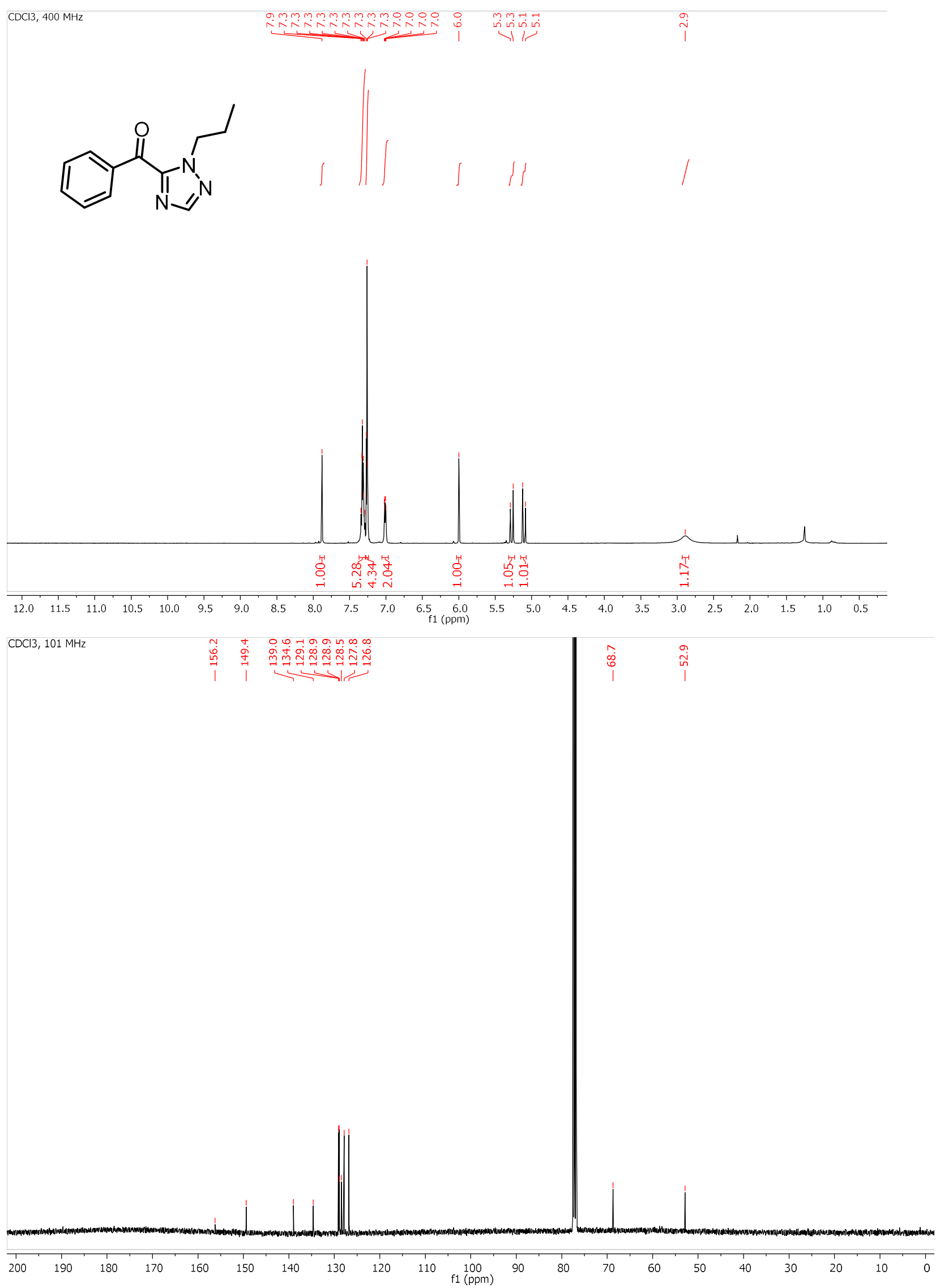

SI 66 

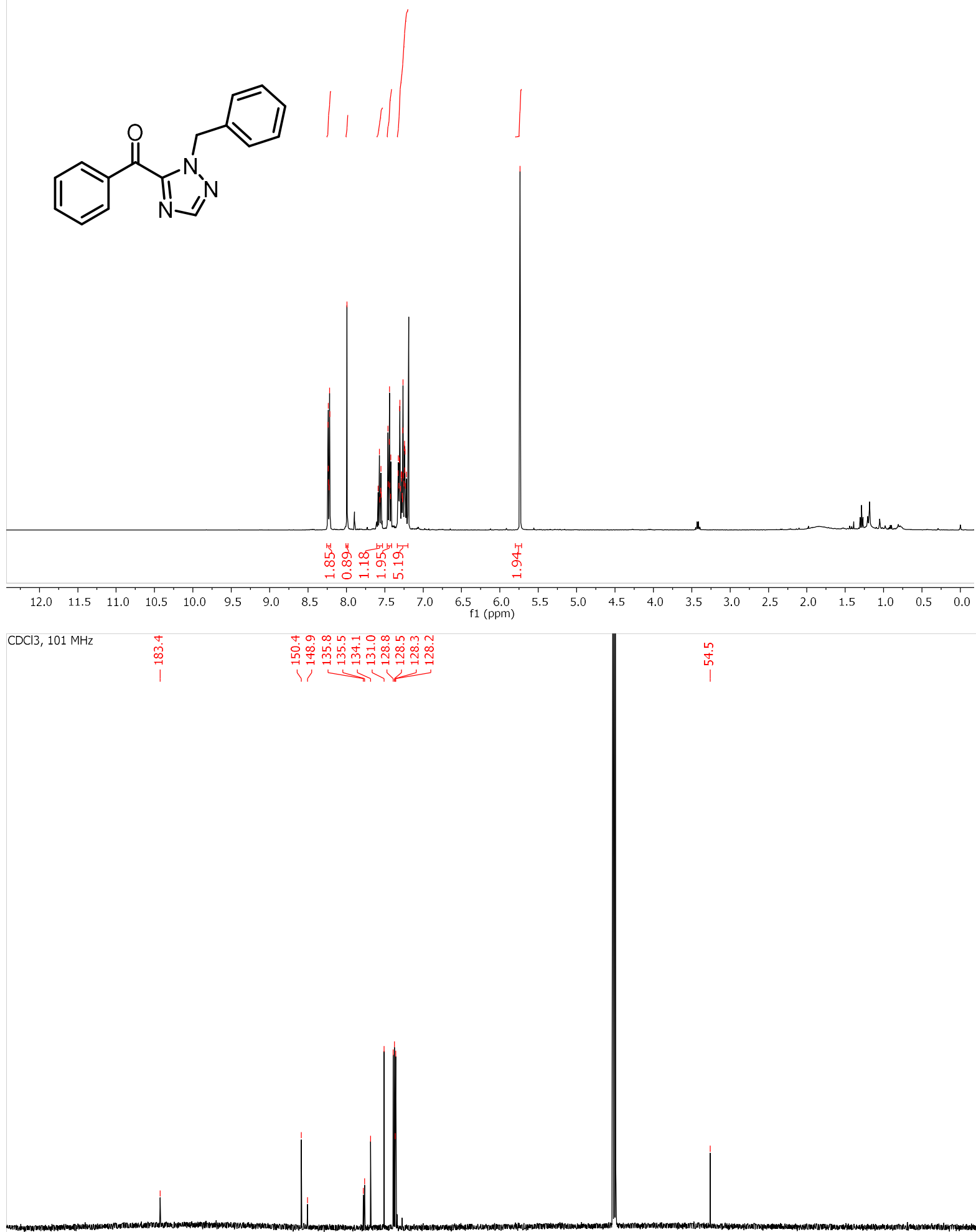
5-(4-Methoxyphenyl)-1-propyl-1H-1,2,4-triazole (18i)

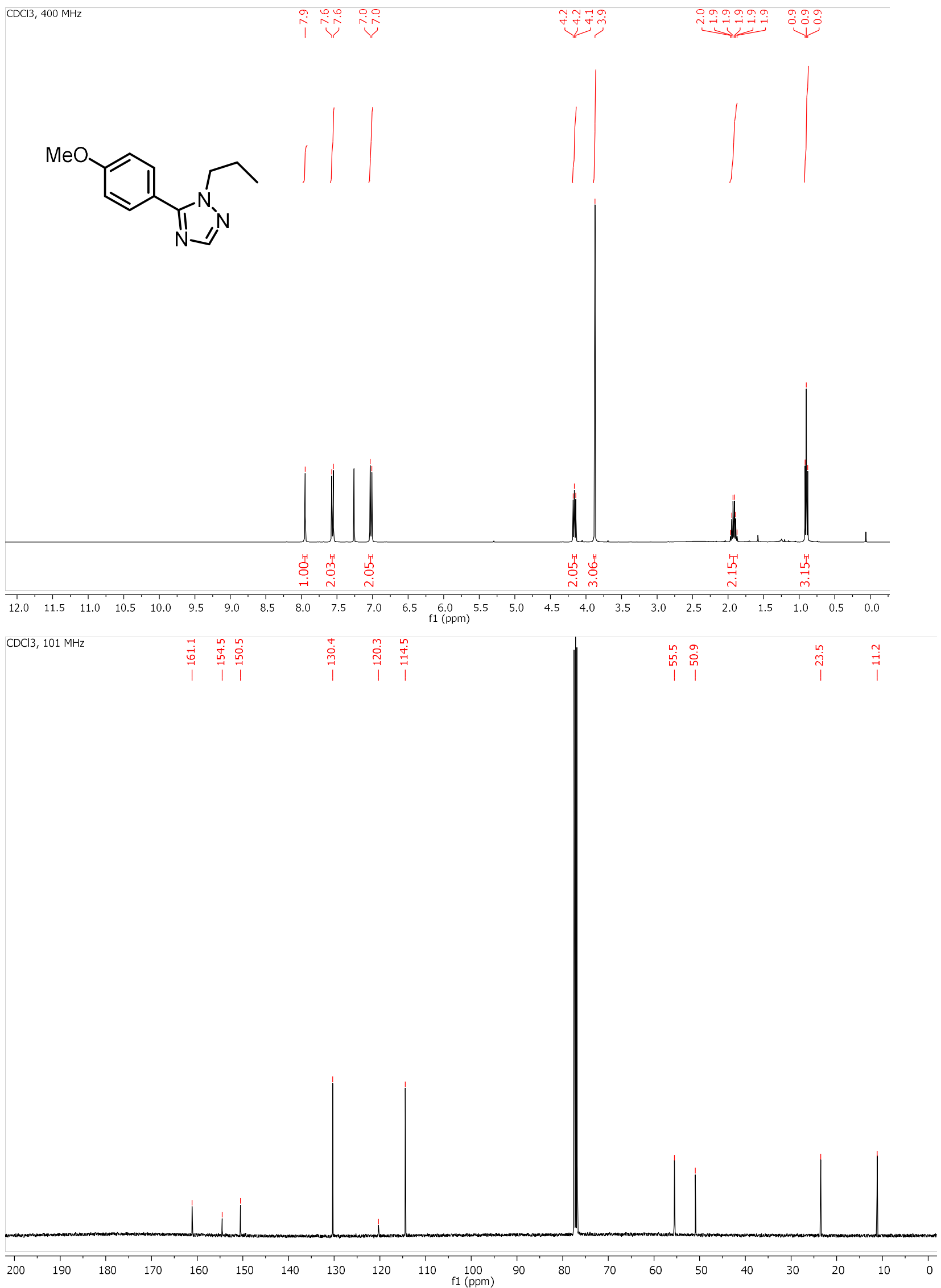


5-(4-Methoxyphenyl)-1-benzyl-1H-1,2,4-triazole (18j)

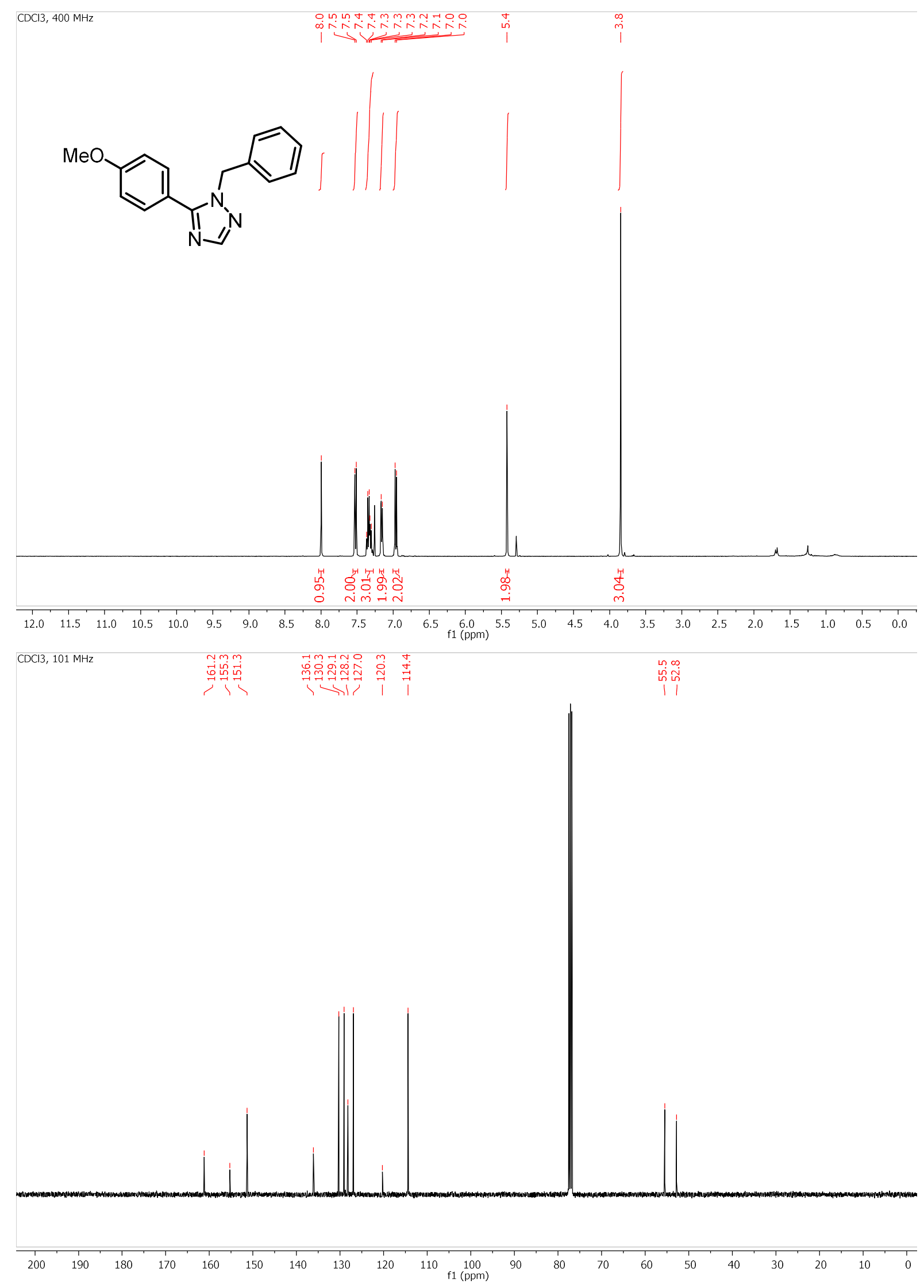

SI 69 
4-(1-Benzyl-1H-1,2,4-triazol-5-yl)benzonitrile (18k)<smiles>CCCn1ncnc1-c1ccc(C#N)cc1</smiles>
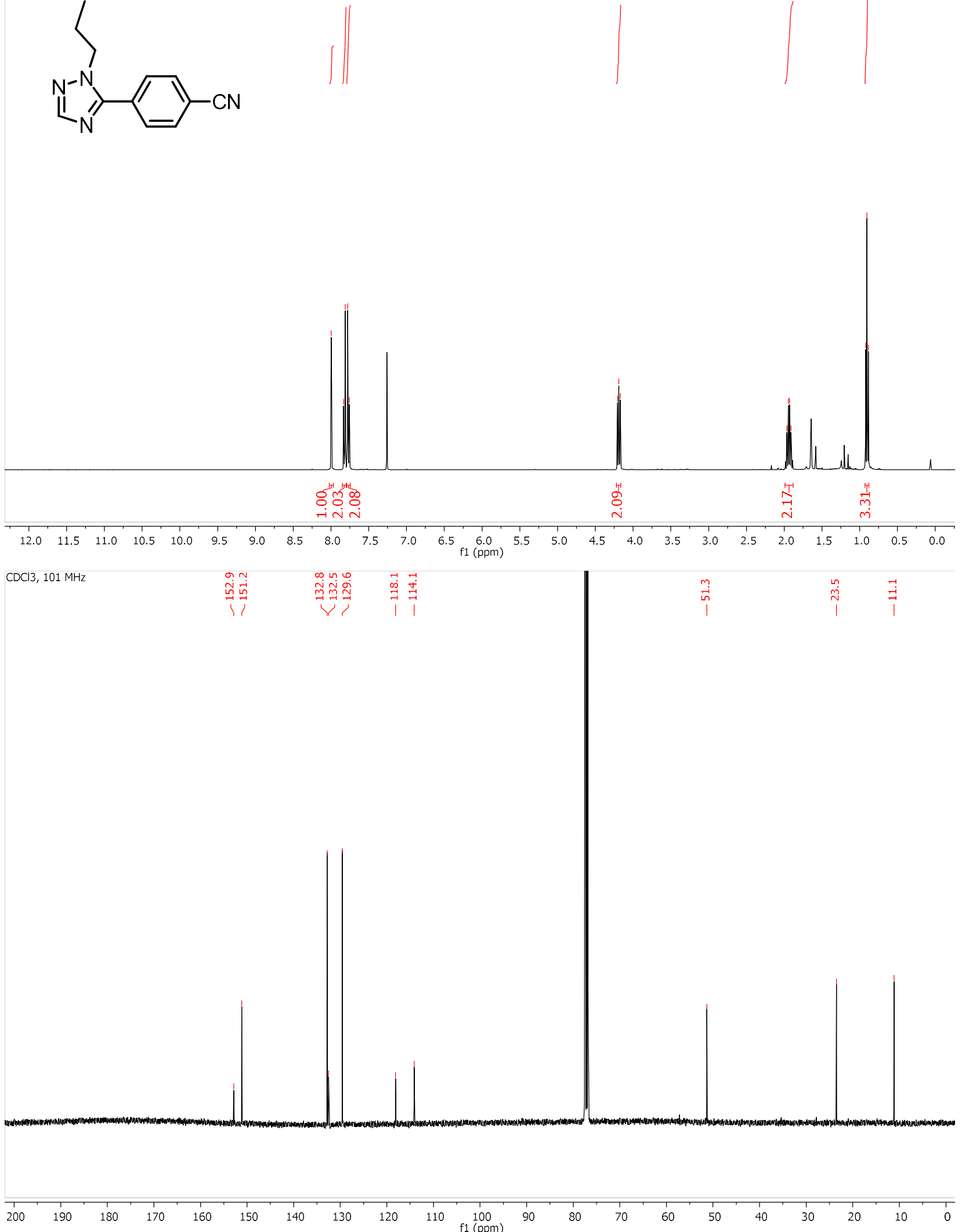
4-(1-Propyl-1 H-1,2,4-triazol-5-yl)morpholine (18I)
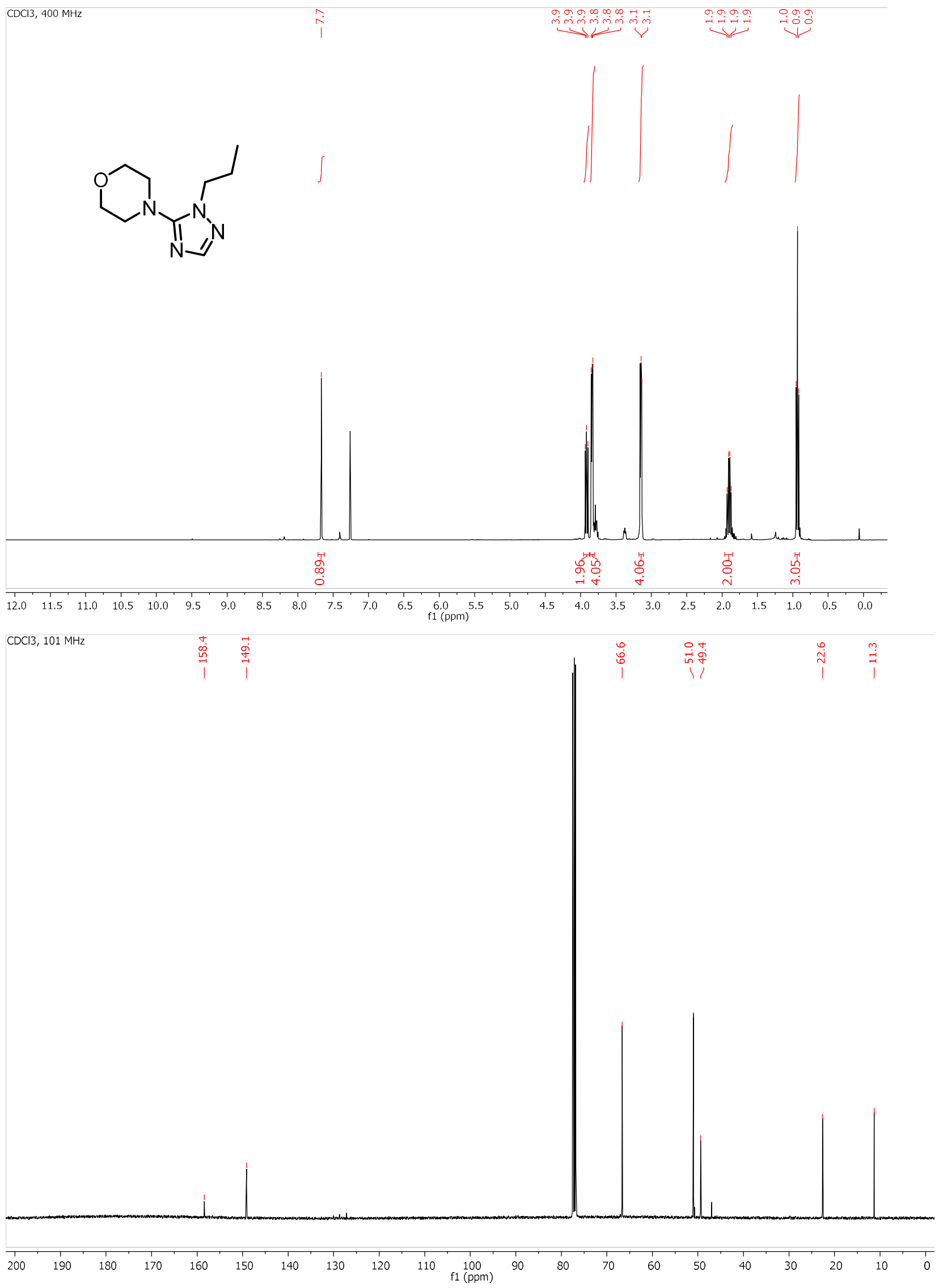
Ethyl 1-(1-benzyl-1H-1,2,4-triazol-5-yl)piperidine-3-carboxylate (18m)

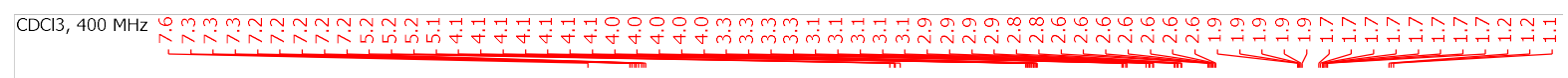<smiles>CCOC(=O)C1CCCN(c2ncnn2Cc2ccccc2)C1</smiles>
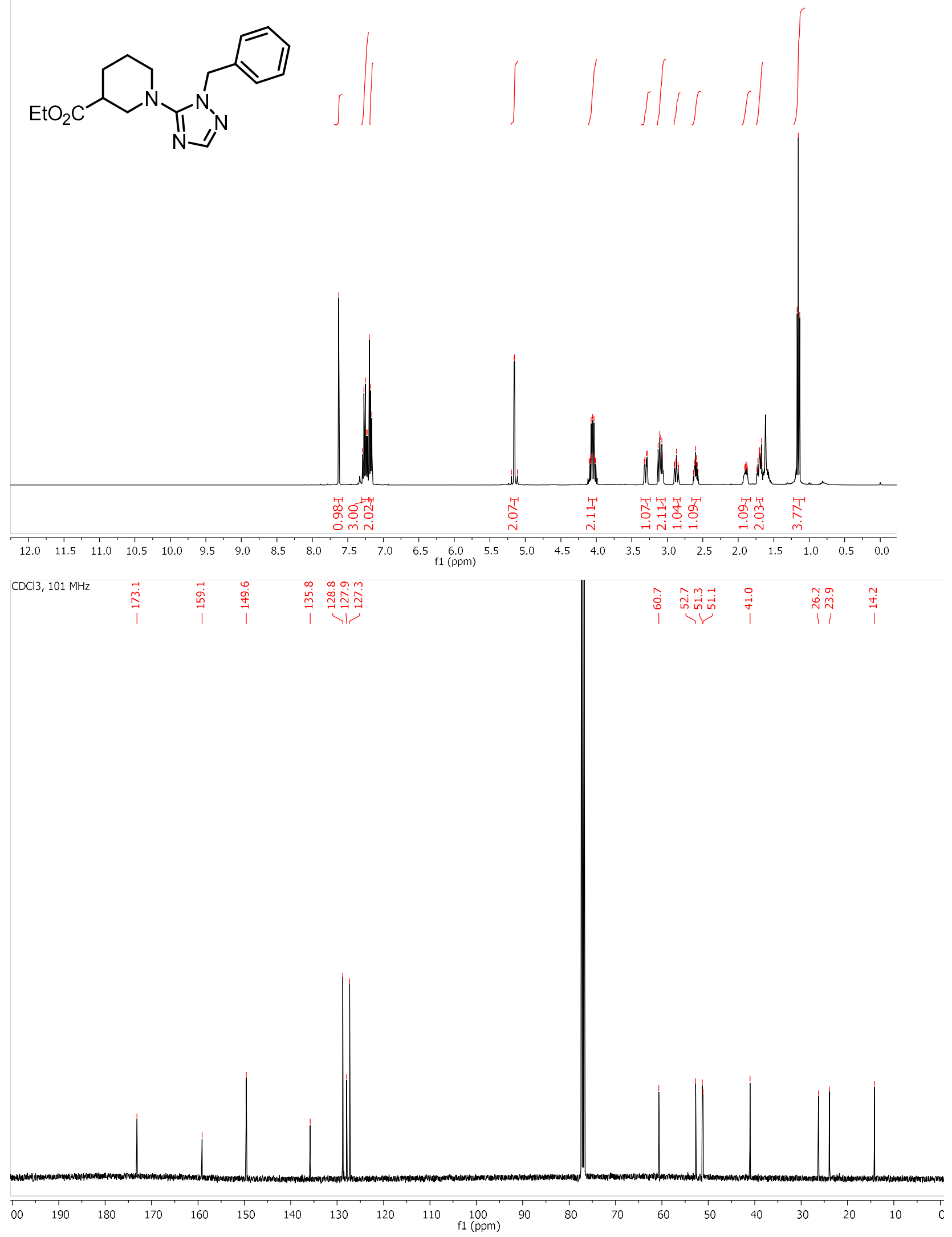

SI 72 
1-Benzyl-N-((1S,4S)-4-(3,4-dichlorophenyl)-1,2,3,4-tetrahydronaphthalen-1-yl)-Nmethyl-1H-1,2,4-triazol-5-amine (18n)
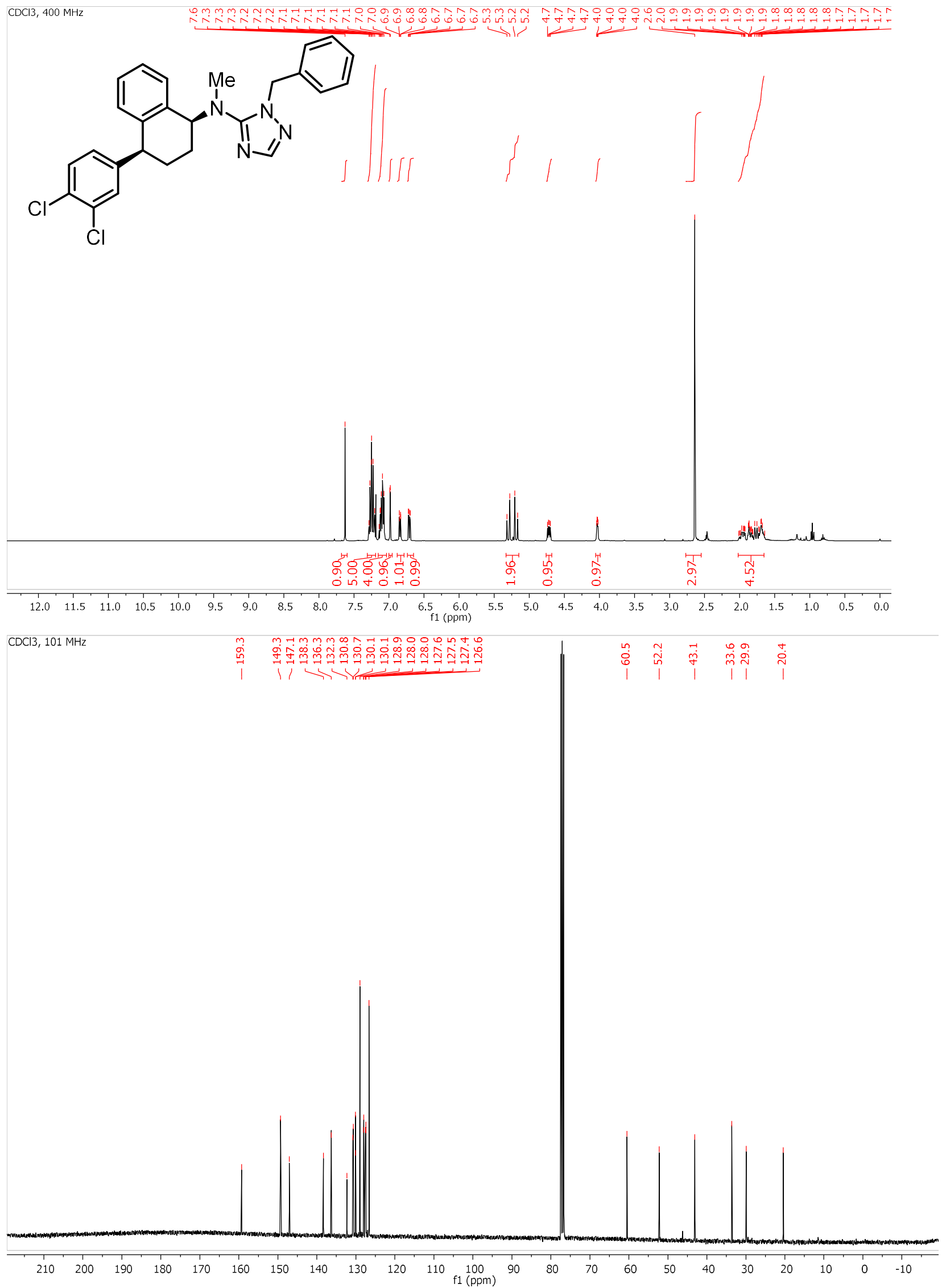
<smiles>CCCn1nc(C2C=CCCC2)nc1C1C=CCCC1</smiles>
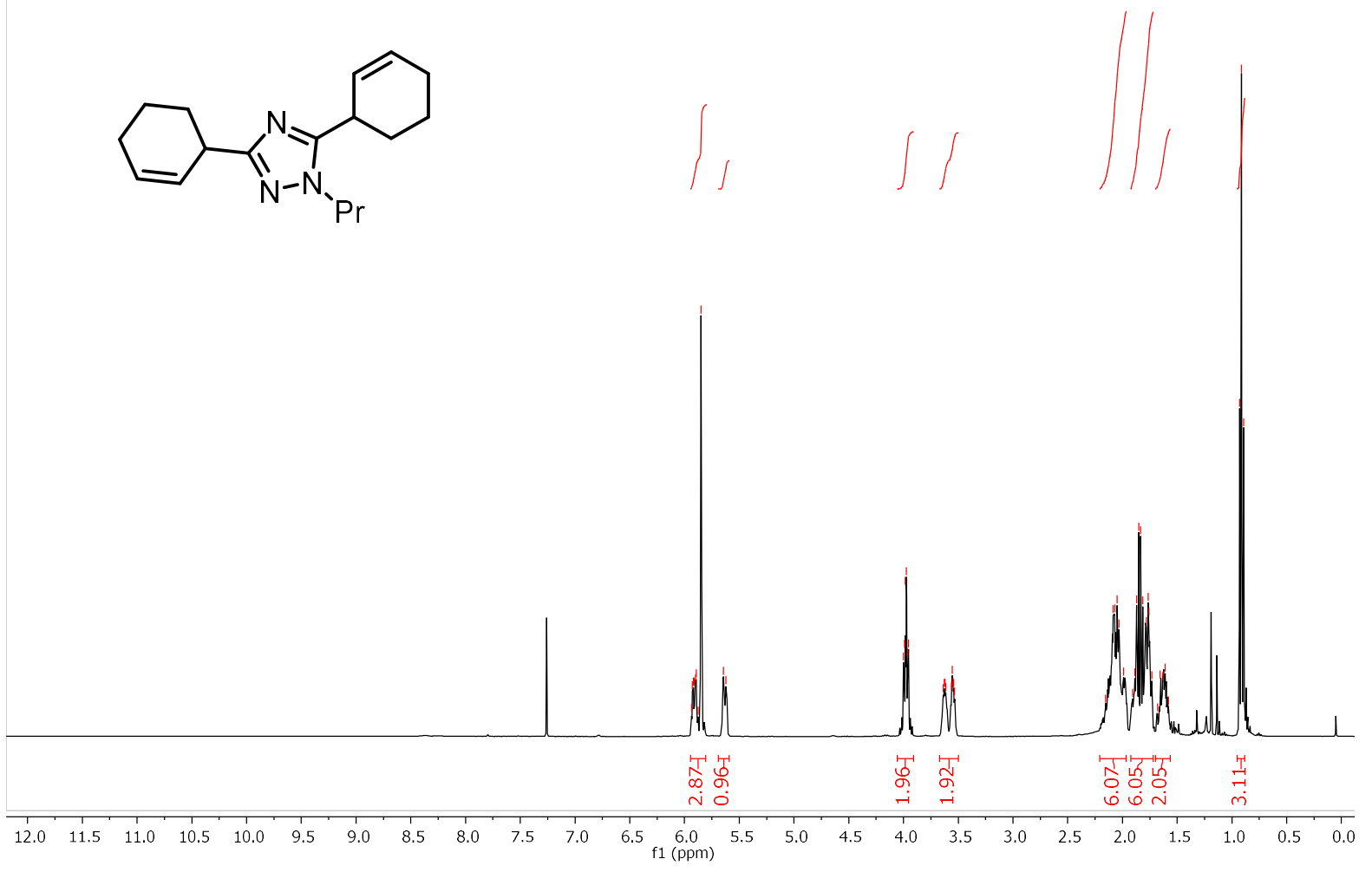

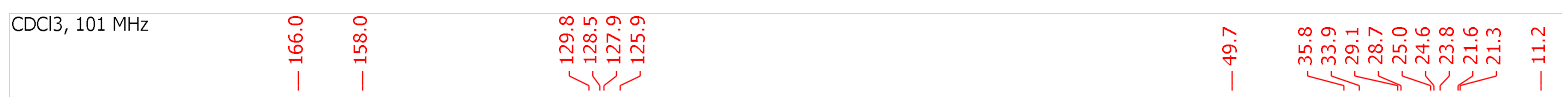

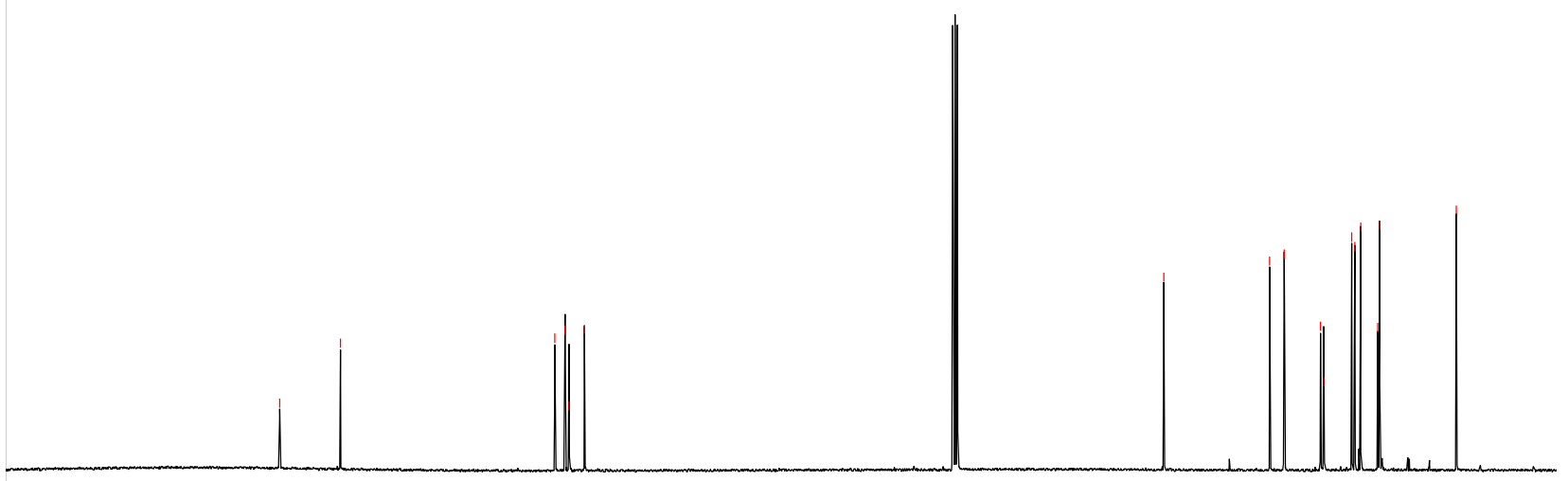

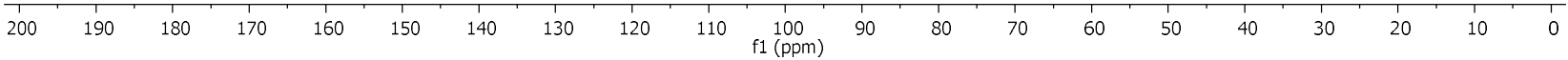


3,5-Bis(4-methoxyphenyl)-1-propyl-1H-1,2,4-triazole (19b)

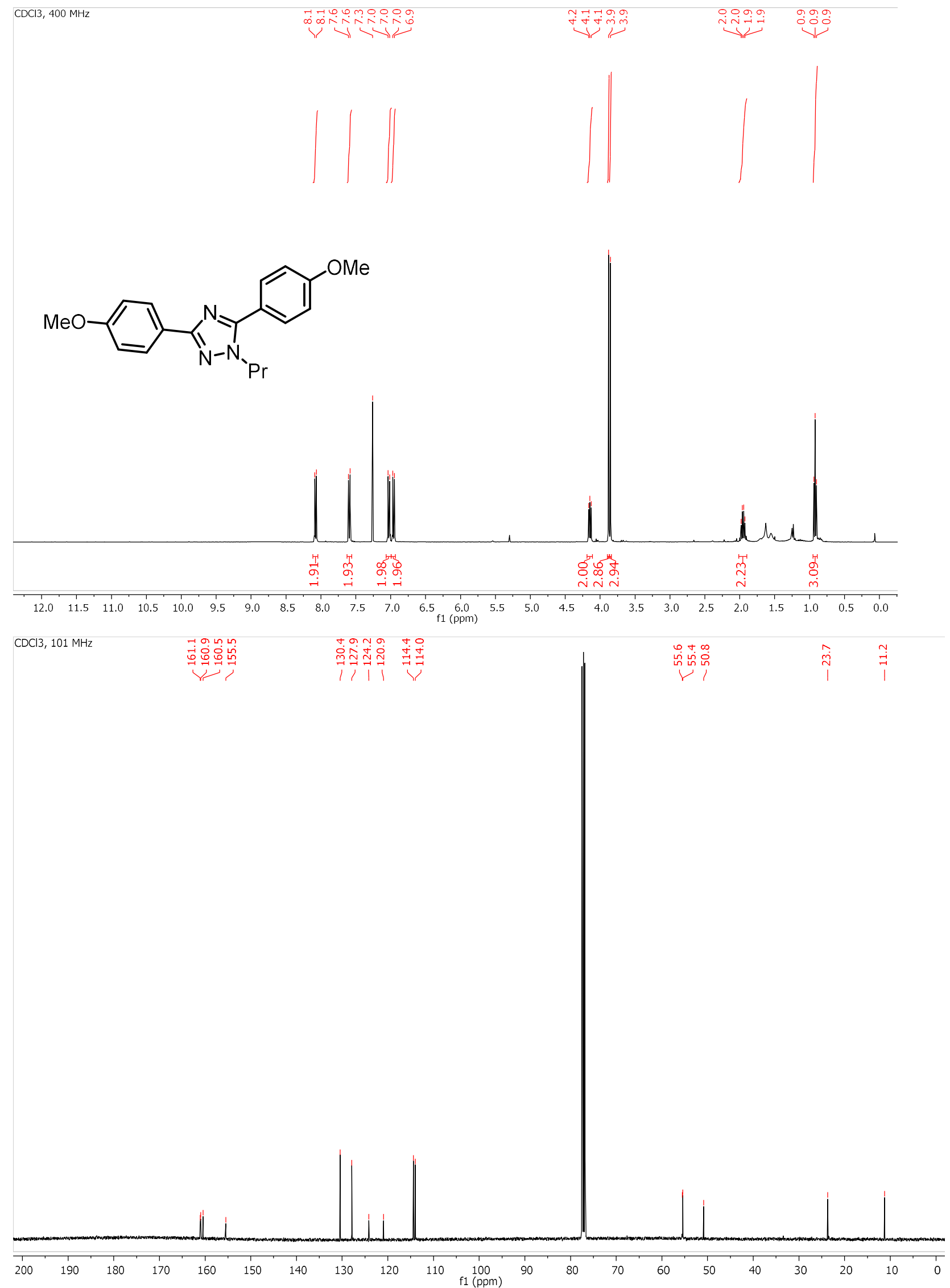


<smiles>CCCn1nc(C2C=CCCC2)nc1N1CCOCC1</smiles><smiles>CC1C=CC(C)C1</smiles>
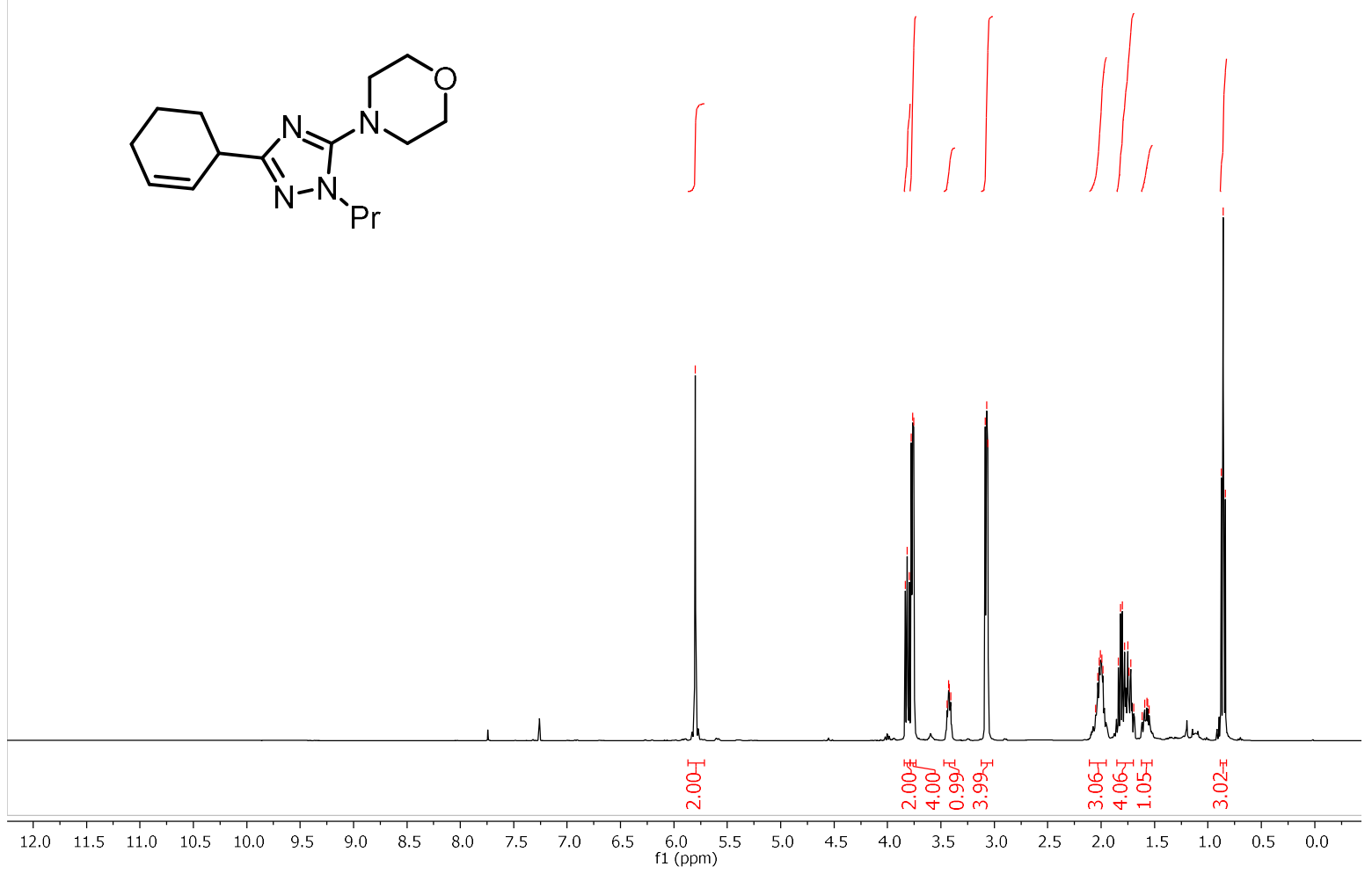
$\mathrm{CDCl} 3,101 \mathrm{MHz}$

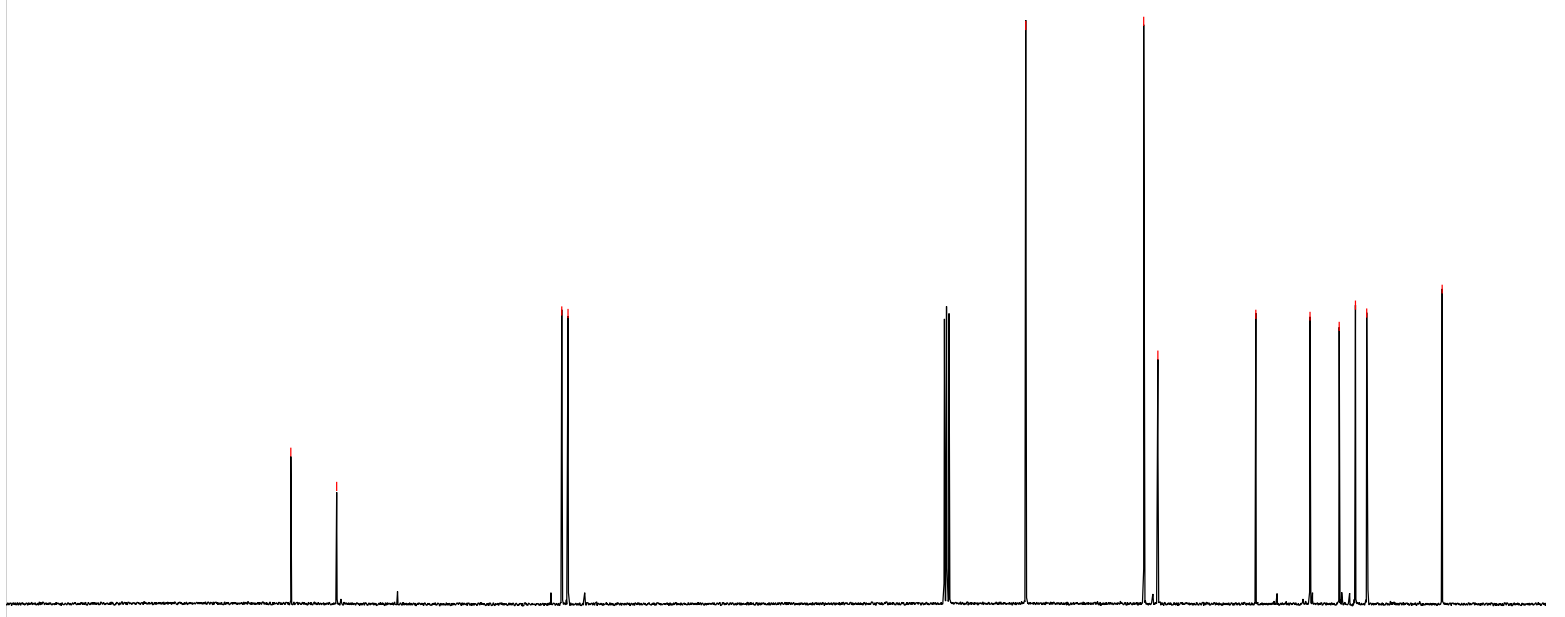


4-(5-(4-methoxyphenyl)-1-propyl-1H-1,2,4-triazol-3-yl)morpholine (19d)

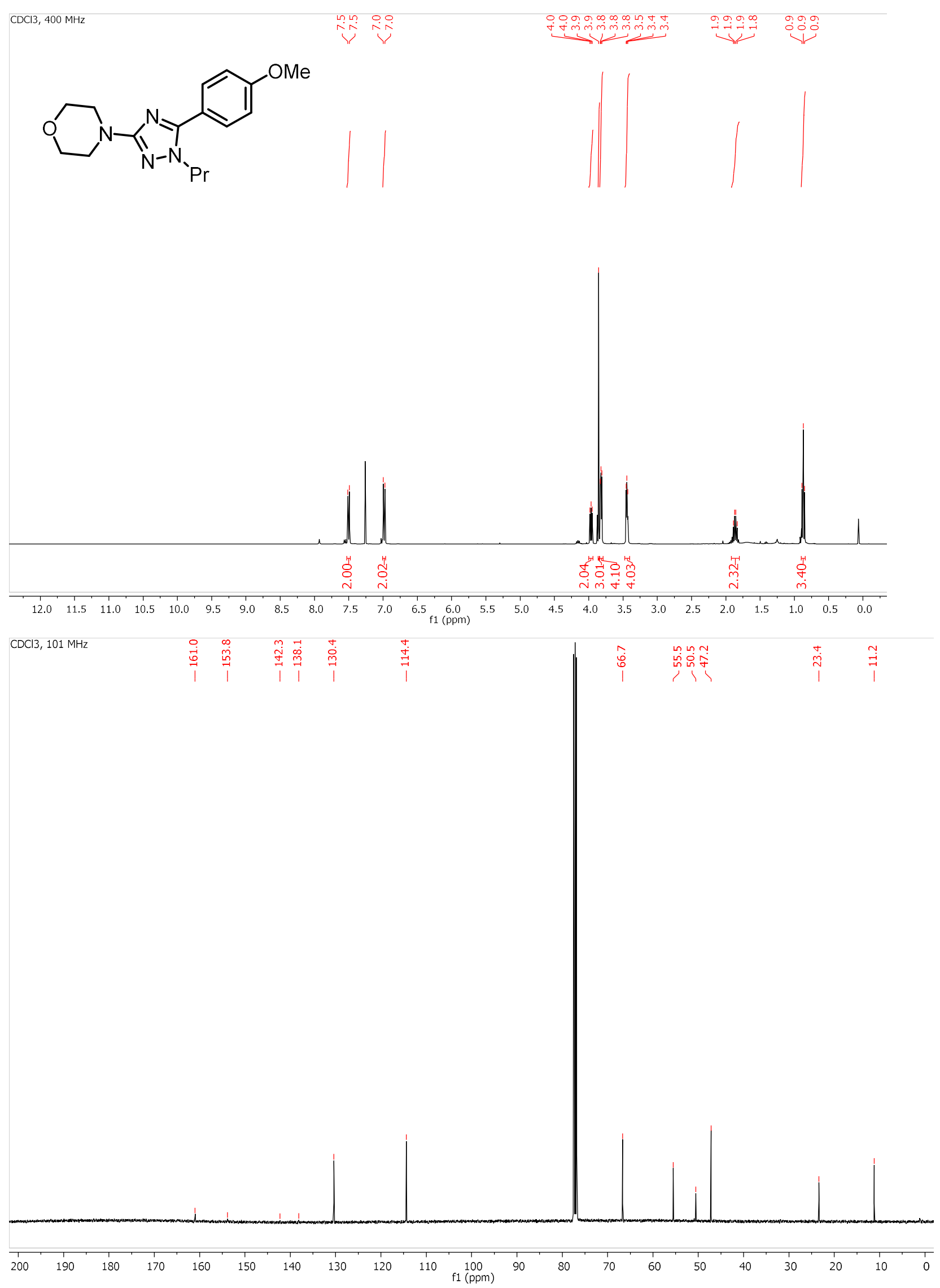

SI 77 
$5 H-[1,2,4]$ Triazolo[5,1-a]isoindole (20)

$\mathrm{CDCl} 3,400 \mathrm{MHz}$

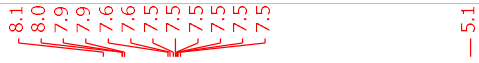<smiles>c1ccc2c(c1)Cn1ncnc1-2</smiles>
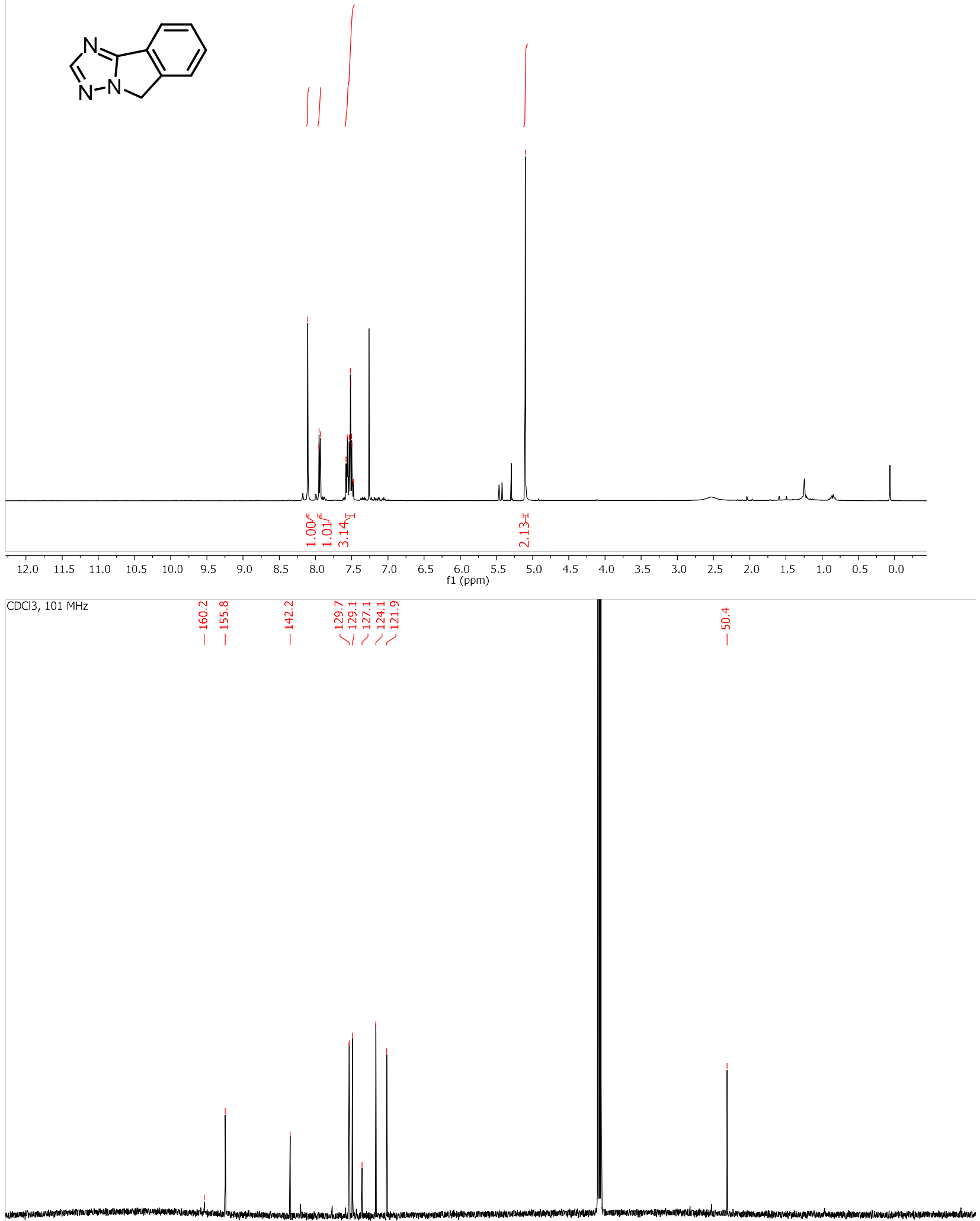

200

$\begin{array}{lllllll}100 & 190 & 180 & 170 & 160 & 150 & 140\end{array}$

$\begin{array}{lllllllll}80 & 70 & 60 & 50 & 40 & 30 & 20 & 10 & 0\end{array}$ 